\title{
Aerodynamic Characterization and Improved Testing Methods for the Space Launch System Liftoff and Transition Environment
}

\author{
David T. Chan*, John W. Paulson ${ }^{\dagger}$, Patrick R. Shea ${ }^{\ddagger}$, \\ Kenneth G. Toro ${ }^{\S}$, Peter A. Parker $₫$, Sean A. Commo $\|$ \\ NASA Langley Research Center, Hampton, VA, 23681
}

\begin{abstract}
Low-speed wind tunnel testing for the liftoff and transition environment of the Space Launch System (SLS) was recently completed in the NASA Langley Research Center 14by 22-Foot Subsonic Tunnel using $1.75 \%$-scale models of three SLS vehicle configurations. During the liftoff testing, the primary objective was to evaluate the aerodynamic forces and moments on the SLS launch vehicles, as the vehicle, launch tower, and mobile launch platform were subjected to ground winds from all directions at varying heights for the vehicle off the launch pad. Additionally, aerodynamic forces and moments were acquired for all three SLS vehicles during the transition phase from liftoff to ascent that covered a wide range of angles of attack and angles of sideslip. Details of the experimental setup including improved testing methods and a stiffer sting-balance system based on lessons learned from previous test entries are presented. Also, a new force measurement technique was applied during the test where two subminiature six-component load cells were installed in each Solid Rocket Booster (SRB) to acquire forces and moments for each SRB separately from the full vehicle forces and moments measured by the main strain gauge balance. Finally, sample results from the experiment are presented including improved overall data coverage, evaluation of the new SRB data, and smoke flow visualization photos.
\end{abstract}

\section{Nomenclature}

\begin{tabular}{|c|c|c|c|}
\hline Symbols & & $R e$ & Freestream unit Reynolds Number, \\
\hline$C A$ & Axial force coefficient, body axis & & $10^{6} / f t$ \\
\hline$C L L$ & Rolling moment coefficient, body axis & $V_{\infty}$ & Freestream velocity, ft/sec \\
\hline$C L M$ & $\begin{array}{l}\text { Pitching moment coefficient, body } \\
\text { axis }\end{array}$ & $\begin{array}{l}\alpha \\
\beta\end{array}$ & $\begin{array}{l}\text { Body axis angle of attack, deg } \\
\text { Body axis angle of sideslip, deg }\end{array}$ \\
\hline$C L N$ & Yawing moment coefficient, body & $\begin{array}{l}\alpha_{p} \\
\phi_{p}\end{array}$ & $\begin{array}{l}\text { Missile axis angle of attack, deg } \\
\text { Missile axis roll angle, deg }\end{array}$ \\
\hline$C N$ & Normal force coefficient, body axis & $\psi_{a z m}$ & Wind azimuth angle, deg \\
\hline$C Y$ & Side force coefficient, body axis & Subscripts & \\
\hline$h$ & $\begin{array}{l}\text { Height of the vehicle off the launch } \\
\text { pad, ft }\end{array}$ & $p$ & Missile axis \\
\hline$h / L$ & $\begin{array}{l}\text { Nondimensional launch height } \\
\text { parameter }\end{array}$ & $\begin{array}{l}a z m \\
S R B\end{array}$ & $\begin{array}{l}\text { Wind azimuth } \\
\text { SRB-related quantity }\end{array}$ \\
\hline$L$ & Height of the launch tower, $\mathrm{ft}$ & Units & \\
\hline$M_{\infty}$ & Freestream Mach number & ${ }^{\circ}, \operatorname{deg}$ & degrees \\
\hline$q_{\infty}$ & Freestream dynamic pressure, psf & $\mathrm{ft}$ & feet \\
\hline $\begin{array}{l}\text { *Researc } \\
{ }^{\dagger} \text { Researc } \\
{ }^{\ddagger} \text { Researc } \\
\text { § Researc } \\
\text { I Team L } \\
\text { " Balance }\end{array}$ & $\begin{array}{l}\text { ospace Engineer. Configuration Aerodynamic } \\
\text { ospace Engineer. Analytical Mechanics Assoc } \\
\text { ospace Engineer. Configuration Aerodynamic } \\
\text { gineer. System Engineering \& Engineering Me } \\
\text { Advanced Measurement \& Data Systems Bra } \\
\text { ineer. Advanced Measurement \& Data Systen }\end{array}$ & $\begin{array}{l}\text { Branch. Senior } \\
\text { ates, Inc. Associ } \\
\text { Branch. Membe } \\
\text { hods Branch. M } \\
\text { h. peter.a.parke } \\
\text { Branch. sean.a }\end{array}$ & $\begin{array}{l}\text { Member AIAA. david.t.chan@nasa.gov } \\
\text { te Fellow AIAA.john.w.paulson@nasa.gov } \\
\text { AIAA.patrick.r.shea@nasa.gov } \\
\text { mber AIAA.kenneth.g.toro@nasa.gov } \\
\text { @nasa.gov } \\
\text { commo@nasa.gov }\end{array}$ \\
\hline & & & \\
\hline
\end{tabular}




\begin{tabular}{|c|c|c|c|}
\hline $\mathrm{ft} / \mathrm{sec}$ & feet per second & & Regulations \\
\hline psf & pounds per square foot & LAS & Launch Abort System \\
\hline & & LOT & Liftoff and Transition \\
\hline Acronyms & & LOX & Liquid Oxygen \\
\hline $14 \times 22$ & 14-by 22 -Foot Subsonic Tunnel & LSRB & Left (Port) Solid Rocket Booster \\
\hline ATI-IA & ATI Industrial Automation & MLP & Mobile Launch Platform \\
\hline $\mathrm{BMC}$ & Balance Moment Center & $\mathrm{MPCV}$ & Multi-Purpose Crew Vehicle \\
\hline BSM & Booster Separation Motor & MRP & Moment Reference Point \\
\hline CFD & Computational Fluid Dynamics & NASA & National Aeronautics and Space \\
\hline CG & Center of Gravity & & Administration \\
\hline CSE & Core Stage Engine & RSRB & Right (Starboard) Solid Rocket \\
\hline ESP & Electronically Scanned Pressure & & Booster \\
\hline $\mathrm{F} \& \mathrm{M}$ & Force \& Moment & SBU & Sensitive But Unclassified \\
\hline FMS & Force Measurement System & SLS & Space Launch System \\
\hline GN\&C & Guidance, Navigation, and Control & SRB & Solid Rocket Booster \\
\hline GOX & Gaseous Oxygen & TSMU & Tail Service Mast Umbilical \\
\hline ITAR & International Traffic in Arms & WT & Wind Tunnel \\
\hline
\end{tabular}

\section{Introduction}

7 He Space Launch System (SLS) ${ }^{1}$ is the National Aeronautics and Space Administration's (NASA) advanced heavy-lift launch vehicle that will enable human exploration beyond Earth's orbit for the United States. The SLS is designed to launch astronaut crews aboard the Orion Multi-Purpose Crew Vehicle on missions to explore multiple, deep-space destinations. The launch vehicle consists of a central core stage that is powered by four RS-25 liquid propellent core stage engines (CSE) and by two five-segment solid rocket boosters (SRB) attached to each side of the core stage. The SLS design will continue to evolve into configurations with increasingly higher lift capacity and the capability for both crew and cargo missions as shown in Figure 1. The aerodynamic characterization of the initial Block 1 configuration and the evolved Block 1B configurations has been the focus of the SLS program in recent years.

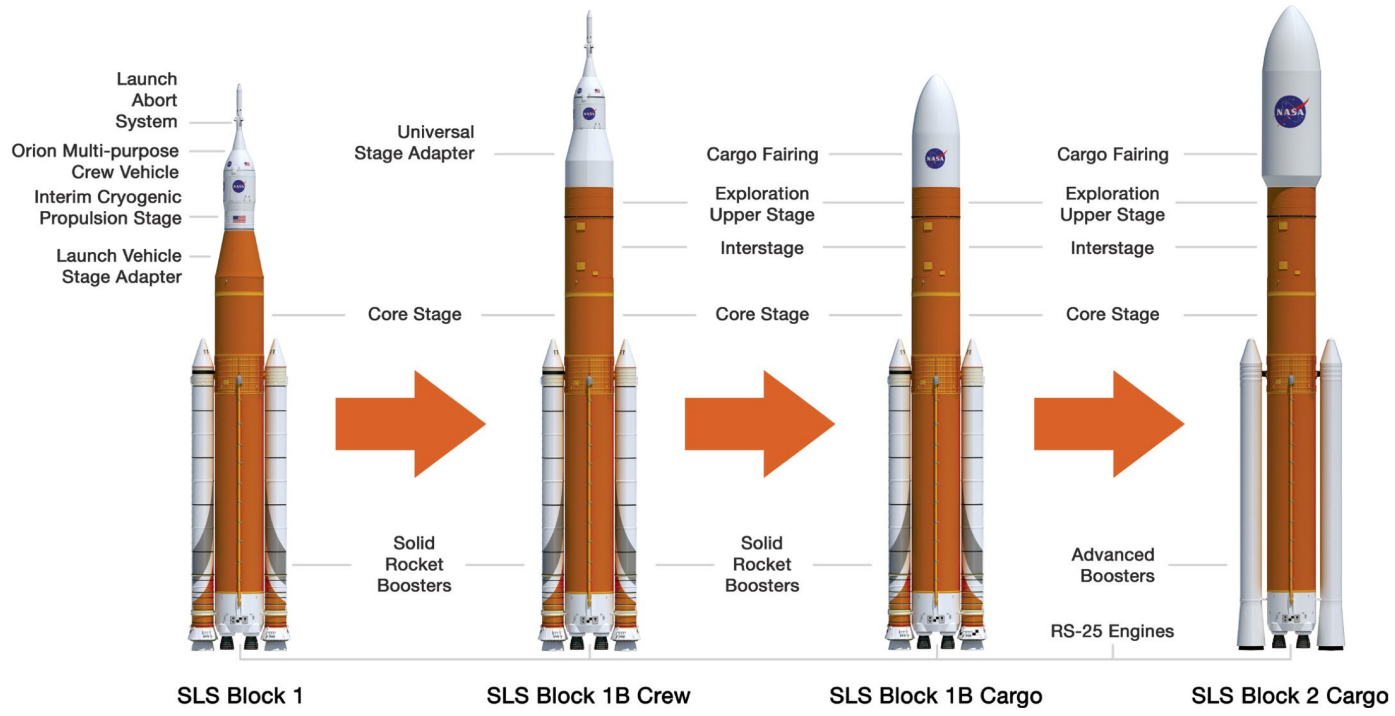

Figure 1. Space Launch System planned configurations for crew and cargo missions. ${ }^{1}$

The liftoff and transition (LOT) phase of flight includes the liftoff of the SLS launch vehicle from the mobile launch platform (MLP) and the vehicle's transition into forward flight during the ascent phase. During the LOT phase, the vehicle, launch tower, and MLP are subjected to ground winds at the launch complex and these ground winds can have an effect on the clearance distance between the vehicle and the launch tower during liftoff. To characterize the aerodynamics of the vehicle during liftoff and transition, 
data need to be obtained in the incompressible or low-subsonic flow regime (Mach $\leq 0.3$ ). As part of flight simulations performed by the Guidance, Navigation, \& Control (GN\&C) group within the SLS program, the aerodynamic data gathered from LOT ground experiments are used to ensure that the vehicle will not enter a keep-out zone around the launch tower during its liftoff from the launch pad. The aerodynamic data are also used once the vehicle has cleared the launch tower and begins its ascent through the atmosphere.

There have been two other LOT experiments performed for NASAdeveloped launch vehicles, one for the Ares I launch vehicle from the Constellation program described by Capone et al. in Ref. 2 and one for early configurations of the SLS launch vehicle. Both experiments were performed in the 14-by 22-Foot Subsonic Tunnel located at the NASA Langley Research Center in Hampton, Virginia. The first SLS LOT test entry, designated Test 609, was conducted in July-August 2013 and described by Pinier et al. in Ref. 3. This paper describes the second $1.75 \%$ scale SLS LOT wind tunnel test entry, designated Test 633 and conducted in March-May 2017, where updated and evolved SLS launch vehicle configurations were tested and several testing method improvements were implemented based on lessons learned from Test 609. A photo of the SLS LOT model from Test 633 is shown in Figure 2. The aerodynamic data from Test 633 were also used to update the current version of the SLS liftoff and transition aerodynamic database.

This paper will present an overview of the vehicle and launch tower test articles and the wind tunnel facility, then describe the experimental setup with an emphasis on the improvements made to the sting-balance system and the implementation of a new force measurement capability for the SRBs. Sample aerodynamic data results will be presented, but since the SLS program data are under Sensitive But Unclassified (SBU)

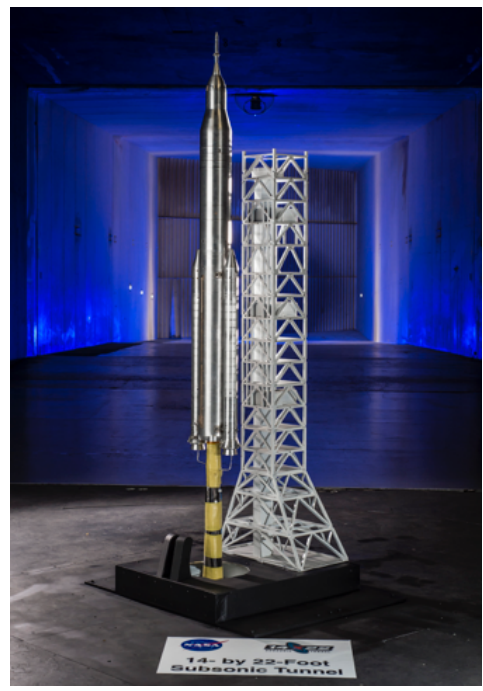

Figure 2. Photo of SLS Liftoff and Transition model in the 14by 22-Foot Subsonic Tunnel. and International Traffic in Arms Regulations (ITAR) restrictions, the data plots will not show absolute values of the aerodynamic coefficients. Finally, sample results from smoke flow visualization sessions will be presented with some interesting and surprising flow features.

\section{Wind Tunnel Description}

The 14-by 22-Foot Subsonic Tunnel $(14 \times 22)^{4}$ located at the NASA Langley Research Center is an atmospheric, closed circuit, low-speed wind tunnel that is used to assess aerodynamic performance of powered and unpowered models of various civil and military aircraft, as well as launch vehicles and spacecraft. The 40-foot diameter fan powered by a 12,000 horsepower drive motor allows continuous control of Mach number in the test section from 0 to 0.3 . Figure 3 (a) shows an aerial view of the tunnel.

The facility has several model carts that can be used depending on the type of testing required and the type of model support required. Model Cart \#2 has a vertical post mounting system that was originally designed for ground effect testing of fighter and transport aircraft models. It has a pitch range of $-10^{\circ}$ to $+50^{\circ}$ and vertical motion range from the floor of the test section to about the test section centerline. With this range of motion, the model can be kept at or near the test section centerline for the entire pitch range. The floor turntable on Cart \#2 also allows the entire model support system to be rotated nearly an entire revolution $\left(330^{\circ}\right)$ to position the model at different yaw orientations to the oncoming flow. For the SLS experiment described in this paper, Cart \#2 was positioned in the forward location in the test section with an empty model cart positioned in the aft location as shown in Figure 3(b-c).

The side walls and ceiling of the test section can be removed in order to minimize wall interference when testing large models or rotorcraft at low speeds. However, for the SLS experiment described in this paper, the test section was closed due to the relatively small size and blockage ratio of the launch tower and SLS model. The facility is also equipped with a boundary layer removal system that can reduce the thickness of the boundary layer on the floor of the test section. The thickness of the boundary layer in the test section near the location of the SLS model is about 5 inches. Previous LOT experiments performed by Capone et al. ${ }^{2}$ and Pinier et al. ${ }^{3}$ did not use the boundary layer removal system because the wind tunnel model was always above this height from the floor and they concluded that the floor boundary layer effects were minimal and could be ignored. Similarly, the system was not used for SLS LOT Test 633. 


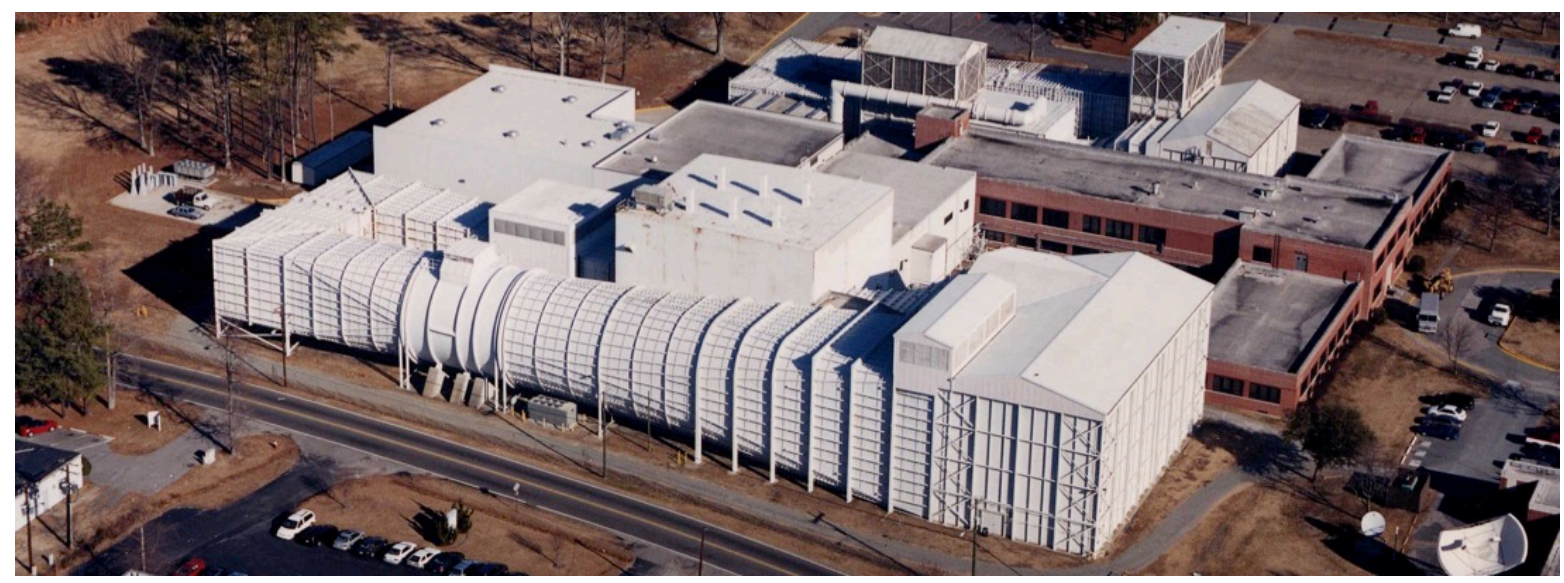

(a) Aerial view

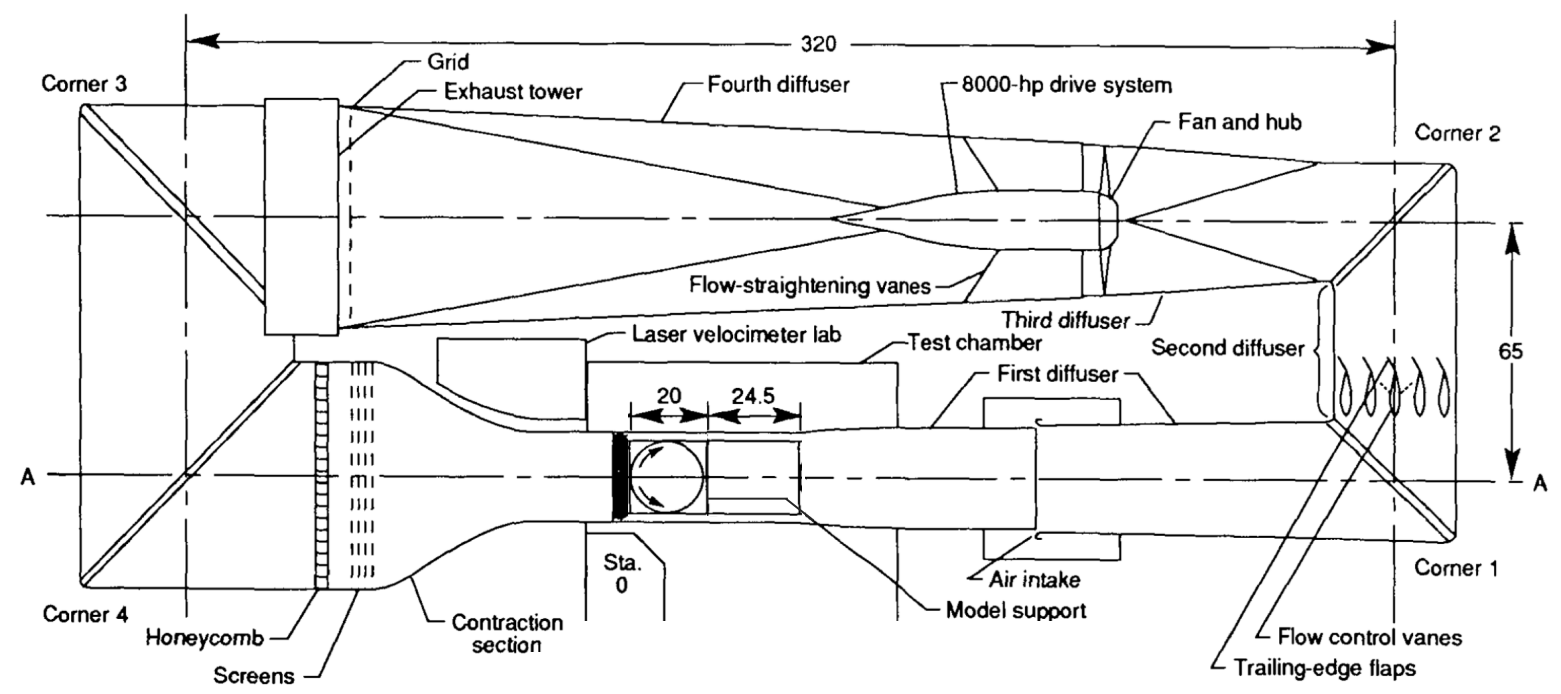

(b) Schematic of tunnel circuit

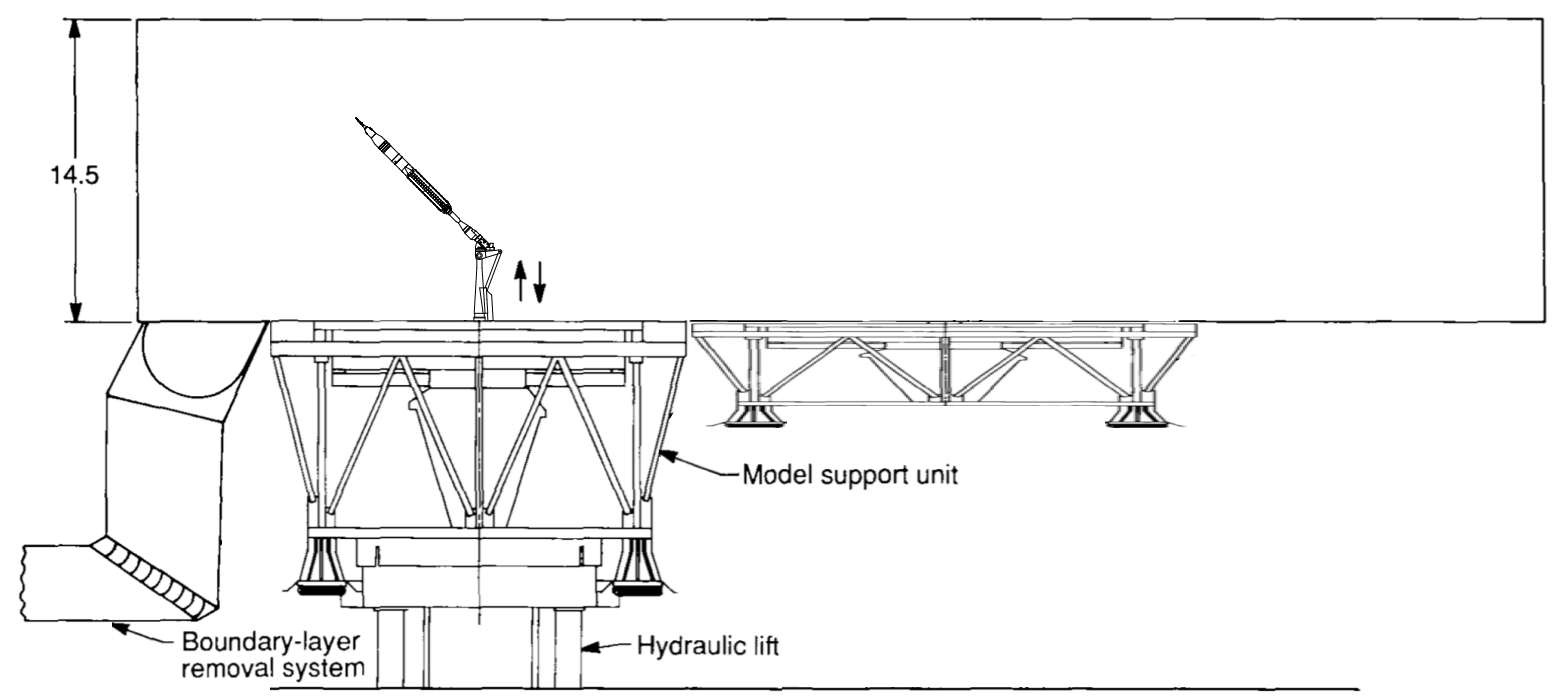

(c) Side view of test section with the SLS model

Figure 3. The 14-by 22-Foot Subsonic Tunnel at the NASA Langley Research Center shown with an (a) aerial view, (b) schematic of tunnel circuit, and (c) side view of test section. Linear dimensions are given in feet. 


\section{Test Article Description}

\section{A. SLS Vehicles}

Three $1.75 \%$-scale SLS vehicle configurations were tested during Test 633 at the $14 \times 22$. The three configurations were the Block 1 crew configuration designated SLS-10008, the Block 1B crew configuration designated SLS-28005, and the Block 1B cargo configuration designated SLS-27005. The test articles were designed to have a common lower core stage with SRBs and separate upper stages and noses for the three configurations, as shown in Figure 4. This allowed for simple model changes when going from one SLS configuration to the other. Many of the larger protuberances on the vehicles were included in the models and they were bonded in place and were not removed during the course of testing. The models were fabricated mostly out of aluminum, with the protuberances 3D-printed out of cobalt-chromium. Also, the existing SRB attach brackets from Test 609 were replaced in Test 633 by new 3D-printed brackets. These new brackets and some modifications to the existing SRB bodies were required to accommodate the new SRB force measurement system that will be described in a later section.

$1.75 \%$-scale Models

- Common core stage and SRBs

- Separate upper stages

- Mostly made out of aluminum

- Some 3D-printed parts

\begin{tabular}{|c|c|}
\hline Vehicle Element & Protuberances \\
\hline Core Stage & Systems Tunnel (1) \\
LOX Feedline (2) \\
\hline \multirow{4}{*}{ LAS Tower (1) } \\
& LAS Nozzer Stage (4) \\
& LAS Systems Tunnel (1) \\
& MPCV Umbilical (1) \\
& Upper Stage Systems Tunnel (1) \\
& Cameras (4) \\
& GOX Vent Cover (1) \\
\hline \multirow{3}{*}{ 27005 Upper Stage } & Upper Stage Systems Tunnel (1) \\
& Cameras (4) \\
& GOX Vent Cover (1) \\
\hline & LAS Tower (1) \\
& LAS Nozzles (4) \\
& LAS Systems Tunnel (1) \\
10008 Upper Stage & MPCV Umbilical (1) \\
& Upper Stage Systems Tunnel (2) \\
& Cameras (2) \\
& GOX Vent Cover (1) \\
\hline \multirow{3}{*}{ Right SRB } & Systems Tunnel (1) \\
& Hold Down Posts (4) \\
& Aft BSM Nozzles (4) \\
& Fwd Attach Bracket Nub (1) \\
\hline \multirow{2}{*}{ Left SRB } & Systems Tunnel (1) \\
& Hold Down Posts (4) \\
& Aft BSM Nozzles (4) \\
& Fwd Attach Bracket Nub (1) \\
\hline
\end{tabular}

SLS 10008 SLS 28005 SLS 27005

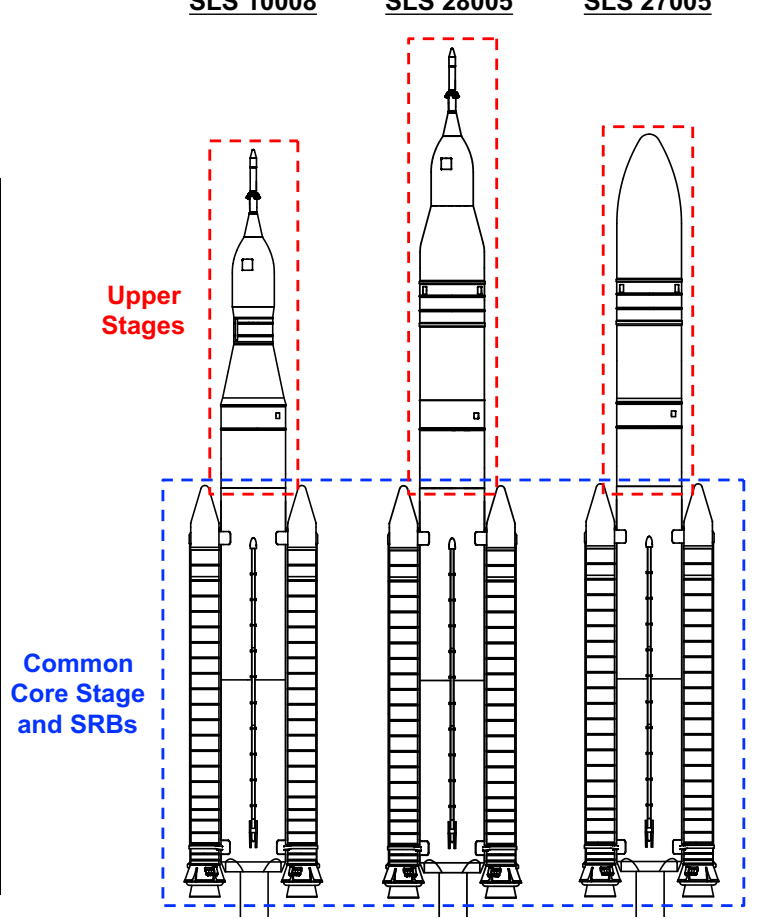

Figure 4. Details of the $1.75 \%$-scale SLS LOT models tested during Test 633 in the $14 \times 22$.

The model was also equipped with 158 surface pressure ports distributed around the circumference of the core and SRBs at six different axial cross sections of the vehicle, as shown in Figure 5. The lower part of the core stage contained 34 surface pressure ports, each SRB contained 48 surface pressure ports, and each different upper stage and nose contained 28 surface pressure ports, as summarized in Table 1.

Table 1. Surface pressure port summary for the SLS LOT model.

\begin{tabular}{|c|c|c|c|}
\hline Location & Number of Rings & Arrangement & Number of Ports \\
\hline Upper Stage / Nose & 2 & Every $30^{\circ}$ radially & 28 \\
\hline Lower Core Stage & 3 & Every $30^{\circ}$ radially & 34 \\
\hline Right SRB & 4 & Every $30^{\circ}$ radially & 48 \\
\hline Left SRB & 4 & Every $30^{\circ}$ radially & 48 \\
\hline Total & $\mathbf{1 3}$ & - & $\mathbf{1 5 8}$ \\
\hline
\end{tabular}




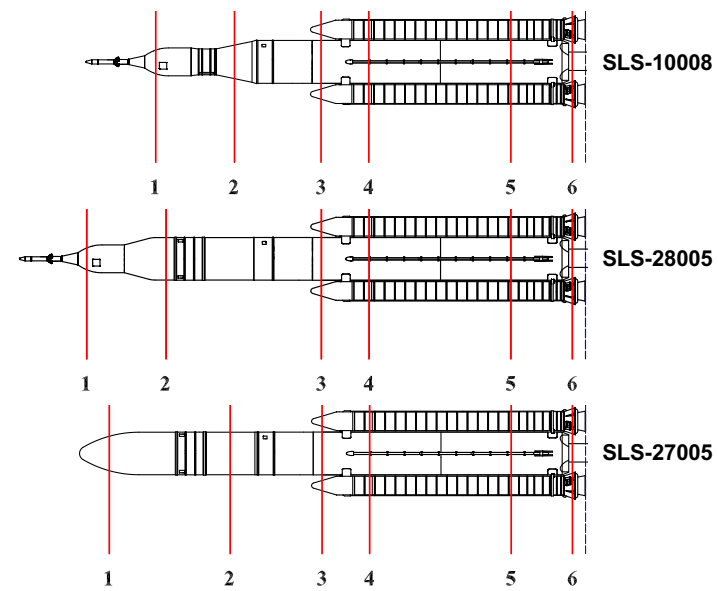

(a) Pressure row axial locations

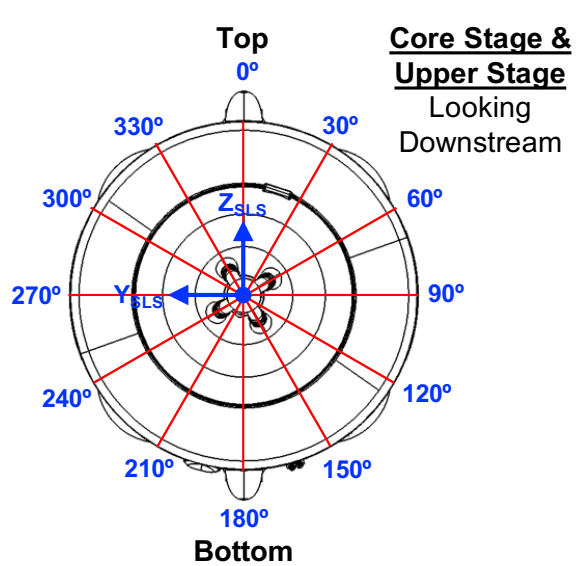

(b) Pressure row radial locations

Figure 5. Surface pressure port rows for the 1.75\%-scale SLS LOT models.

\section{B. Mobile Launch Platform and Launch Tower}

For the SLS Block 1 crew configuration, the same mobile launch platform and launch tower from the previous LOT test was used. However, for the SLS Block 1B crew and cargo configurations, an extension to the tower was needed because the launch tower was taller by essentially one tower level (Figure 6(a)). Furthermore, due to limitations in the vertical post motion range, data with the vehicle above the top of the launch tower were not able to be acquired in Test 609 . Therefore, for Test 633, an additional top-half tower was fabricated in order to acquire data with the vehicle above the top of the launch tower. Also, a one-level tower extension model part was fabricated that could be installed and removed to either the full tower or the half tower depending on the SLS vehicle configuration. The wind tunnel tower model parts used in Test 633 are shown in Figure 6(b).

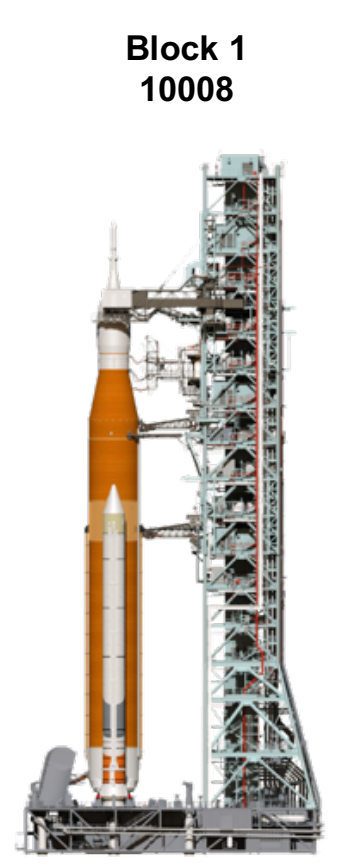

(a) Block 1 and Block 1B launch towers

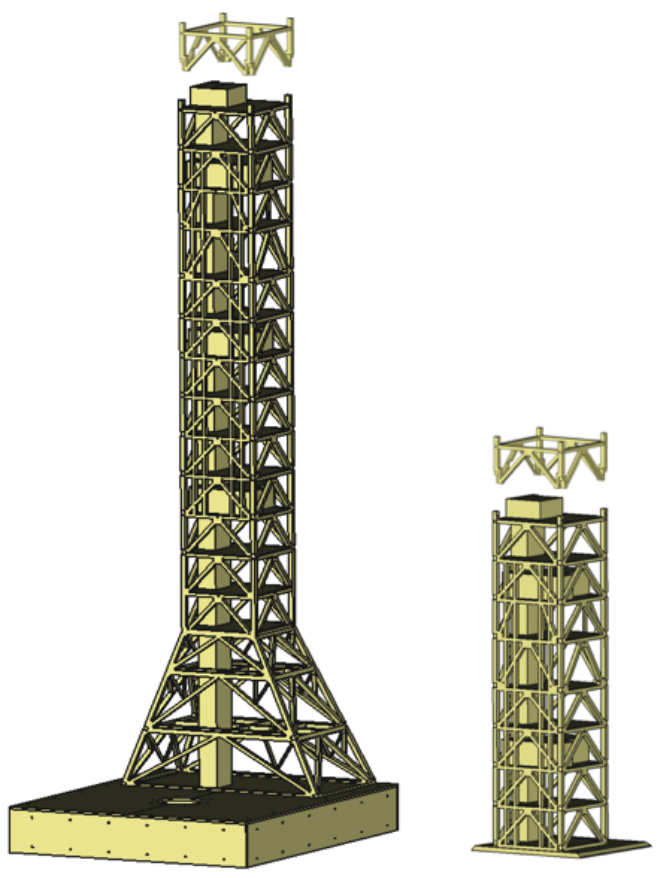

(b) WT full tower, half tower, and tower extensions

Figure 6. Details of the $1.75 \%$-scale MLP and Launch Tower tested during Test 633 in the $14 \times 22$. 


\section{Experiment Setup}

\section{A. Test Conditions}

The $14 \times 22$ is capable of running at dynamic pressures ranging from 10 to 120 psf, maximum air speeds of about $330 \mathrm{ft} / \mathrm{sec}$, and unit Reynolds numbers close to $2 \times 10^{6} / \mathrm{ft}$. Based on lessons learned from the previous SLS LOT test (Test 609), it was decided that the entire test matrix for Test 633 would be performed at a dynamic pressure of $50 \mathrm{psf}$ corresponding to a Mach number of about 0.18 . At the outset of the test, a series of dynamic pressure sweeps were performed (Table 2) to verify that similar results to Test 609 were obtained. The aerodynamic coefficients showed nonlinear trends below a dynamic pressure of 40 psf, but converge to nearly constant values above that. These were the same findings as Test 609, therefore, the test team was confident in the 50 psf choice.

Table 2. 14x22 freestream test conditions for the SLS LOT test.

\begin{tabular}{|c|c|c|c|}
\hline$q_{\infty}, \mathrm{psf}$ & $V_{\infty}, \mathrm{ft} / \mathrm{sec}$ & $M_{\infty}$ & $R e, 10^{6} / f t$ \\
\hline 10 & 92 & 0.082 & 0.566 \\
\hline 20 & 130 & 0.116 & 0.800 \\
\hline 30 & 160 & 0.142 & 0.980 \\
\hline 40 & 185 & 0.164 & 1.132 \\
\hline $\mathbf{5 0}$ & $\mathbf{2 0 7}$ & $\mathbf{0 . 1 8 3}$ & $\mathbf{1 . 2 6 7}$ \\
\hline 60 & 226 & 0.200 & 1.382 \\
\hline 70 & 244 & 0.216 & 1.492 \\
\hline 80 & 260 & 0.231 & 1.600 \\
\hline
\end{tabular}

\section{B. Boundary Layer Transition}

Because of the scale of the model, the Reynolds number of the flow on the vehicle is lower than in full scale flight. It is therefore important to devise a strategy to trip the boundary layer to make it turbulent, like on the flight vehicle. This is not easy for a slender launch vehicle that is pitched and rolled to large angles during testing. Capone et al. ${ }^{2}$ led a study of the most effective boundary layer tripping strategy for this type of vehicle and found that a uniform sprinkling of \#80-grit (approximately 0.008 inch size) on the entire body was the most consistent and predictable way to trip the boundary layer. The previous SLS LOT test used this strategy, therefore, the same strategy was used for Test 633. The photos in Figure 7 show close-ups of the model surface with \#80-grit uniformly sprinkled and secured to the model with all-purpose spray adhesive. When applying the adhesive and grit, the surface pressure ports on the model were protected, as can be noted in the photos. It is worth noting that a boundary layer tripping study was not performed during Test 633 , nor were there any methods employed to verify the state of the surface boundary layer during testing.
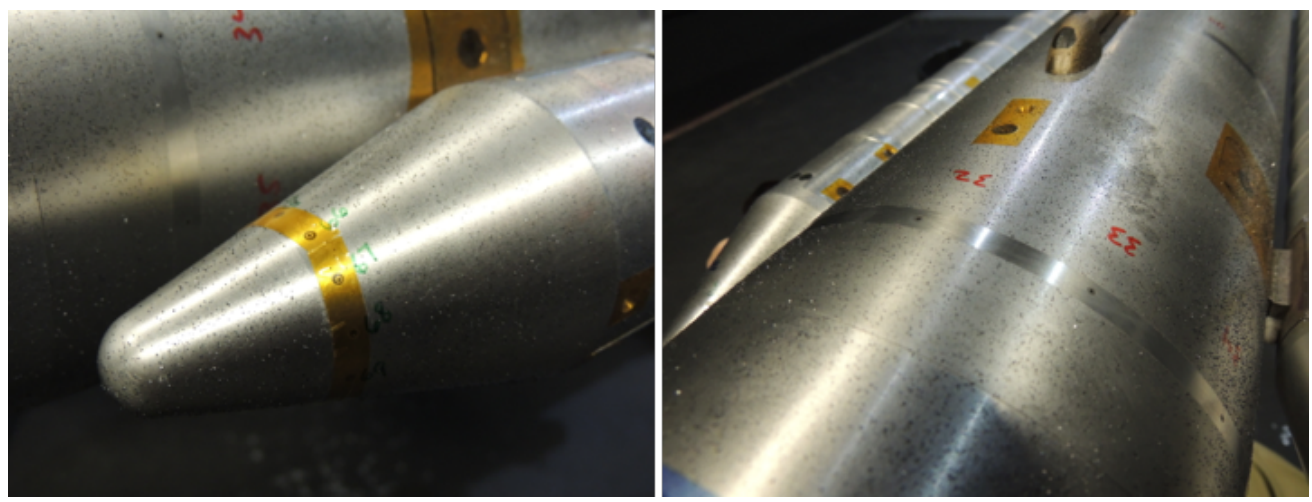

Figure 7. Uniformly sprinkled \#80-grit for boundary layer transition on the SLS LOT model. 


\section{Test Setup}

For the SLS LOT environment, the vehicle is subjected to ground wind loads from any direction before liftoff and also during liftoff and transition to forward flight. Before liftoff, for a given ground wind direction, the vehicle remains at a total angle of attack in the vicinity of $90^{\circ}$. As the vehicle lifts off the launch pad, it starts gaining longitudinal forward velocity, and the vehicle's total angle of attack decreases rapidly from $90^{\circ}$ to under $10^{\circ}$. As the vehicle transitions to forward flight, it is subjected to the freestream dynamic pressure in addition to the ground wind dynamic pressure. This is shown in Figure 8 as the $q_{\text {wind }}$ profile and the freestream $q_{\infty}$ impact the vehicle as it lifts off the launch pad.

In the wind tunnel, because it is a static simulation of the vehicle liftoff phase, it is not possible to simulate both the ground winds and freestream velocity simultaneously. Therefore, in order to capture the angle-of-attack effect for the vehicle while also measuring the tower interference aerodynamic effects, the wind tunnel test was designed in two parts. The first part of testing, or "transition aerodynamics" testing, of the vehicle in free-air was conducted to capture the angle-of-attack and angle-of-sideslip effects for the entire range from $-90^{\circ}$ to $90^{\circ}$. The second part of testing, or "tower interference" testing was conducted to measure the effects of the presence of the

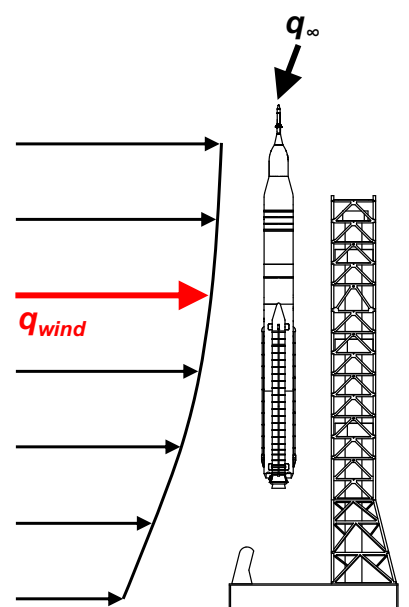

Figure 8. Diagram showing the SLS LOT environment. launch tower as a function of ground wind azimuth and launch height ratio. The vehicle stays at a total angle of attack of $90^{\circ}$ in this testing phase and so it is implicitly assumed that the tower effects do not depend on the vehicle angle of attack.

\section{Transition Aerodynamics Testing}

During this phase of testing, the SLS models were sting-mounted on the facility vertical support pitch strut. The pitch strut has a pitch angle range of $-10^{\circ}$ to $50^{\circ}$ (Figure $9(\mathrm{a})$ ). The sting is mounted to a motorized roll coupler that allows for continuous vehicle roll capability from $-180^{\circ}$ to $180^{\circ}$. The roll coupler is mounted to a custom pitch head offset adapter and could be set to two positions: $0^{\circ}$ and $45^{\circ}$, as shown in Figure 9 (b). The combination of the vertical post pitch strut and the pitch head offset adapter allowed for testing at a total angle of attack range from $-10^{\circ}$ to $50^{\circ}$ with the pitch head adapter in the $0^{\circ}$ offset position, and from $35^{\circ}$ to $95^{\circ}$ with the pitch head adapter in the $45^{\circ}$ position (Figure 10(a)). This allowed for an overlap of $15^{\circ}$ between the two pitch head offsets to ensure that there were no bias effects between the two setups. The vertical motion of the vertical post allowed for the model to remain, as much as feasible, in the center of the test section during testing for all angles of attack and angles of sideslip.
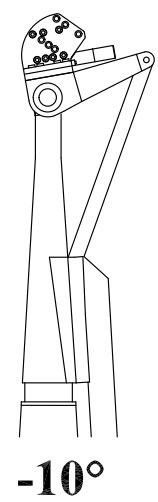

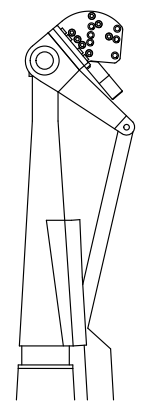

$\mathbf{5 0}$

(a) Pitch strut movement $-10^{\circ}$ to $50^{\circ}$
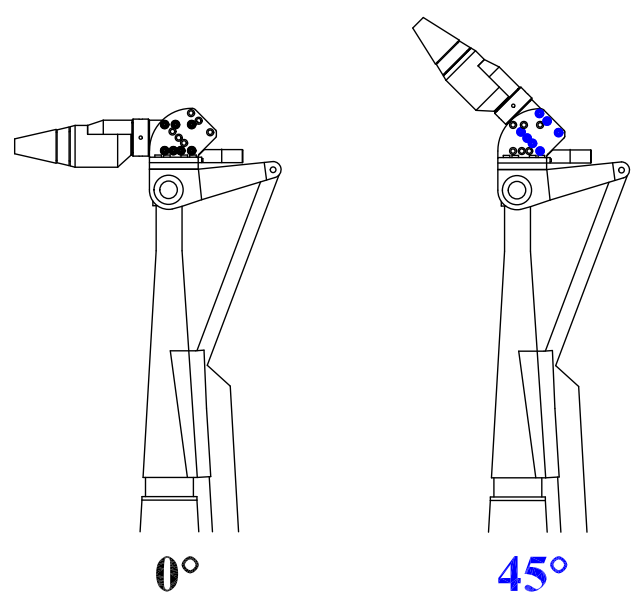

$45^{\circ}$

(b) Pitch head $0^{\circ}$ and $45^{\circ}$ offsets

Figure 9. Transition testing setup details of (a) pitch strut movement and (b) pitch head offsets. 


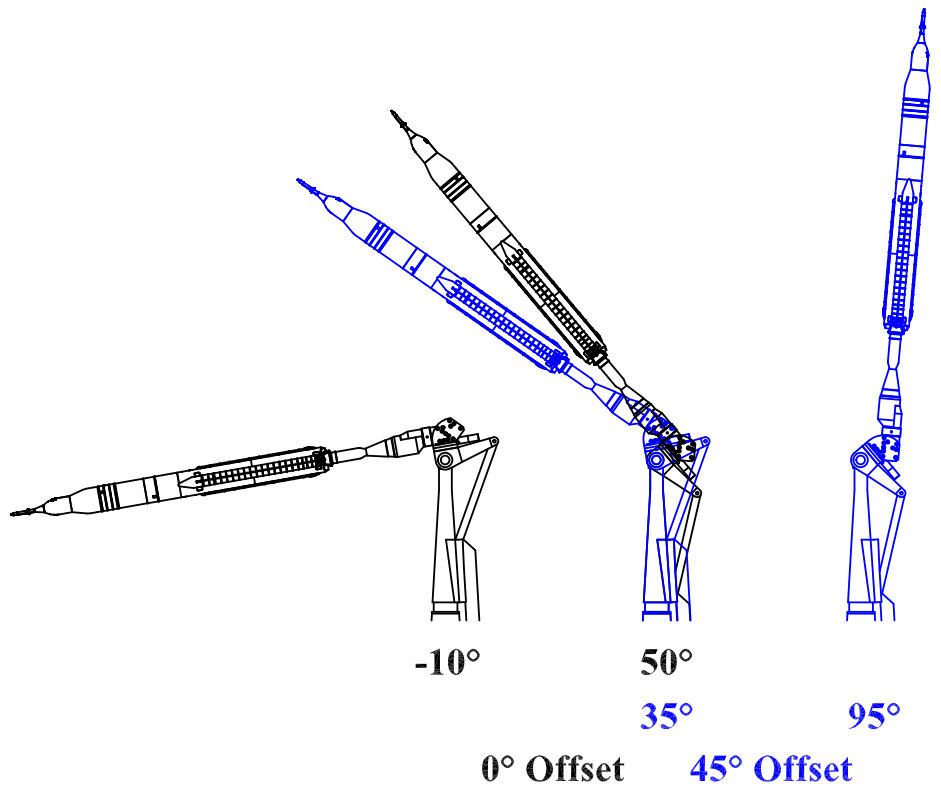

(a) Pitch angle coverage

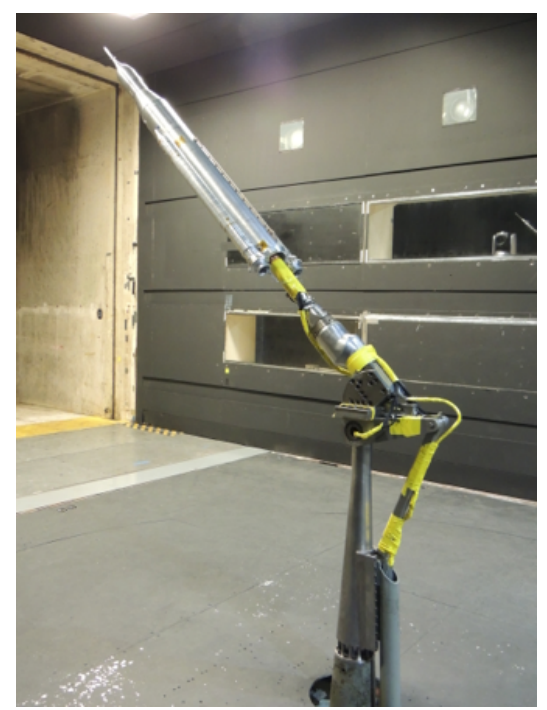

(b) Photo during transition testing

Figure 10. Transition testing pitch angle coverage with overlap range between $0^{\circ}$ and $45^{\circ}$ pitch head offsets.

For each SLS configuration tested in Test 633, free-air transition aerodynamic data were mostly acquired as pitch-angle sweeps at a constant roll angle. Using the two pitch head offsets, the pitch-angle sweep spanned the range from $-10^{\circ}$ to $90^{\circ}$ in $5^{\circ}$ increments and the sweeps were acquired at every $30^{\circ}$ in roll angle from $0^{\circ}$ to $330^{\circ}$. There were also a limited set of roll-angle sweeps from $0^{\circ}$ to $330^{\circ}$ in $15^{\circ}$ increments at constant pitch angles where the pitch angles were randomly chosen in order to fill in sparser areas of the test envelope. These data sets covered the total angle-of-attack range from $0^{\circ}$ to $90^{\circ}$ for all roll angles, which corresponds to an angle-of-attack and angle-of-sideslip range between $-90^{\circ}$ and $90^{\circ}$.

\section{Tower Interference Testing}

Tower interference effects are required for ground winds coming from all azimuthal directions and the launch vehicle is at various heights with respect to the tower, to simulate the vehicle lifting off until it clears the tower. The nondimensional launch height parameter, $h / L$, is used to describe the liftoff motion, where $h$ is the height of the vehicle above the launch pad and $L$ is the height of the launch tower. This is shown in Figure 11 where $h / L=0$ corresponds to the vehicle on the launch pad before liftoff and $h / L=1$ corresponds to the SRB nozzles clearing the top of the launch tower during liftoff.

During the tower interference testing phase, the vehicle is sting-mounted to the vertical post at a constant total angle of attack of $90^{\circ}$ (Figure 12). It can be tested without the launch tower, with the full launch tower, and with the top-half launch tower. When testing with the full launch tower, the vertical travel range of the facility vertical post allows the vehicle to be positioned at $h / L=0$ and almost as high as the top of the launch tower. For the Block 1B configurations, it could reach up to $h / L=0.91$ and for the Block 1 configuration, it could reach up to $h / L=0.95$. When testing with the top-half launch tower, the vertical post allowed testing from $h / L=0.60$ to $h / L=1.45$ for the Block $1 \mathrm{~B}$ configurations and from $h / L=0.62$ to $h / L=1.51$ for the Block 1 configuration. While the vehicle could be physically positioned lower, it was always tested at least 5 inches above the tunnel floor to avoid the tunnel floor boundary layer. Testing with both the full tower and the top-half tower allowed for an overlap range of around $0.3 h / L$ to ensure that there were no bias effects between the two setups.

Figure 13(a) shows a top view of the orientation of the vehicle with respect to the launch tower and MLP, and the definition of the wind azimuth angle, $\psi_{a z m}$. The launch tower is located to the north of the launch pad complex and therefore, a north wind $\left(\psi_{a z m}=0^{\circ}\right)$ blows through the tower and onto the bottom side of the vehicle imparting a positive normal force. A south wind $\left(\psi_{a z m}=180^{\circ}\right)$ blows onto the top side of the vehicle and then through the tower, imparting a negative normal force on the vehicle. West winds 


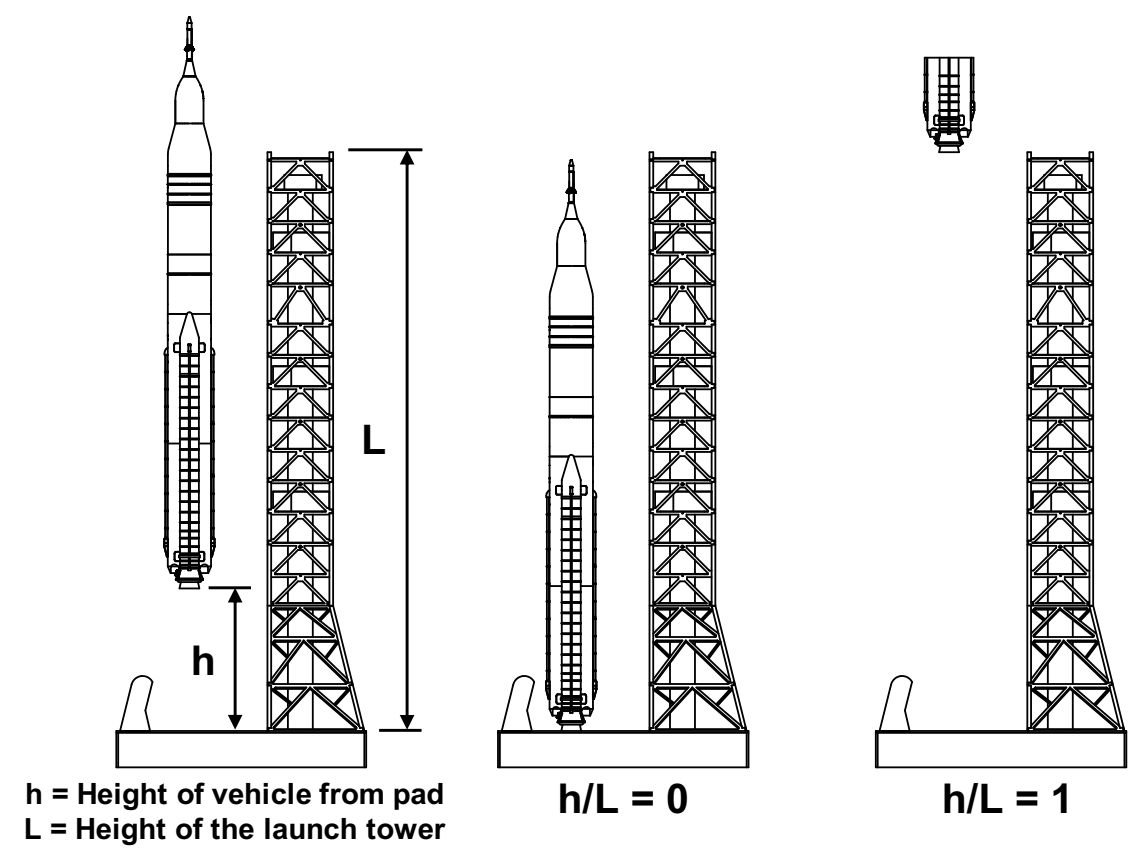

Figure 11. Definition of nondimensional launch height parameter, $h / L$.

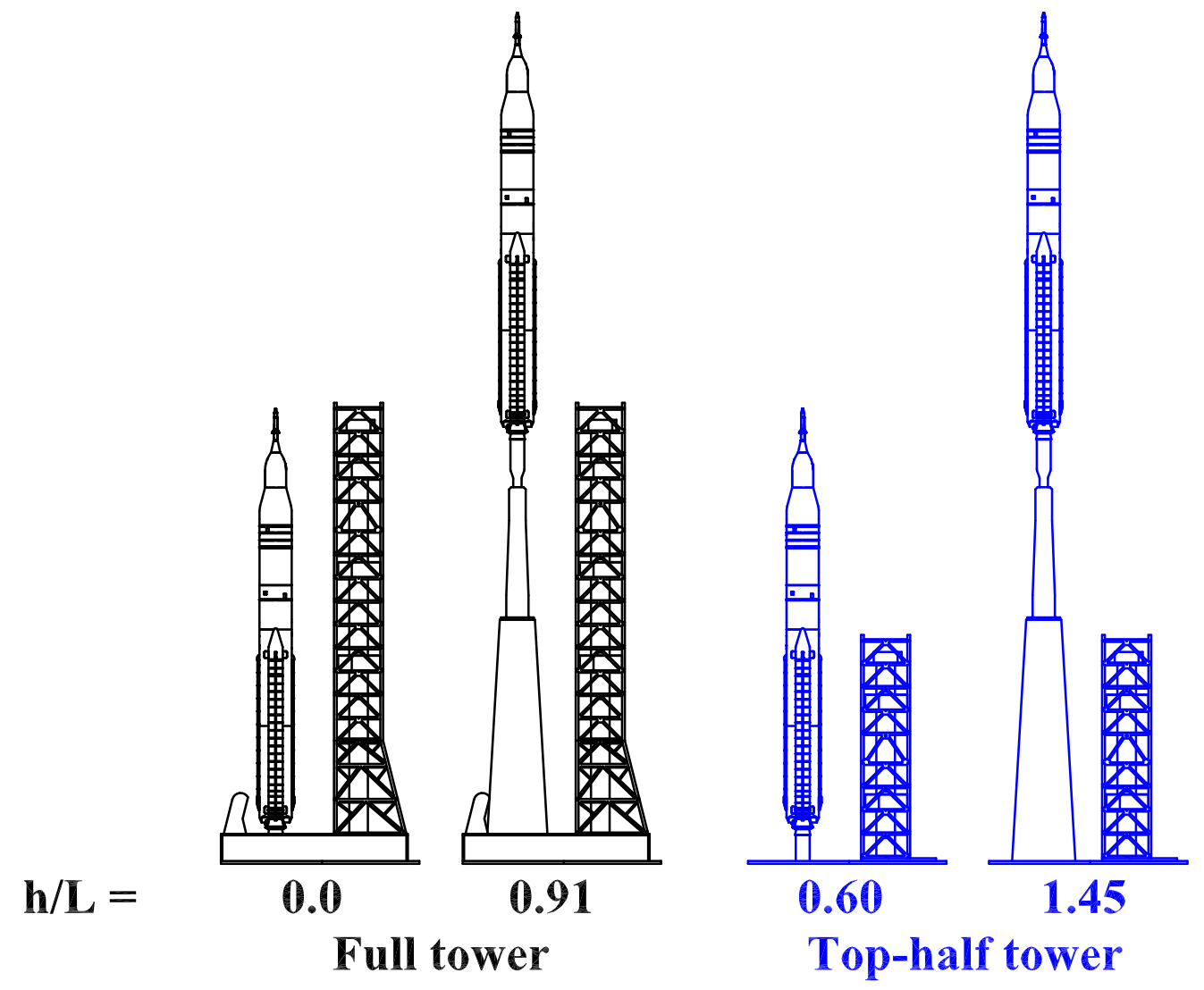

Figure 12. Tower interference testing setup with full tower \& top-half tower for the Block 1B crew configuration. 
$\left(\psi_{a z m}=270^{\circ}\right)$ would impart a positive side force, and east winds $\left(\psi_{a z m}=90^{\circ}\right)$ would impart a negative side force on the vehicle. In this setup, by definition, the vehicle roll angle with respect to the wind coincides with the wind azimuth angle.

During the tower interference testing phase, the vertical post support system was mounted on the facility cart turntable that has a rotating capability of $\pm 165^{\circ}$, for a total range of $330^{\circ}$, which results in a gap in wind azimuth angles that can be obtained in the tunnel. Because the model and tower are close to being symmetric about the $\mathrm{X}-\mathrm{Z}$ plane, the model was mounted such that the $30^{\circ}$ wind azimuth portion where data could not be acquired was placed for winds coming from the $\psi_{a z m}=75^{\circ}$ to $105^{\circ}$ azimuth angles, i.e., east winds (Figure 13(b)). To fill in the data gap, the westerly winds data acquired for the $255^{\circ}$ to $285^{\circ}$ wind azimuth angles were mirrored to the easterly winds using symmetry assumptions. This ensured that the most important orientations, north and south winds, were able to be acquired.

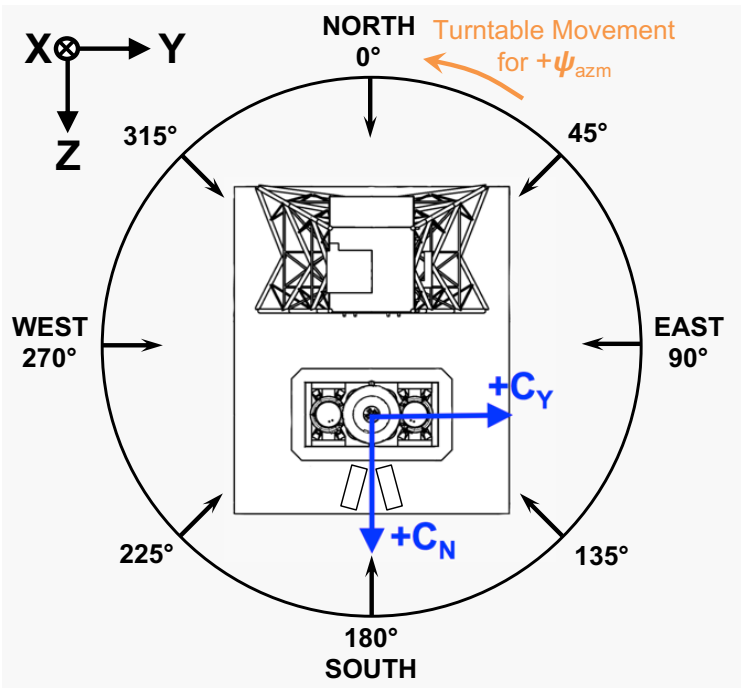

(a) Wind azimuth angle definition, $\psi_{a z m}$

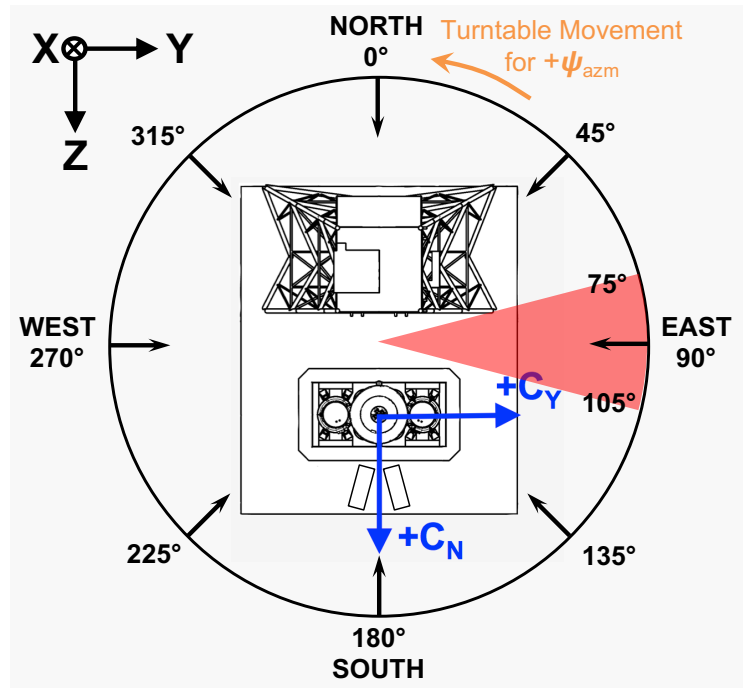

(b) Deadband due to turntable limitation

Figure 13. Top view of the SLS vehicle on the MLP showing the (a) wind azimuth angle definition and (b) deadband due to turntable limitation.

For each SLS configuration tested in Test 633, aerodynamic data in this testing phase were mostly acquired as wind azimuth sweeps at a constant $h / L$, where data were acquired in both positive and negative azimuthal directions in increments of $10^{\circ}$. Positive wind azimuth sweeps therefore started at $\psi_{a z m}=110^{\circ}$, increasing to $\psi_{a z m}=360^{\circ}$ and then following through to $\psi_{a z m}=70^{\circ}$; and a negative azimuth sweep started at $\psi_{a z m}=70^{\circ}$, decreasing to $\psi_{a z m}=0^{\circ}$ and following through all the way down to $\psi_{a z m}=110^{\circ}$. There were also a limited set of $h / L$ sweeps at a constant wind azimuth angle, where the height of the vehicle was swept from the minimum $h / L$ achievable in the test setup (full tower or top-half tower) up to the maximum $h / L$ achievable in increments of $0.1 h / L$, or swept in the opposite direction from maximum down to the minimum. These data sets covered all wind azimuth angles, except for the few easterly azimuth angles that could not be acquired due to turntable limitations, and $h / L$ from 0 to about 1.5 depending on the vehicle and tower configuration.

To compute tower interference effects in an incremental fashion, data needed to be acquired without the launch tower, and then with the launch tower (full tower and top-half tower). The difference between the no-tower and tower data sets constitutes the tower effect increment. Therefore, the same wind azimuth sweeps and $h / L$ sweeps described earlier were acquired in the test setups without the tower (vehicle alone), and with the tower (full tower or top-half tower). This made it easier when computing the tower effects increments and also removed any possible bias effects due to the position of the model in the test section.

Figure 14 shows photos of each of the SLS configurations in each of the testing phases. The testing order consisted of transition aerodynamics testing on the pitch strut mechanism, followed by vehicle-alone (no tower) liftoff testing, then tower interference testing with the full tower, and finally tower interference testing with the top-half tower. The three SLS configurations were tested in each setup first before moving onto the next test setup. 

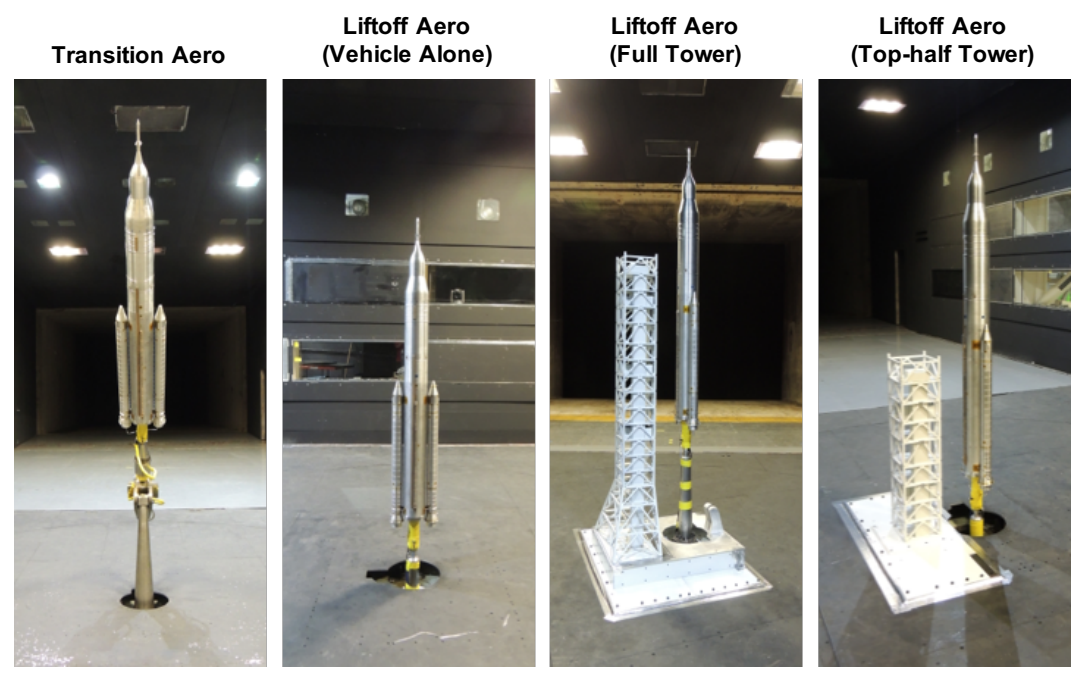

(a) Block 1B crew configuration
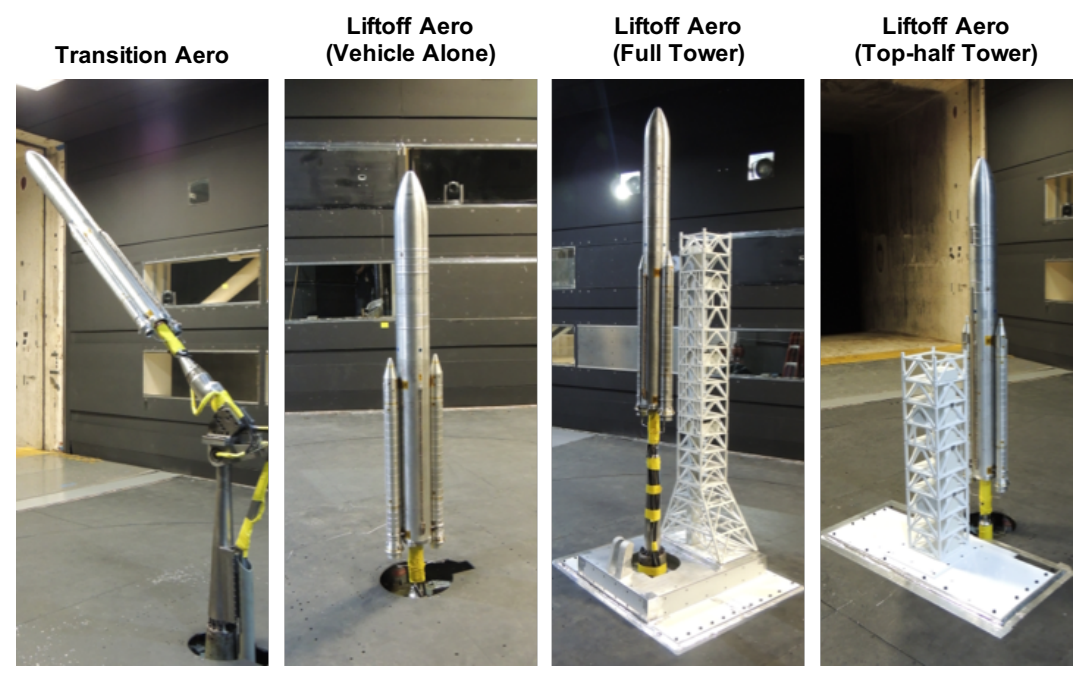

(b) Block 1B cargo configuration
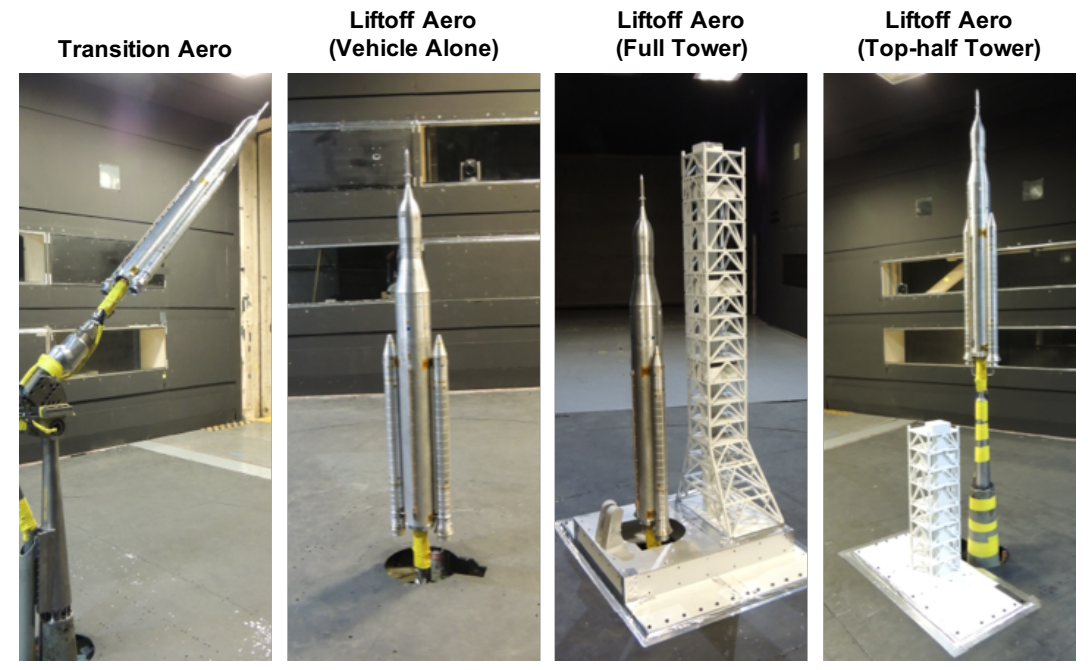

(c) Block 1 crew configuration

Figure 14. Sample test photos of each SLS configuration in the various testing phases. 


\section{Improved Testing Methods}

This section will describe some of the improved testing methods that were implemented in Test 633 based on lessons learned from Test 609. Furthermore, new SLS program requirements necessitated the implementation of a novel and unique force measurement system for the SRBs.

\section{A. Stiffer Sting and Balance Combination}

In the previous SLS LOT test, severe sting-balance-model dynamics were encountered due to a force measurement and model support system that were too flexible. This prevented data acquisition during the transition testing phase in the total angle-of-attack region between $30^{\circ}$ and $60^{\circ}$, and also prevented data acquisition during the tower interference testing phase at wind azimuths around $30^{\circ}$ and $330^{\circ}$. Video footage of the dynamic events were captured throughout the testing and played a critical role in identifying the cause of the undesirable dynamics. The video footage showed low frequency oscillations at less than $10 \mathrm{~Hz}$, which have historically been attributed to stings and balances with too much flexibility. ${ }^{5}$ The location of the model center of gravity (CG) was likely another contributing factor.

To test these hypotheses, a first-order finite-element modal analysis was performed, where the location of the model CG in relation to the balance moment center (BMC) representative of a given model configuration, the sting diameter, and the balance diameter were varied. The sting-balance-model system was simplified in the 3-D modeling software for convenience without adversely impacting the interpretation of the results. The design and results from the study are shown in Table 3. The combination of a 1.5-inch sting diameter and a 1.5-inch balance diameter (Balance 756) are representative of the sting-balance configuration used during Test 609. From the study, it was noted that the first frequency mode was affected by sting diameter and model CG location, and the second frequency mode was affected by sting diameter and balance. Therefore, a larger diameter sting $(2.5 \mathrm{inch})$ and larger diameter balance $(2.0 \mathrm{inch})$ combination were selected as an appropriate solution to the dynamics problem and would more than double the 1st mode natural frequency of the original sting-balance system. Relocation of the model CG was not considered during this process since many of the same model parts were being reused for Test 633 .

Table 3. Results of Sting-Balance-Model Finite Element Modal Analysis.

\begin{tabular}{|c|c|c|c|c|}
\hline $\begin{array}{c}\text { Model CG Location } \\
\text { (core diameters } \\
\text { behind BMC) }\end{array}$ & $\begin{array}{c}\text { Sting } \\
\text { Diameter } \\
\text { (inches) }\end{array}$ & $\begin{array}{c}\text { Balance } \\
\text { Diameter } \\
\text { (inches) }\end{array}$ & $\begin{array}{c}\text { 1st Mode } \\
\text { Frequency } \\
(\mathrm{Hz})\end{array}$ & $\begin{array}{c}\text { 2nd Mode } \\
\text { Frequency } \\
(\mathrm{Hz})\end{array}$ \\
\hline \multirow{3}{*}{$\mathbf{0 . 2 2}$} & $\mathbf{1 . 5}$ & $\mathbf{1 . 5}$ & $\mathbf{5 . 3 9}$ & $\mathbf{2 0 . 2 1}$ \\
\cline { 2 - 5 } & 2.0 & 1.5 & 8.89 & 24.52 \\
\cline { 2 - 5 } & \multirow{2}{*}{$\mathbf{2 . 5}$} & 2.0 & 9.39 & 30.95 \\
\cline { 3 - 5 } & 1.5 & $\mathbf{2 . 0}$ & 11.95 & 29.09 \\
\hline \multirow{4}{*}{0.65} & 2.0 & 1.5 & 7.11 & $\mathbf{3 5 . 5 3}$ \\
\cline { 2 - 5 } & \multirow{2}{*}{2.5} & 2.0 & 12.11 & 24.59 \\
\cline { 2 - 5 } & & 2.0 & 12.47 & 32.27 \\
\cline { 3 - 5 } & & & 16.83 & 28.27 \\
\hline
\end{tabular}

A new sting was designed and fabricated to have a 2.5-inch diameter, but the aft end needed to taper back down to a 1.5-inch diameter to interface with the tunnel roll coupler hardware and vertical post adapter. The front end of the sting was made to accept the taper of a 2.0-inch diameter balance. Additionally, a new balance drawnut and new model balance block were designed and fabricated to interface with the new sting-balance system. The selection of a 2.0-inch diameter balance that would best suit the objectives and requirements of the test was done in collaboration with force measurement engineers at Langley. The force measurement engineers were able to assess a large pool of balance candidates and were able to narrow down the number of potential candidates based on the expected test loads. Two main criteria, measurement 
resolution for the expected test loads and global stiffness of the balance, were used to make the final selection of the VST-15 balance for use in Test 633 . Because strain-gauge balances rely on deflections to increase output of a strain-gauge bridge, stiffness is usually inversely proportional to measurement resolution. However, the VST-15 provided more global stiffness and comparable or better measurement resolution at the expected test loads than the 756 balance as shown in Table 4. This was a nonintuitive and unexpected positive benefit to the test team.

Table 4. Balance resolution and stiffness comparison at SLS test loads between 756 and VST-15 balances.

\begin{tabular}{|c|c|c|c|c|}
\hline \multirow{2}{*}{} & \multicolumn{2}{|c|}{$\begin{array}{c}\text { Output at Test } \\
\text { Loads, } m V / V\end{array}$} & \multicolumn{2}{c|}{$\begin{array}{c}\text { Deflection at } \\
\text { Test Loads, } d e g\end{array}$} \\
\cline { 2 - 5 } & 756 & VST-15 & 756 & VST-15 \\
\hline Axial Force & 0.523 & $\mathbf{0 . 5 6 6}$ & 0.0 & $\mathbf{0 . 0}$ \\
\hline Side Force & 0.411 & $\mathbf{0 . 7 7 8}$ & 0.028 & $\mathbf{0 . 0}$ \\
\hline Normal Force & 0.391 & $\mathbf{0 . 5 9 2}$ & 0.055 & $\mathbf{0 . 0 1 7}$ \\
\hline Rolling Moment & 0.294 & $\mathbf{0 . 3 2 1}$ & 0.252 & $\mathbf{0 . 1 2 5}$ \\
\hline Pitching Moment & $\mathbf{0 . 9 3 4}$ & 0.523 & 0.532 & $\mathbf{0 . 2 6 8}$ \\
\hline Yawing Moment & $\mathbf{1 . 8 2 7}$ & 1.267 & 0.572 & $\mathbf{0 . 2 9 7}$ \\
\hline
\end{tabular}

Finally, a comparison between the original and new sting-balance system is shown in Figure 15. During installation of the sting-balance-model system in the $14 \times 22$, a static modal response test was performed by impacting the system at various locations in order to measure the natural frequencies of the system, and in particular, to verify the sting and balance natural frequencies as predicted in the modal analysis described earlier. Figure 16 shows the spectral density data of the VST-15 normal and side force gauges when the model was hit at the BMC, at the roll coupler, and at the pitch mechanism. The measured 1st mode balance frequency is around $6-7 \mathrm{~Hz}$ and the measured 1st mode sting frequency is around $13-15 \mathrm{~Hz}$. The

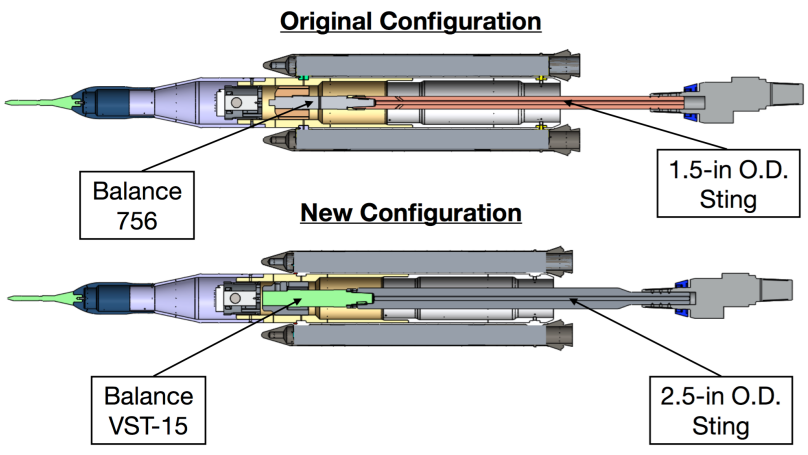

Figure 15. Comparison between original (Test 609) and new (Test 633) sting-balance system.

measured sting 1st mode frequency compares very well to the pretest prediction shown in Table 3, thus verifying the pretest modal analysis as a viable tool for predicting the natural response of a sting-balance-model system.

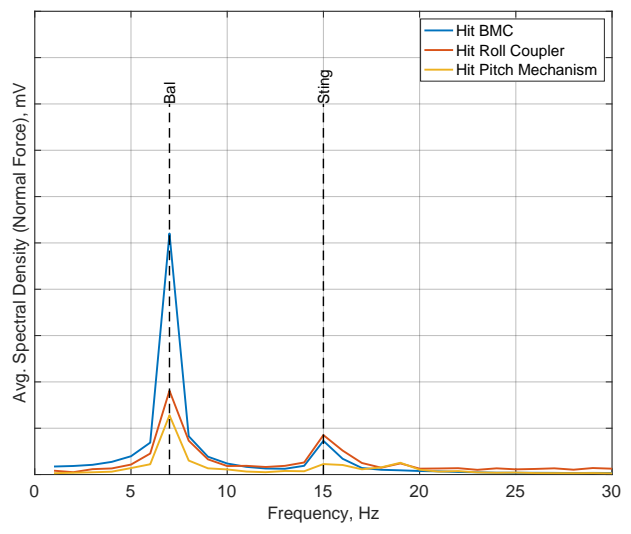

(a) Balance Normal Force

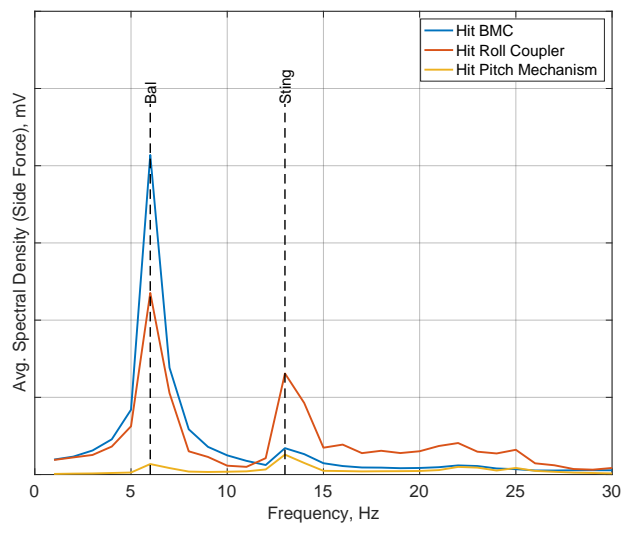

(b) Balance Side Force

Figure 16. Static modal response test of sting-balance-model system used in Test 633 . 


\section{B. Separate SRB Force \& Moment Measurements}

In addition to solving the dynamics problem encountered during the previous SLS LOT test, the SLS program also desired the ability to measure forces and moments on each SRB separately from the main balance that measured forces and moments on the whole vehicle. This was challenging because of the relatively small size of the SRBs and its proximity to the core stage of the model. One option considered during the development of the SRB force measurement system was the fabrication of custom SRB attach brackets with instrumented flexures. While this option could have been tailored to the expected loads, lead time and budgetary concerns made the option prohibitive. Therefore, commercially-available options for measuring multiaxis loads were considered and ultimately selected.

A novel and unique force measurement system (FMS) was devised where two miniature pancake-style six-component load cells were installed in each SRB. These load cells were attractive for various reasons: the compact design allowed it to fit inside the SRBs, the load cells could measure all six F\&M components, and factory calibrations for each load cell were provided upon delivery. The combination of the two load cells in each SRB essentially acted as a separate six-component balance with the moment center at the midpoint between the two load cells. An in situ system calibration could be performed after model assembly to remove any misalignment effects encountered during assembly. More details about the load cells and the system calibration are provided in subsequent sections.

While this load cell concept seemed viable, the challenge was how to integrate them into an existing model with minimal modifications. After careful collaboration between the model designers and the force measurement engineers, a design concept was devised where each load cell would be sandwiched between an SRB attach bracket and a mount pad in the SRB as shown in Figure 17 using the right SRB aft attach bracket as an example. Provisions were made to allow the routing of the load cell cable underneath the mount pad and a small gap between the SRB and the attach bracket was included to preserve the load cell measurement metric break. Due to space constraints inside the SRBs, the load cells were mounted in such a way that the SRB attach bracket (and the core stage) were on the metric side of the transducer, while the SRB itself was on the nonmetric side of the transducer. While this is reverse from conventional practice, the measurements were corrected in the data reduction software.

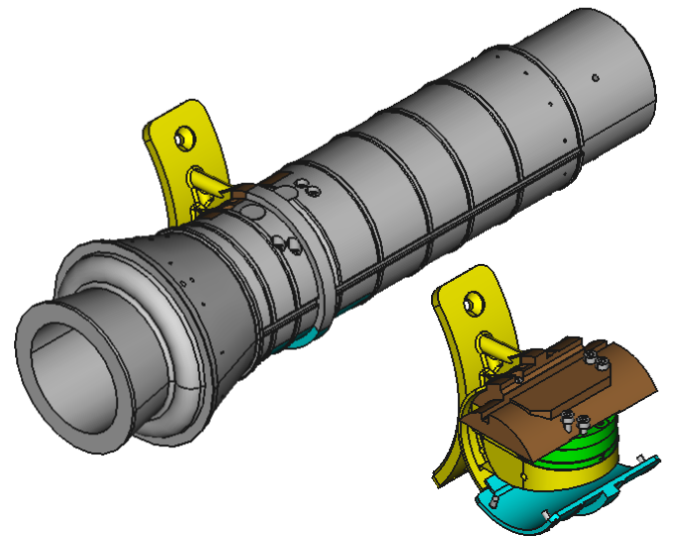

(a) RSRB aft attach bracket

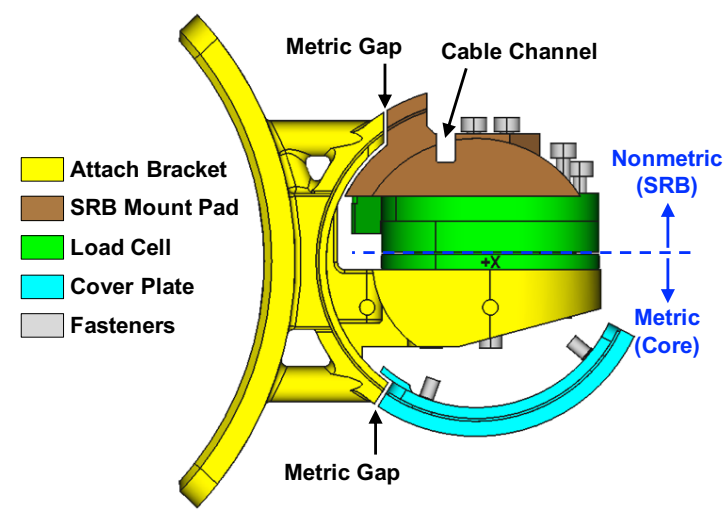

(b) Load cell integration

Figure 17. Integration of subminiature 6-component load cells into SRB attach brackets.

The load cell mounting brackets were fabricated out of Inconel 625 using in-house additive manufacturing capabilities and were designed such that minimal modifications were required to the existing LOT model and in particular the SRB bodies. The brackets were 3D-printed because of their unique shape and because the test team wanted to preserve certain geometric features on the bracket between the SRBs and the core. The mounting surface to the load cell was machined after printing to allow for a stable and repeatable installation. Figure 18 shows a photo of the SRB attach brackets and also shows that the metric gap was preserved during model assembly, even with slight misalignments in the installation of the load cells due to fabrication tolerances. 


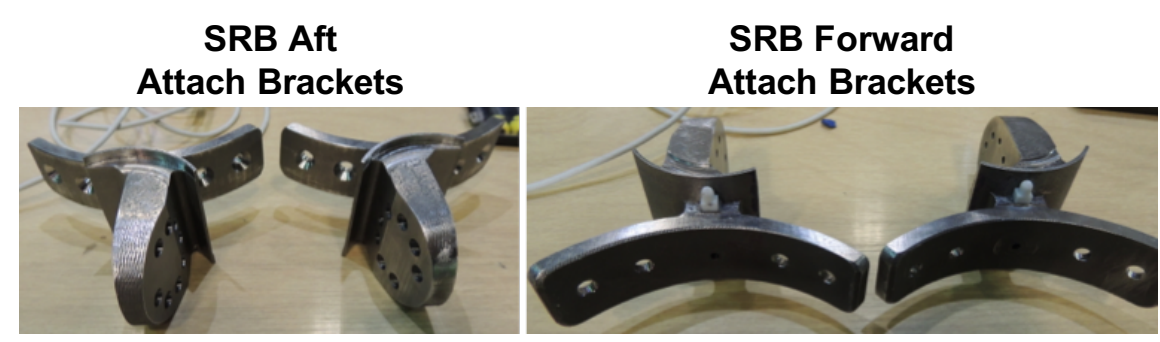

(a) SRB attach bracket hardware

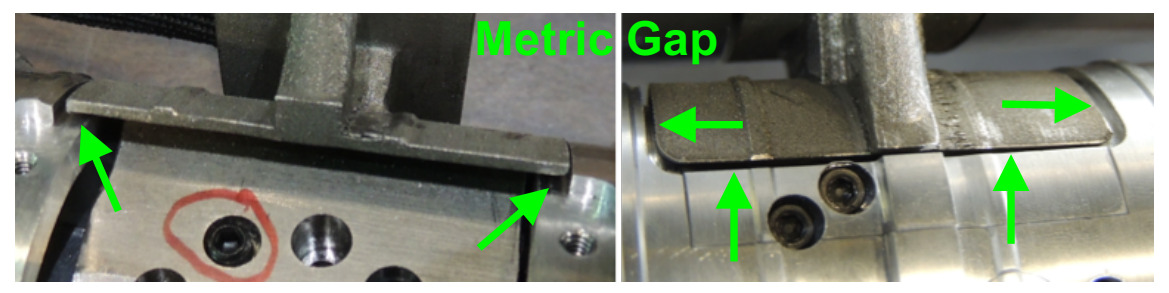

(b) Metric gap around SRB attach bracket

Figure 18. SRB attach brackets 3D-printed out of Inconel 625 and preservation of metric gap around brackets.

\section{Smoke Flow Visualization Upgrade}

While smoke flow visualization was performed in the previous SLS LOT test, the smoke wand and associated hardware were not the best quality and the smoke stream was not strong enough to persist downstream past the model. Therefore, a commercial smoke flow generator system was purchased and used for Test 633 . This system used heated propylene glycol for the smoke stream and compressed air to shoot the stream out of the front fitting on the long smoke wand (Figure 19). This system was a major improvement as the smoke stream was very tight and clear and persisted well downstream past the model and turntable.

Furthermore, the bare metal of the MLP and the facility turntable made it difficult to acquire photographs with enough contrast to see the smoke stream clearly. Therefore, the MLP and entire turntable were painted a flat black for the smoke flow visualization sessions. While it would have been desirable to also paint the launch tower black, the white powder coat on the tower would have made it difficult for new paint to adhere to it and the complex truss structure would have also made it difficult to paint. The vehicle model was also not painted because aerodynamic F\&M data were still acquired after the smoke flow sessions and the model surface needed to stay consistent throughout the test.

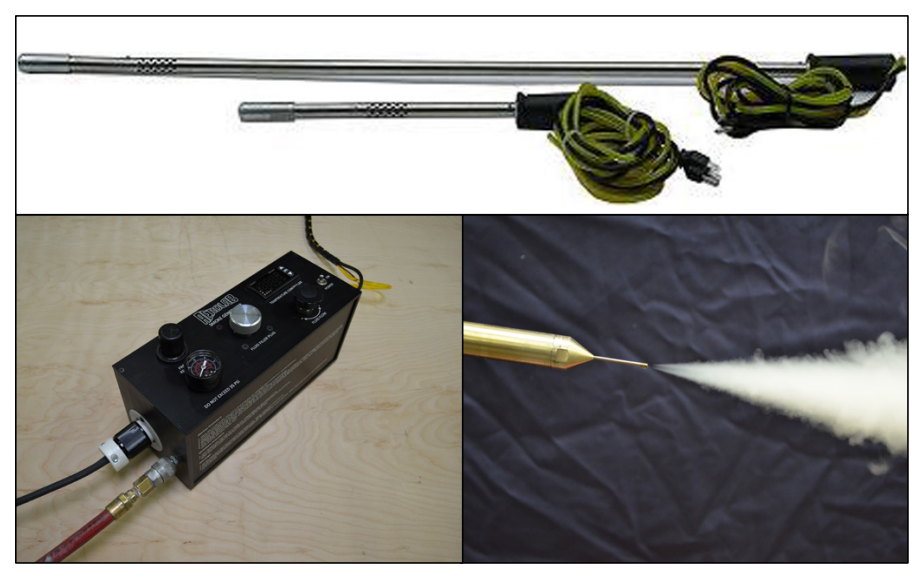

(a) Smoke generator system

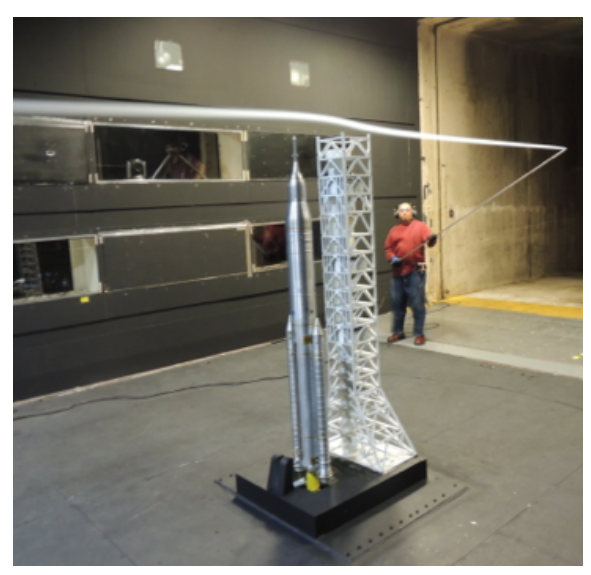

(b) Smoke stream, painted floor and MLP

Figure 19. Improvements to smoke flow visualization in the $14 \times 22$ for the SLS LOT test. 


\section{Measurements and Instrumentation}

\section{A. Full Vehicle and SRB Forces and Moments}

In order to measure the forces and moments on the full vehicle and each SRB separately, a total of five 6-component force transducers were used simultaneously during the SLS LOT Test 633 in the $14 \times 22$. The total FMS suite shown in Figure 20 consisted of the main strain-gauge balance (VST-15), which measured the full vehicle F\&M and the four load cells, which measured the SRB F\&M. The combination of the two load cells in each SRB essentially acted as a separate six-component balance. Note that the model in Figure 20 is shown from the bottom view. Details of these force transducers are provided in the following sections.

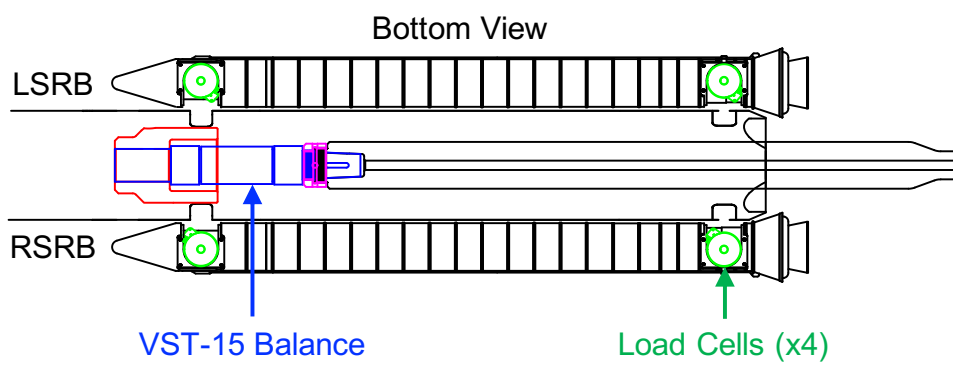

Figure 20. Total force measurement system suite for the SLS LOT model used in Test 633 at the $14 \times 22$.

\section{VST-15 Balance}

The VST-15 balance is a six-component strain-gauge balance made out of Vascomax C300 that measures 2.06 inches in diameter and 13.15 inches in length. Figure 21 shows an outline drawing and photo of the VST-15 balance. The balance is tapered on the nonmetric end to install to the 2.5 -inch diameter sting and secured with a drawnut, while the metric end installs to the SLS LOT model via a 0.625 -inch diameter dowel pin. The sting-to-balance fit was inspected prior to the test with $100 \%$ surface contact between the balance taper and the front end of the sting. A custom balance key was fabricated to ensure a perfect fit between the balance and the sting, ensuring that no play would exist in the support hardware. Similarly, a custom dowel pin was fabricated to ensure a tight fit between the balance and the SLS LOT model. The balance was calibrated before the test in December of 2016 and the results of the calibration are shown in Table 5 .

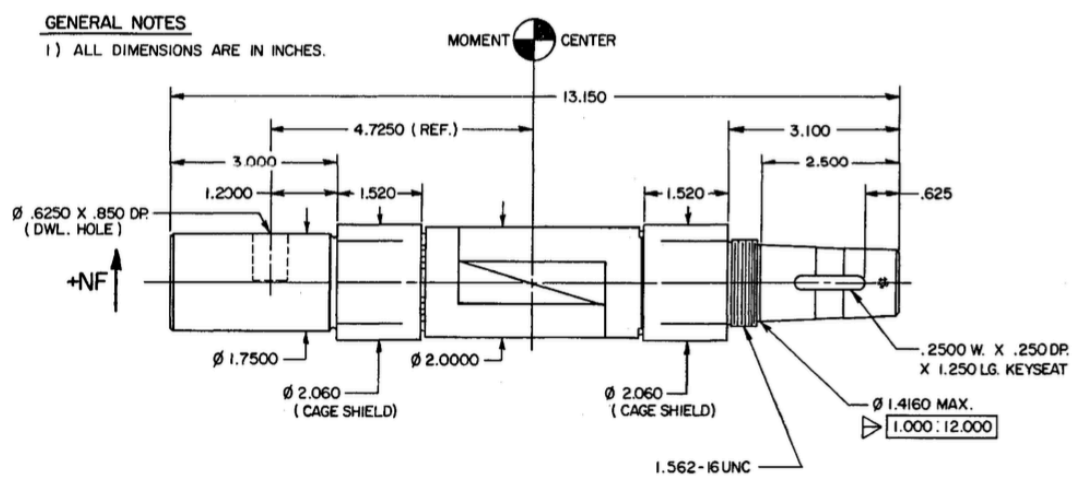

(a) Outline drawing

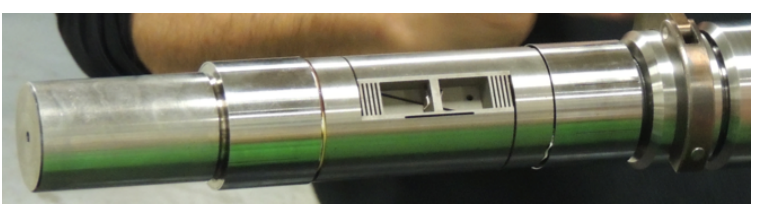

(b) Photo

Figure 21. VST-15 balance used in SLS LOT Test 633 in the $14 \times 22$. 
Table 5. VST-15 balance calibration summary.

\begin{tabular}{|c|c|c|c|}
\hline Balance & Calibration & \multicolumn{2}{|c|}{$2 \sigma$ Calibration Error } \\
\cline { 3 - 4 } Component & Load Range & \%-Full Scale & Engineering Units \\
\hline Axial Force & $\pm 150 \mathrm{lbf}$ & 0.15 & $\pm 0.23 \mathrm{lbf}$ \\
\hline Side Force & $\pm 150 \mathrm{lbf}$ & 0.25 & $\pm 0.38 \mathrm{lbf}$ \\
\hline Normal Force & $\pm 500 \mathrm{lbf}$ & 0.13 & $\pm 0.65 \mathrm{lbf}$ \\
\hline Rolling Moment & $\pm 2,000 \mathrm{in}-\mathrm{lbf}$ & 0.17 & $\pm 3.4 \mathrm{in}-\mathrm{lbf}$ \\
\hline Pitching Moment & $\pm 6,000 \mathrm{in}-\mathrm{lbf}$ & 0.06 & $\pm 3.6 \mathrm{in}-\mathrm{lbf}$ \\
\hline Yawing Moment & $\pm 2,000 \mathrm{in}-\mathrm{lbf}$ & 0.13 & $\pm 2.6 \mathrm{in}-\mathrm{lbf}$ \\
\hline
\end{tabular}

\section{ATI-IA Mini45-ERA Load Cells}

The Mini45-ERA Force/Torque transducer (Figure 22) from ATI Industrial Automation (ATI-IA) is a subminiature, pancake-style, 6-component F\&M load cell typically used in industrial robotics applications. It has a compact, low-profile design with high load capacity and is made from high yield-strength stainless steel with maximum allowable overload values that are nearly 20 times the rated capacities. The load cell is about 1.77 inches in diameter with about a 0.62 inch thickness. The silicon strain gauges provide a high signal-to-noise ratio and high measurement resolution. In short, the load cells are very stiff, but still very sensitive to strain. The load cells were also ordered with the -ERA option meaning that the load cell cable was strain relieved and exited the transducer at a right angle. This helped the integration of the load cells into the SRBs. The load cells were oriented in the SRBs such that the load cell X, Y, and Z axes were coincident with the SRB X, Y, and Z axes. A 6-component factory calibration was also provided from the manufacturer for each load cell and the quoted calibration accuracv is shown in Table 6.

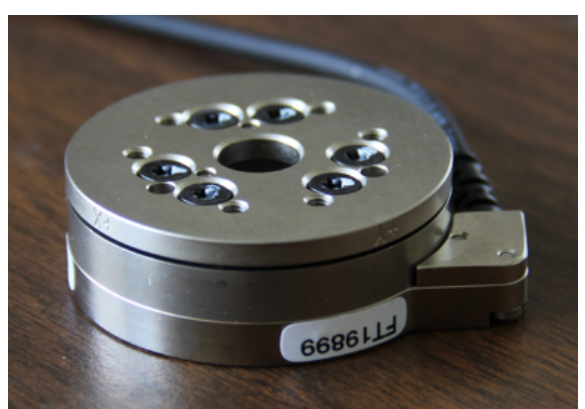

(a) Photo

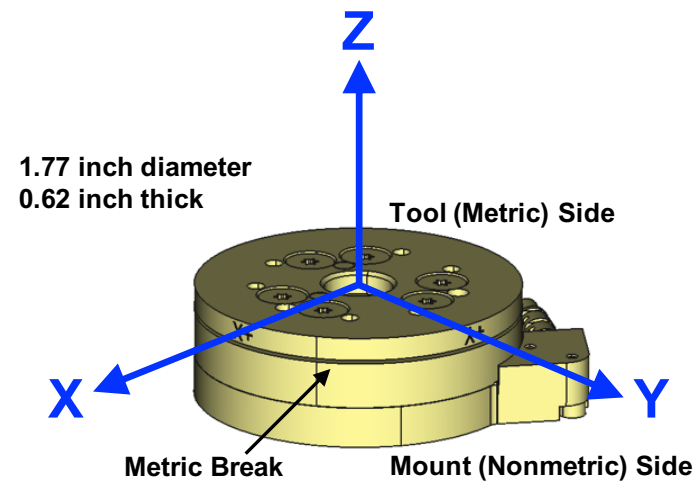

(b) Axes definition

Figure 22. ATI Industrial Automation Mini45-ERA 6-component F\&M load cell.

The load cells were also ordered without manufacturer-supplied signal amplification and conditioning electronics due to space constraints inside the SRBs. This meant that the load cells were comprised of halfbridge circuits, which could not be directly connected to nanovolt range meters. Therefore, special cabling (Figure 23(a)) were produced to arrange bridge completion circuits near the load cell connector inside the SRBs. Having the completion circuits close to the load cells inside the SRBs preserved the high signalto-noise ratio of the load cells. Precision resistors were used to provide bridge completion with minimum temperature effects and six resistor pairs, one for each component channel, were packaged inside the cable.

There were a total of four cables, one for each load cell. One end of the cable had a connector that would mate with the supplied load cell cable connector, while the other end of the cable had a small Nanonics connector that made it easier for the cable to exit the SRB and mate with the wind tunnel electronics. There was also a small concern of air flow inside the SRBs and whether that would affect the load cell performance. Therefore, loose fitting Kapton rings (Figure 23(b)) were placed over each load cell to mitigate this concern. 
Table 6. ATI-IA Mini45-ERA load cell calibration summary.

\begin{tabular}{|c|c|c|c|c|}
\hline Load Cell & Calibration & Single Axis & \multicolumn{2}{|c|}{$2 \sigma$ Measurement Uncertainty } \\
\cline { 4 - 5 } Component & Load Range & Overload & \%-Full Scale & Engineering Units \\
\hline Force in X-direction & $\pm 60 \mathrm{lbf}$ & $\pm 1,100 \mathrm{lbf}$ & 1.25 & $\pm 0.75 \mathrm{lbf}$ \\
\hline Force in Y-direction & $\pm 60 \mathrm{lbf}$ & $\pm 1,100 \mathrm{lbf}$ & 1.00 & $\pm 0.60 \mathrm{lbf}$ \\
\hline Force in Z-direction & $\pm 120 \mathrm{lbf}$ & $\pm 2,300 \mathrm{lbf}$ & 0.75 & $\pm 0.90 \mathrm{lbf}$ \\
\hline Moment about X-axis & $\pm 80 \mathrm{in}-\mathrm{lbf}$ & $\pm 1,000 \mathrm{in}-\mathrm{lbf}$ & 1.00 & $\pm 0.80 \mathrm{in}-\mathrm{lbf}$ \\
\hline Moment about Y-axis & $\pm 80 \mathrm{in}-\mathrm{lbf}$ & $\pm 1,000 \mathrm{in}-\mathrm{lbf}$ & 1.25 & $\pm 1.0 \mathrm{in}-\mathrm{lbf}$ \\
\hline Moment about Z-axis & $\pm 80 \mathrm{in}-\mathrm{lbf}$ & $\pm 1,200 \mathrm{in}-\mathrm{lbf}$ & 1.25 & $\pm 1.0 \mathrm{in}-\mathrm{lbf}$ \\
\hline
\end{tabular}

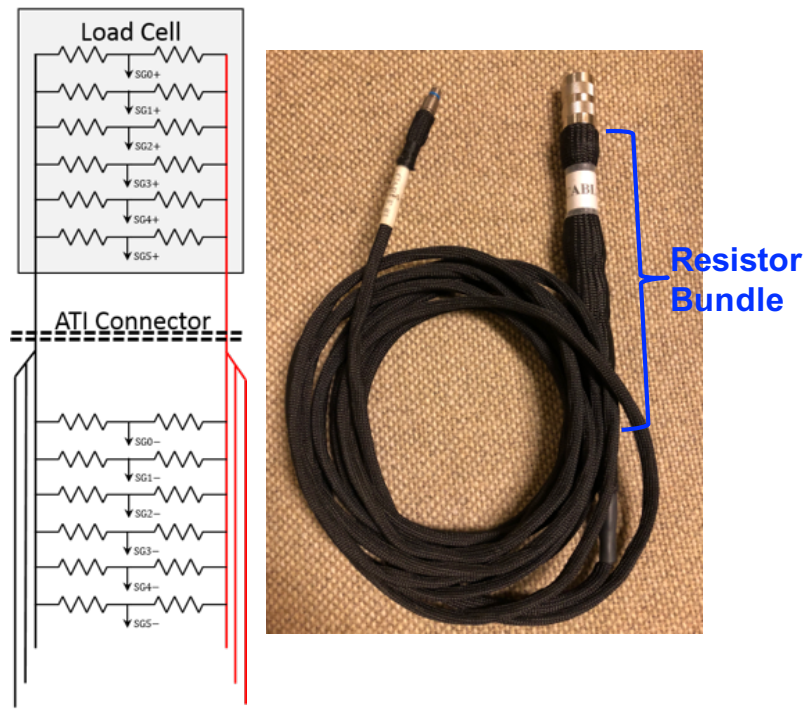

(a) Completion circuit cable

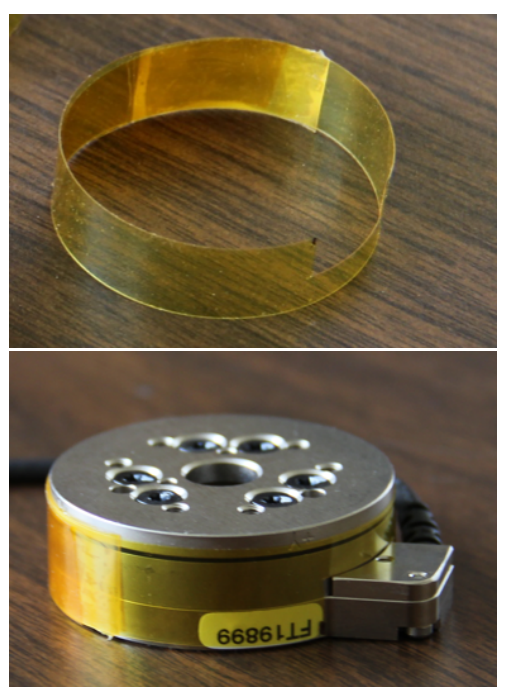

(b) Kapton ring around metric gap

Figure 23. Special implementation considerations for use of the Mini45-ERA load cells in the SRBs.

\section{SRB In situ System Calibration}

Prior to use, an in situ calibration of the integrated SRB force measurement system was performed in the test section of the $14 \times 22$ and was intended to remove any misalignment effects encountered during assembly due to fabrication tolerances. Capitalizing on the single-vector concept used for balance calibration ${ }^{6,7}$ and loading capabilities in the $14 \times 22$ test section, an efficient, cost-effective, and fully-assembled system-level calibration experiment was designed and executed for the SRB FMS. Using a statistical design of experiment calibration strategy, a factorial load schedule was designed that featured 1-, 2-, and 6-component loads as shown in Figure 24(a). Multicomponent loadings were achieved by angular positioning of the model based on the single-vector concept. Because the VST-15 balance was operational during the calibration, precise setpoints were not required since the applied load was measured directly. This reduced the need for a precise load fixture and also increased the efficiency of the loadings as the calibration for both SRBs only took two days.

Figure 24(b) shows photos of the loading hardware used in the calibration. The SRB calibration load block was designed to mount at the SRB balance moment center, which is located at the midpoint between load cells. There were load positions at the BMC and \pm 2 inches offset from the BMC for both normal force and side force loadings. A hoist ring installed into the load block and a simple hook load pan were used to apply the calibrated loads to the SRBs. The ring and hook allowed application of multicomponent loads because the load pan was able to reposition itself to the gravity vector as the model was moved to different attitudes. For side force loads into the model, a hanger pivoting on a ball bearing was used instead. 


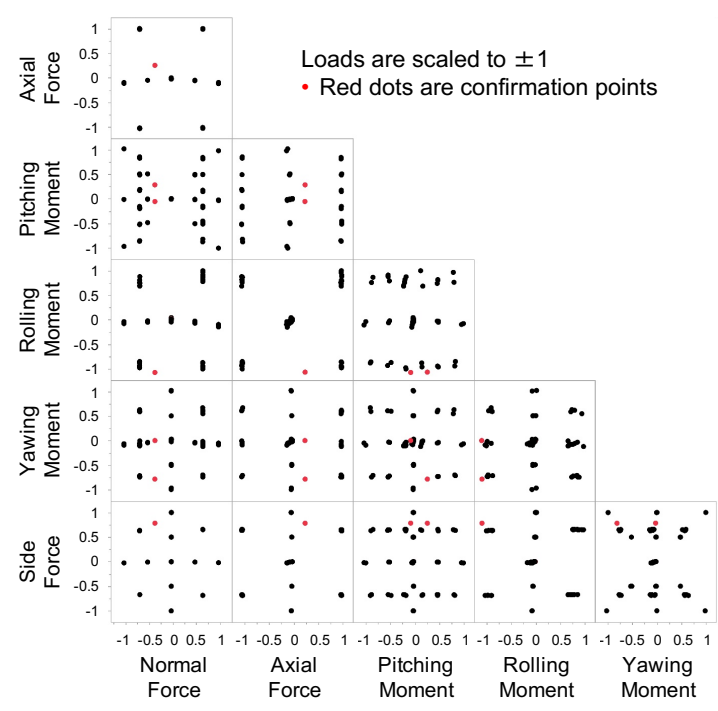

(a) Factorial load schedule

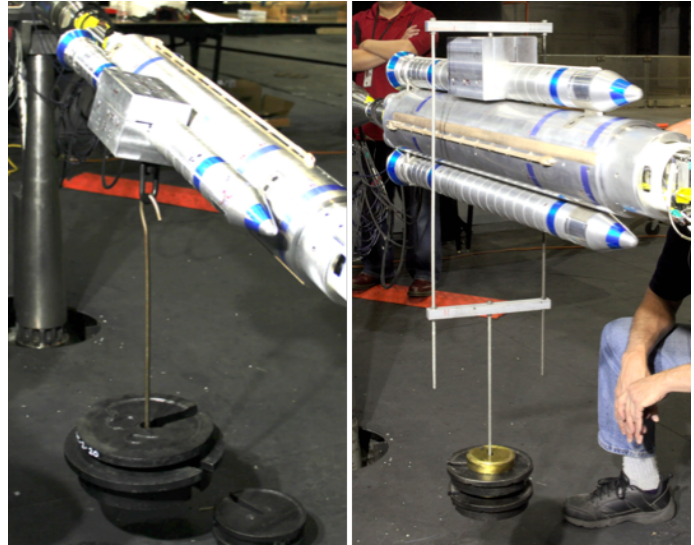

(b) Load fixture hardware

Figure 24. SRB in situ system calibration (a) factorial load schedule, and (b) load fixture hardware.

During the calibration, the outputs from each load cell are converted to F\&M using the factory-provided calibrations and then each pair of load cells in an SRB are combined to the common moment center. The load cells are mounted in the SRBs such that the load cell Z-axis coincides with the SRB Z-axis. However, in the right SRB, the load cell $\mathrm{X}$ and $\mathrm{Y}$ axes are in the opposite orientation from the SRB X and $\mathrm{Y}$ axes (Figure 25). Furthermore, recall that the SRB bodies are on the nonmetric side of the load cells, thus requiring another sign orientation correction to the load cell measurements when applying loads to the SRBs. These sign orientation corrections were all handled in the data reduction.

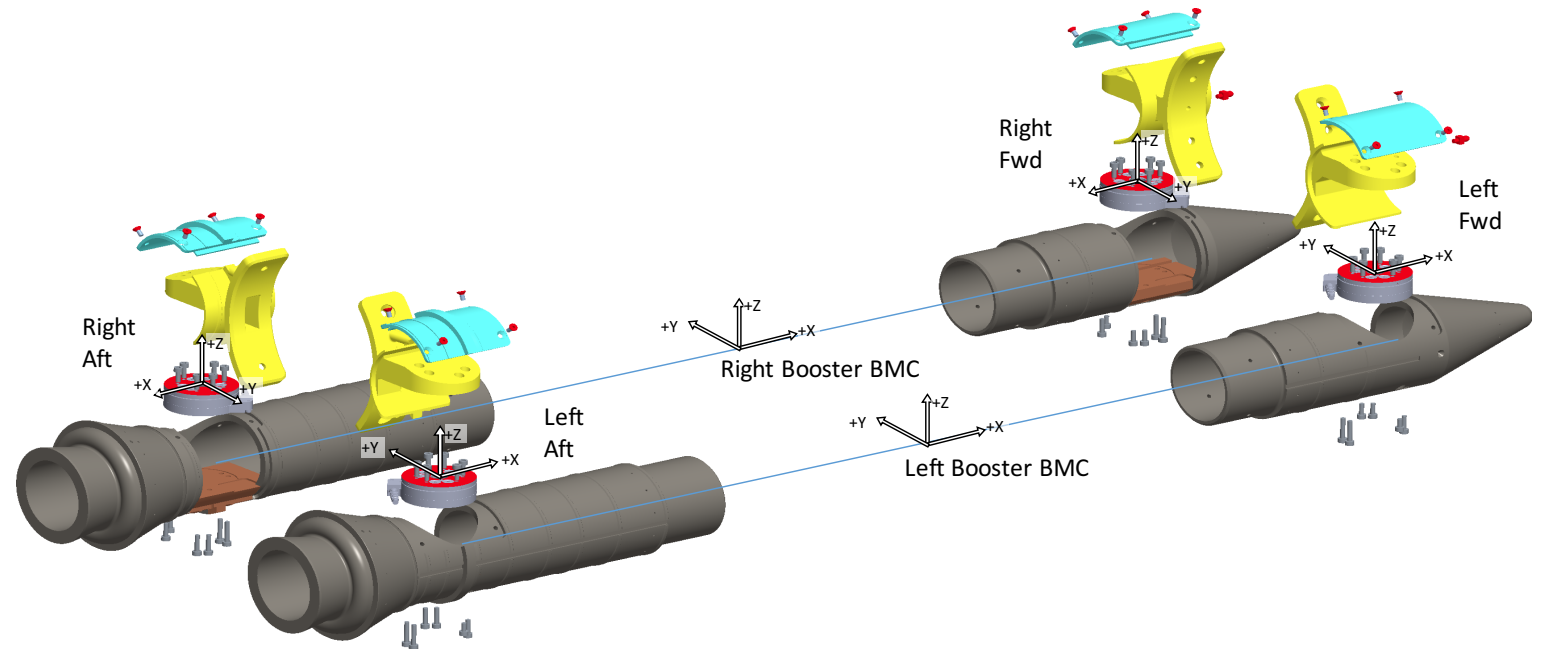

Figure 25. Orientation of the load cells in the right and left SRBs. Note that the diagram is showing the bottom side of the vehicle.

The applied loads measured by the VST-15 balance are also transferred to each SRB moment center. These measurements are then used to determine the SRB calibration model that corrects for assembly misalignments. Results from the in situ system calibration showed significant improvement over the uncorrected misaligned system setup and even showed some improvement over the factory-provided calibration. The reported accuracies, less than 1 percent of full-scale for the forces and 1 to 3 percent of full-scale for the moments, were well within the desired accuracies of the test team. The reported calibration accuracies are based on $2 \sigma$ values of the back-computed corrected loads and are shown in Table 7 for the right and left SRBs. More information about the SRB calibrations can be found in Refs. 8 and 9 . 
Table 7. Right and Left SRB in situ system calibration summary.

\begin{tabular}{|c|c|c|c|c|c|}
\hline SRB Balance & Calibration & \multicolumn{2}{|c|}{ RSRB $2 \sigma$ Calibration Error } & \multicolumn{2}{|c|}{ LSRB $2 \sigma$ Calibration Error } \\
\cline { 3 - 6 } Component & Load Range & \%-Full Scale & Eng. Units & \%-Full Scale & Eng. Units \\
\hline Axial Force & $\pm 13 \mathrm{lbf}$ & 0.69 & $\pm 0.09 \mathrm{lbf}$ & 0.82 & $\pm 0.11 \mathrm{lbf}$ \\
\hline Side Force & $\pm 25 \mathrm{lbf}$ & 0.49 & $\pm 0.12 \mathrm{lbf}$ & 0.38 & $\pm 0.10 \mathrm{lbf}$ \\
\hline Normal Force & $\pm 100 \mathrm{lbf}$ & 0.34 & $\pm 0.34 \mathrm{lbf}$ & 0.21 & $\pm 0.21 \mathrm{lbf}$ \\
\hline Rolling Moment & $\pm 51 \mathrm{in}-\mathrm{lbf}$ & 1.30 & $\pm 0.66 \mathrm{in}-\mathrm{lbf}$ & 1.20 & $\pm 0.61 \mathrm{in}-\mathrm{lbf}$ \\
\hline Pitching Moment & $\pm 200 \mathrm{in}-\mathrm{lbf}$ & 1.16 & $\pm 2.32 \mathrm{in}-\mathrm{lbf}$ & 0.97 & $\pm 1.94 \mathrm{in}-\mathrm{lbf}$ \\
\hline Yawing Moment & $\pm 50 \mathrm{in}-\mathrm{lbf}$ & 3.47 & $\pm 1.74 \mathrm{in}-\mathrm{lbf}$ & 2.71 & $\pm 1.36 \mathrm{in}-\mathrm{lbf}$ \\
\hline
\end{tabular}

\section{B. Model Attitude}

For the transition testing phase, model attitude angles (pitch and roll) were acquired in two different ways. The primary model attitude measurement was based on a calibration of the vertical post pitch strut using a high precision accelerometer. Model pitch angle was then calculated based on the pitch strut angle, the pitch head knuckle angle, misalignment angles of the sting-balance-model that were measured during installation, and the sting-balance deflection angle based on deflection measurements completed before the test. The model roll angle was measured by a potentiometer located in the roll coupler that was calibrated during the installation phase. The primary method is shown in Figure 26(a) and is the traditional and most precise way of knowing the model attitude in a wind tunnel. The secondary model attitude measurement were three separate onboard accelerometers in an orthogonal arrangement (Figure 26(b)) that allowed for a direct measurement of the model attitude angles (pitch and roll) based on an offline calibration procedure. For most of the test envelope, this measurement was very consistent with the primary calculated attitude. However, there are several issues with the method and one of the issues was that model dynamics and vibrations introduce noise in the measurement (also known as sting whip). The other issue was that when the model is at high total angles of attack $\left(\geq 70^{\circ}\right)$, this type of pitch angle measurement based on gravity becomes imprecise, and also does not allow for a measurement of roll angle at a total angle of attack of $90^{\circ}$. For the tower interference testing phase, the model was always at a total angle of attack of $90^{\circ}$ and since the roll coupler is not used in that testing phase, the model roll angle and wind azimuth angle were determined directly from the facility turntable encoder output.

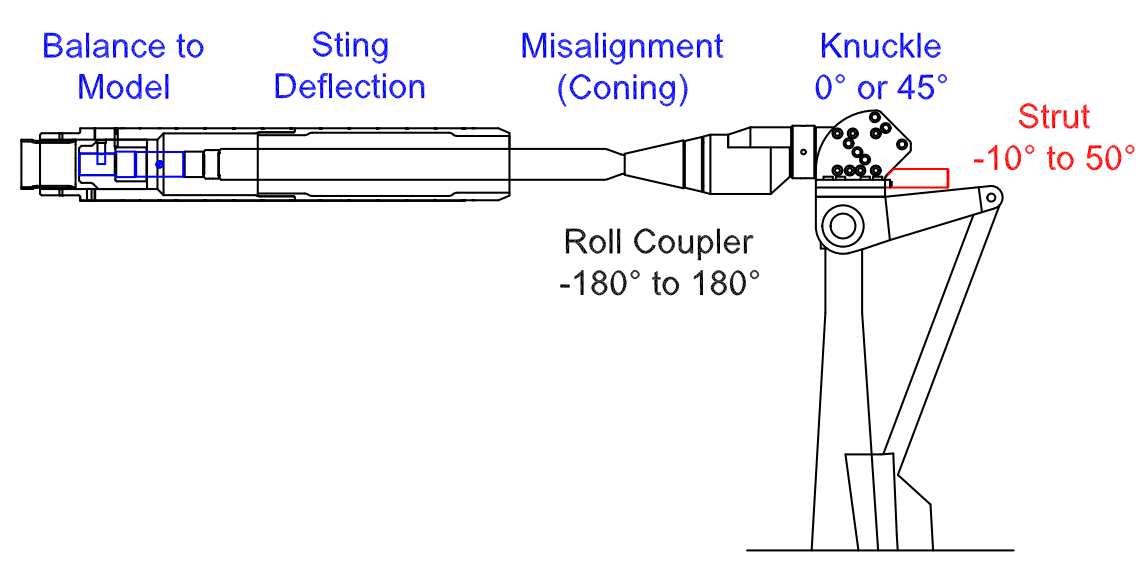

(a) Primary model attitude measurement

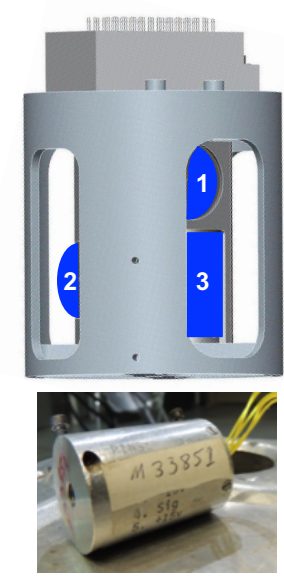

(b) Secondary model attitude measurement

Figure 26. Methods for model attitude measurement for the SLS LOT model : (a) primary and (b) secondary. 


\section{Surface Pressure}

Surface pressures on the core stage were measured using a 64-port 5-psid Electronically Scanned Pressure (ESP) module that was located inside the core stage atop the bracket for the onboard accelerometers (Figure 27 ). The 34 static pressure taps on the lower core stage and the 28 static pressure taps on the three separate upper stages and noses were measured using this ESP module. While there are 48 static pressure taps on each SRB, they were not measured in Test 633 due to space constraints inside the SRBs with the addition of the load cells and accompanying cables. There was simply not enough room to fit an ESP module inside the SRB concurrently with the load cells. The static pressure taps on each SRB were taped over on the model surface to prevent airflow through the taps into the inside of the SRB. The main goal of acquiring a limited set of surface pressures on the vehicle was for CFD validation and comparison. Krist et al. ${ }^{10}$ and Ratnayake et al. ${ }^{11}$ discuss the use of the experimental surface pressures for comparison to the CFD simulations that are used to

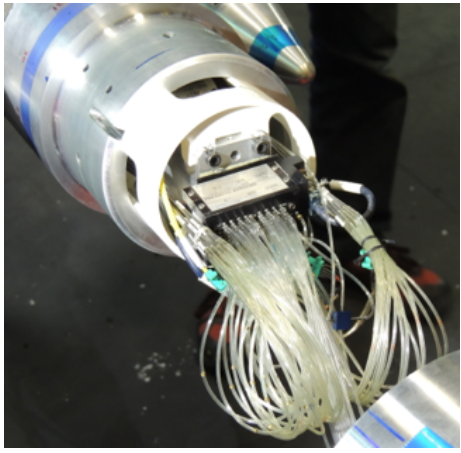

Figure 27. ESP module in core stage used for surface pressure measurements.

produce distributed aerodynamic lineloads for the SLS vehicles in the LOT environment. Therefore, results from the surface pressure measurements will not be discussed in this paper.

\section{Base and Cavity Pressure}

The CSE and SRB plumes were not simulated in the wind tunnel, therefore, the base pressure that is experienced by the vehicle in flight is vastly different than the base pressure measured in the experiment. The flight base pressure aerodynamic database is a separate product that is developed from historical data and very specialized ground tests. The base pressure in the wind tunnel test is therefore measured at the base of the core stage and both SRBs, and a base force is calculated using the base area of each body. This total combined base force from the core and SRBs is then removed from the total axial force measured by the main balance. The base force from each SRB is also removed from the total axial force measured by the SRB load cells. Similarly, due to the off-centerline SRB configuration, a yawing moment could be created from a differential pressure measured on the right and left boosters. A base yawing moment is therefore also calculated and removed. Both of these base corrections result in forebody forces and moments.

Figure 28(a) shows the location of the base pressure measurements for the core stage and the SRBs. The core stage cavity was equipped with four independent base pressure measurements that were averaged to compute the base force on the core stage. Each SRB nozzle cavity was equipped with two independent base pressure tubes (Figure 28(b)) that were also averaged in order to compute the SRB base pressure force and moment. The photo also shows a foam insert that was bonded in place inside the SRB nozzle cavity. This was done to help prevent airflow from intruding inside the SRB and possibly affecting the load cell measurements. While the foam insert is not completely impermeable, it fulfilled the objective as the SRB load cell measurements from the test were overall very good.

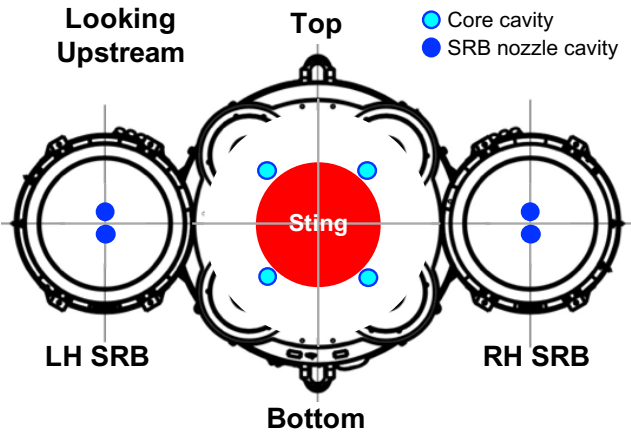

(a) Base pressure measurement
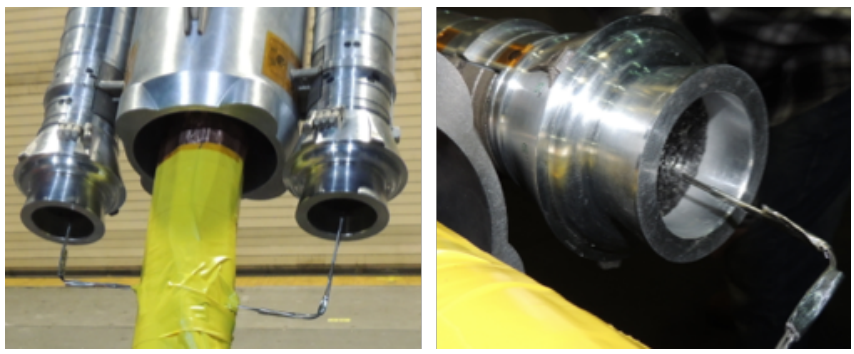

(b) Photos of SRB base pressure tubes including foam insert in SRB nozzle

Figure 28. Base pressure measurements for the SLS LOT model. 


\section{Test Results and Discussion}

\section{A. Data Reduction}

The standard SLS aerodynamic coordinate system (Figure 29) used to post-process wind tunnel data and create aerodynamic databases is the body axis system, in which all forces and moments are tied to the vehicle in all degrees of freedom. The VST-15 balance axis and the SRB load cell axes are essentially coincident with the vehicle body axis, and therefore all F\&M data are acquired in the vehicle body axis. For efficiency in the transition phase of testing, the aerodynamic data are acquired in pitch and roll sweeps, which corresponds to the missile axis system, and therefore, the aerodynamic F\&M data are also transferred to the missile axis system in the data reduction for analysis.

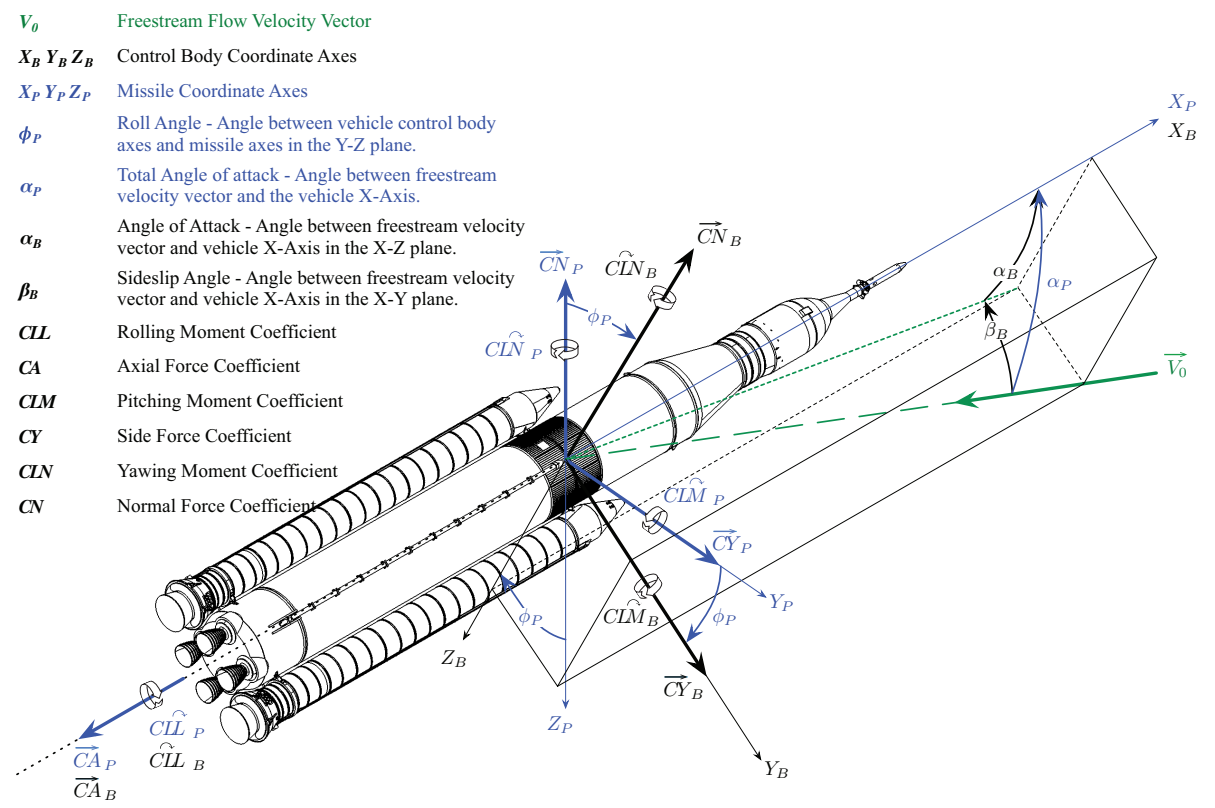

Figure 29. Definition of SLS aerodynamic coordinate system including missile and body axes.

There were a total of five 6-component force transducers used simultaneously in Test 633, where the combination of the two load cells in each SRB essentially acted as a separate six-component balance with the moment center at the midpoint between the two load cells (Figure 30). The F\&M data acquired at each balance moment center were then transferred to the SLS moment reference point (MRP) as required by the test team. The aerodynamic F\&M coefficient data presented in this section are all forebody coefficients meaning that the contribution of the vehicle base areas to the aerodynamic coefficients have been removed through the base pressure correction described in Section VI-D.

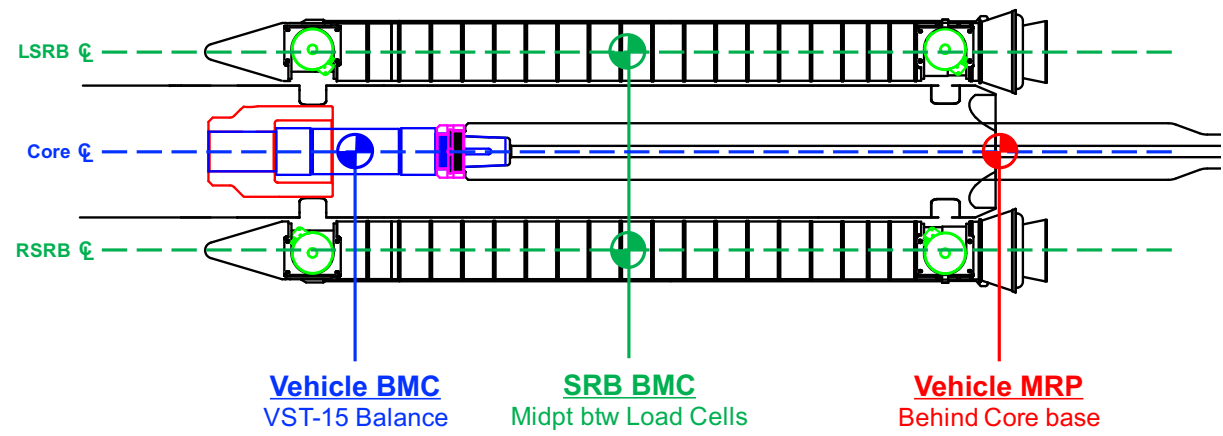

Figure 30. Balance Moment Center \& Moment Reference Point definition for the SLS LOT model. 


\section{B. Transition Aerodynamics}

The aerodynamics of the SLS vehicle configurations during the transition phase testing will not be discussed in detail in this paper. The data from Test 633 were used to update the SLS LOT aerodynamic databases for the three configurations and discussion of the test results along with the database development and uncertainty quantification process are documented in the aerodynamic database substantiation reports. Instead, sample results have been chosen here to either demonstrate the impact of the testing method improvements or to show an interesting flow feature.

Improvements to the stiffness of the sting-balance system for Test 633 were discussed in earlier sections, but the impact of the improvements were readily apparent in the transition phase testing. Figure 31 shows the data parameter space for the transition phase testing of the Block 1B crew configuration as an example in both the missile axis $\left(\alpha_{p}, \phi_{p}\right)$ and the wind axis $(\alpha, \beta)$. Model dynamics in Test 609 prevented data acquisition in a large portion of the total angle-of-attack range between $30^{\circ}$ and $60^{\circ}$ (shown encompassed by dashed lines in the plots), while the stiffer sting-balance system for Test 633 allowed this portion of the test envelope to be acquired for all three SLS configurations.

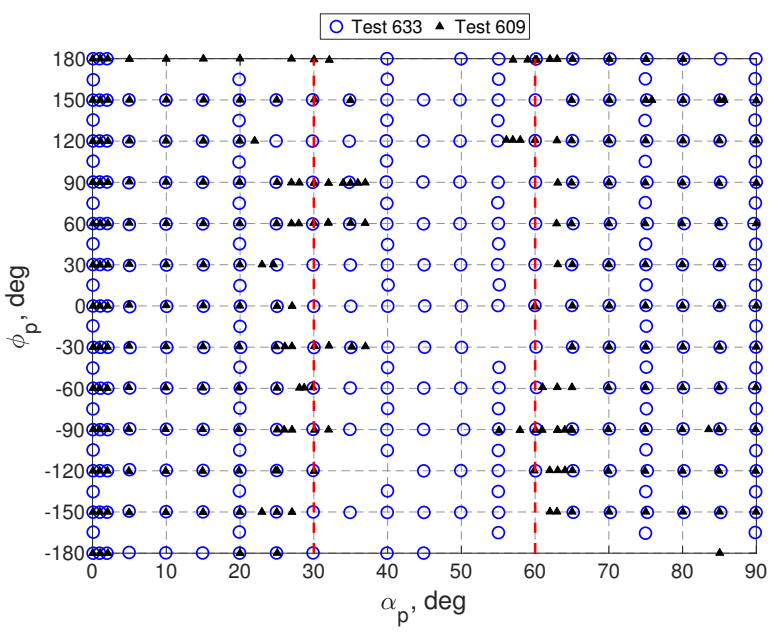

(a) $\alpha_{p}, \phi_{p}$ space

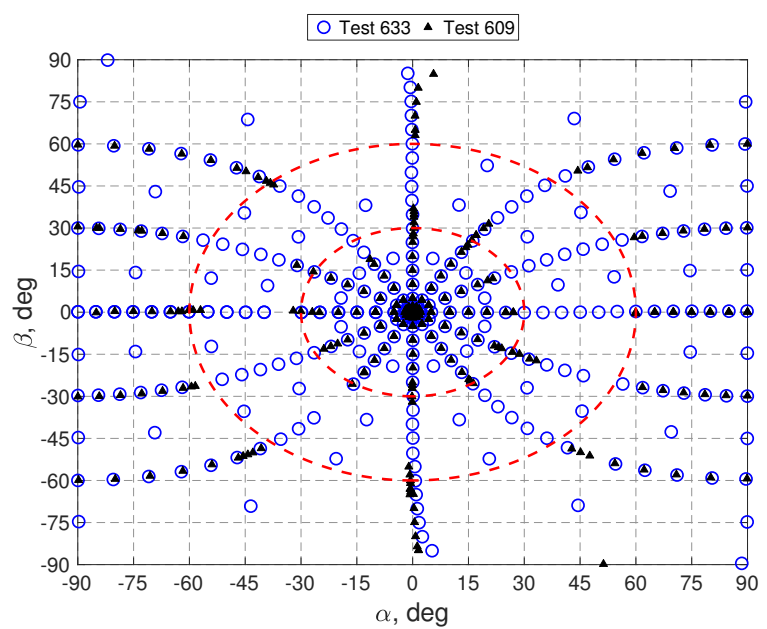

(b) $\alpha, \beta$ space

Figure 31. Comparison of data space between T633 and T609 during transition phase testing for Block 1B crew configuration.

The implementation of the new SRB force measurement system allowed the SRB aerodynamics to be isolated from the full vehicle aerodynamics. Furthermore, the full vehicle and separate SRB aerodynamic data also allowed the core stage aerodynamics to be calculated. These separate data sets allowed insight into how certain flow features affected each vehicle body portion independently. This was also especially helpful for comparisons to CFD simulations of the LOT environment that were used for generation of distributed lineloads for each body. However, since the SRB FMS technique was new, confidence in the quality and validity of the data needed to be gained.

To evaluate the quality and validity of the SRB F\&M aerodynamic data, they are compared to the full stack vehicle aerodynamic data to see if the magnitude and direction of the SRB data make sense. Figure 32 shows a component buildup for the normal force coefficient $(C N)$ and side force coefficient $(C Y)$ at select roll angles for the 28005 configuration. The total full stack vehicle data are shown, as well as contributions from the core stage and the right \& left SRBs. The core stage contribution is calculated by subtracting the RSRB and LSRB data from the full stack vehicle data. At $\phi_{p}=0^{\circ}$, both SRBs are exposed to the freestream flow and thus their contributions to the total vehicle $C N$ should be at a maximum. At this roll angle, each SRB contributes approximately $10-20 \%$ to the total $C N$ depending on the total angle of attack. This is pretty close to the pretest CFD estimates of the SRB contributions. At $\phi_{p}=-90^{\circ}$, the left SRB is on the windward side of the vehicle and the right SRB is on the leeward side, therefore, it is expected that the left SRB will contribute a positive $C Y$ while the right SRB should contribute nearly zero $C Y$ since it is mostly hidden from the freestream flow. The left SRB contributes approximately $10 \%$ to the total $C Y$ at this roll angle. 


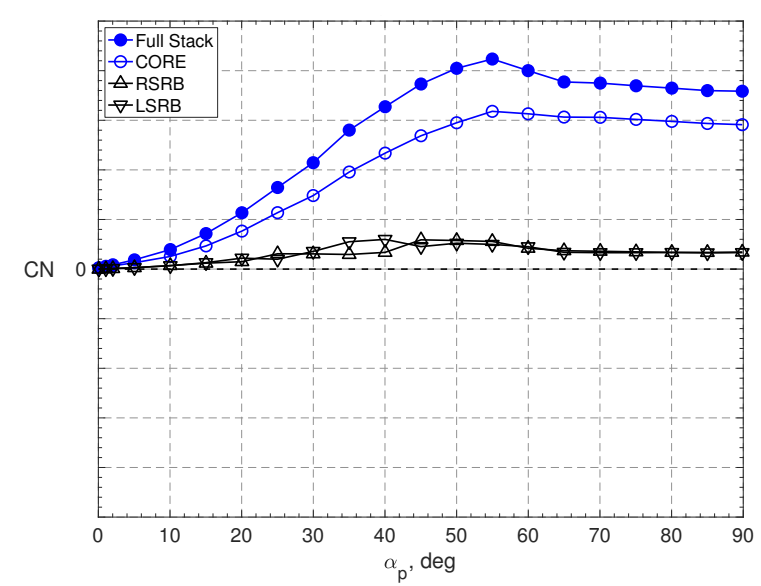

(a) $C N$ at $\phi_{p}=0^{\circ}$

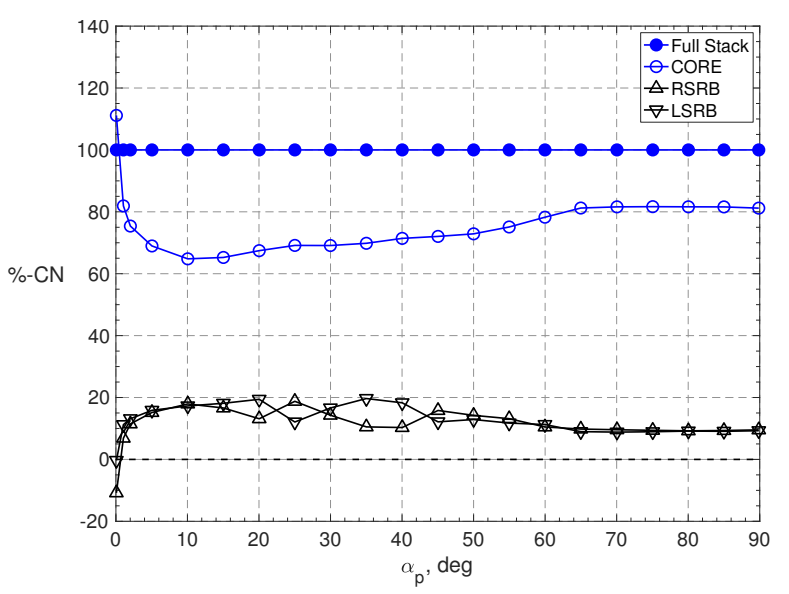

(c) $\%-C N$ at $\phi_{p}=0^{\circ}$

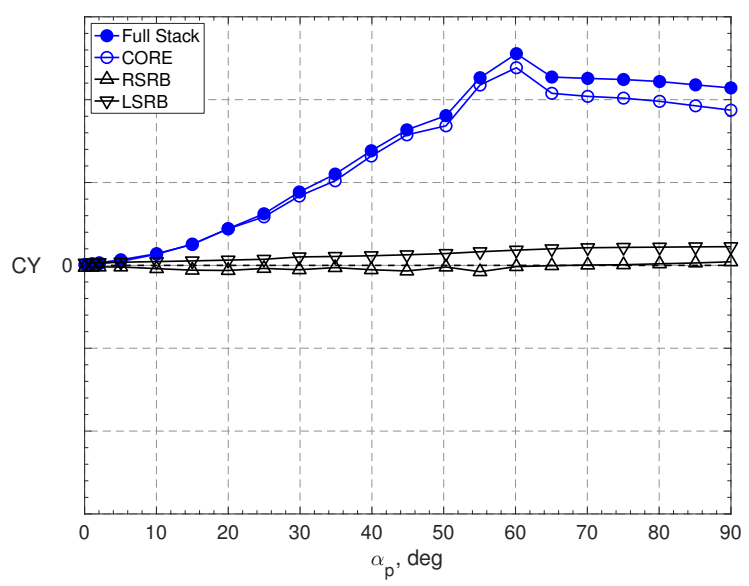

(b) $C Y$ at $\phi_{p}=-90^{\circ}$

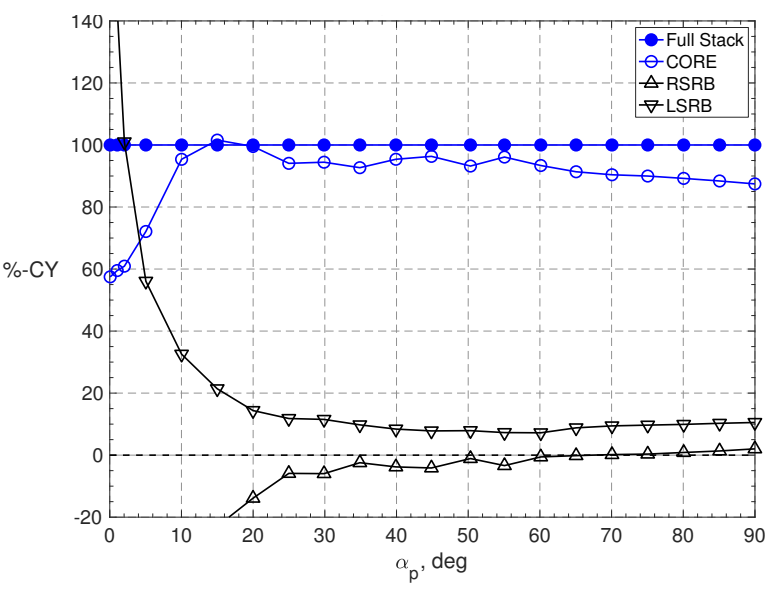

(d) $\%-C Y$ at $\phi_{p}=-90^{\circ}$

Figure 32. Vehicle component contributions to the total $C N$ at $\phi_{p}=0^{\circ}$ and the total $C Y$ at $\phi_{p}=-90^{\circ}$.

Another way to evaluate the validity of the SRB data is to check the symmetry between right and left SRBs. The SLS vehicle has an assumed symmetry plane between the left and right sides of the vehicle as shown in Figure 33(a), although protuberances such as the systems tunnel violate this assumption. However, the aerodynamic F\&M data from the SRBs should be pretty symmetric regardless. Applying the symmetry plane to the missile axis roll angle, $\phi_{p}$, shown in Figure 33(b), produces the equations of symmetry for the SRB aerodynamic coefficient data shown in Equation 1. It is expected that the RSRB longitudinal aerodynamic coefficients at a given roll angle $\phi_{p}$ would be equal to the LSRB coefficients at the opposite roll angle $-\phi_{p}$. It is also expected that the RSRB lateral/directional aerodynamic coefficients at a given roll angle $\phi_{p}$ would be equal in magnitude but opposite in sign to the LSRB coefficients at the opposite roll angle $-\phi_{p}$.

$$
\begin{aligned}
(C A)_{R S R B,+\phi_{p}} & =(C A)_{L S R B,-\phi_{p}} \\
(C N)_{R S R B,+\phi_{p}} & =(C N)_{L S R B,-\phi_{p}} \\
(C L M)_{R S R B,+\phi_{p}} & =(C L M)_{L S R B,-\phi_{p}} \\
(C Y)_{R S R B,+\phi_{p}} & =-(C Y)_{L S R B,-\phi_{p}} \\
(C L L)_{R S R B,+\phi_{p}} & =-(C L L)_{L S R B,-\phi_{p}} \\
(C L N)_{R S R B,+\phi_{p}} & =-(C L N)_{L S R B,-\phi_{p}}
\end{aligned}
$$




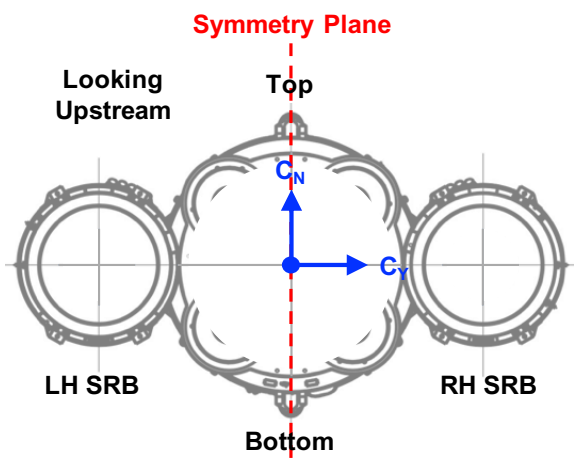

(a) Symmetry plane

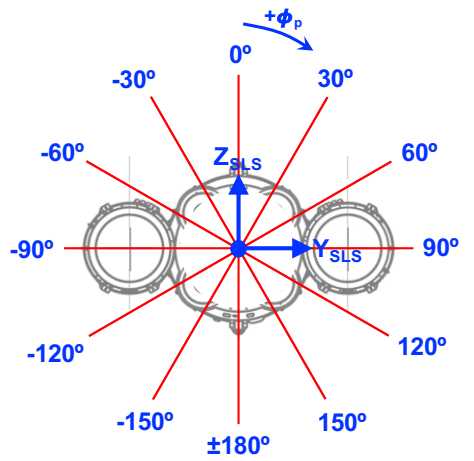

(b) $\phi_{p}$ definition

Figure 33. Left-Right assumed symmetry plane on SLS vehicle.

These symmetry relationships are shown for the SRB data in Figure 34 for $C N_{S R B}$ and $C Y_{S R B}$ at $\phi_{p}= \pm 120^{\circ}$ for the Block $1 \mathrm{~B}$ crew configuration. The plots show the original RSRB data at $\phi_{p}=120^{\circ}$, the LSRB data at the opposite roll angle of $\phi_{p}= \pm-120^{\circ}$, and finally the LSRB data after application of the symmetry equation. It can be seen that after application of the symmetry equations, the RSRB and LSRB data compare very well, thus providing more confidence in the SRB F\&M data.

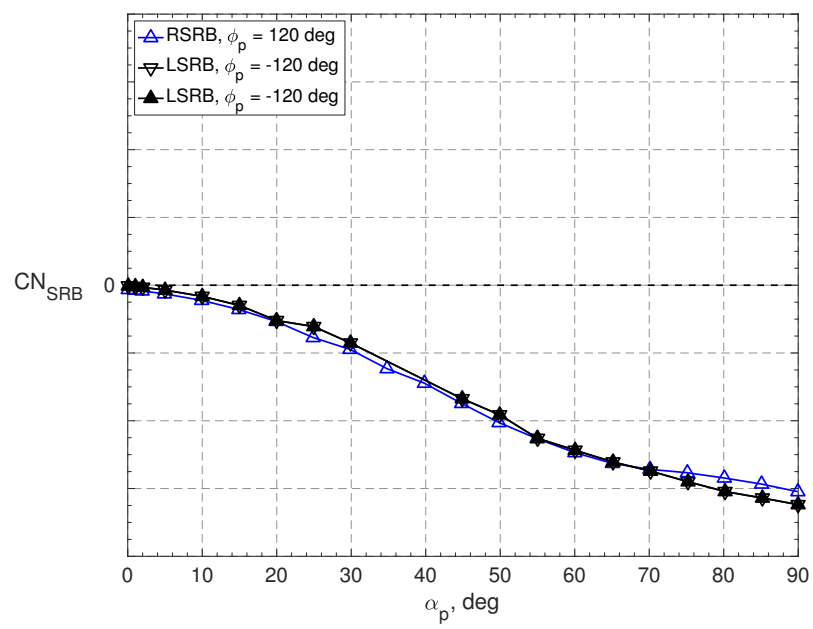

(a) $C N_{S R B}$ at $\phi_{p}= \pm 120^{\circ}$

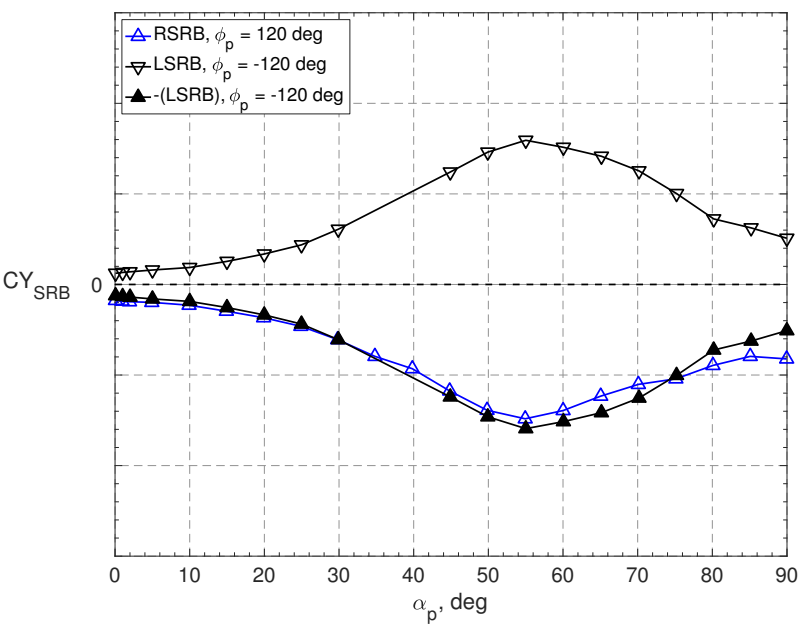

(b) $C Y_{S R B}$ at $\phi_{p}= \pm 120^{\circ}$

Figure 34. SRB data symmetry relations for (a) $C N_{S R B}$ and (b) $C Y_{S R B}$ at $\phi_{p}= \pm 120^{\circ}$.

Since the improvements to the sting-balance system allowed data acquisition in the total angle-of-attack range between $30^{\circ}$ and $60^{\circ}$ and the implementation of the new SRB FMS allowed the aerodynamics of each body to be isolated, some interesting insights into the data in this region were gained. It was noticed that the magnitude of the aerodynamic coefficients in this total angle-of-attack region can vary wildly and significant lateral loading was measured in unexpected areas of the test envelope. Though difficult to predict, it is a well known phenomenon in the missile and slender body aerodynamics community that a steady asymmetric vortex pattern can lock-in between approximately $30^{\circ}$ and $60^{\circ}$ angle of attack. This has been seen in past research on slender body aerodynamics with two examples from Ericsson ${ }^{12}$ and Kubin ${ }^{13}$ as shown in Figure 35. The diagrams illustrate the difference in the vortex shedding process as a function of angle of attack. At very low angles of attack, the flow is attached and no vortex shedding is exhibited, and as the angle of attack is increased to about $25^{\circ}$, any vortex shedding off the body is symmetric. Between $25^{\circ}$ and $50^{\circ}$ angle of attack, the vortex shedding becomes asymmetric in a steady fashion, i.e., the strong vortex remains on the same side, once it is established. From $50^{\circ}$ to $70^{\circ}$ angle of attack, the asymmetric shedding becomes 
unsteady and could start alternating from side to side at a particular frequency. Above $70^{\circ}$ angle of attack, the shedding becomes turbulent, and a turbulent shear layer develops on each side of the slender body. These findings, though applied to a simple slender body of revolution, seem to coincide with the data trends from the SLS LOT test, as the range of angles where the steady asymmetric vortex formation occurred is very consistent with those findings.

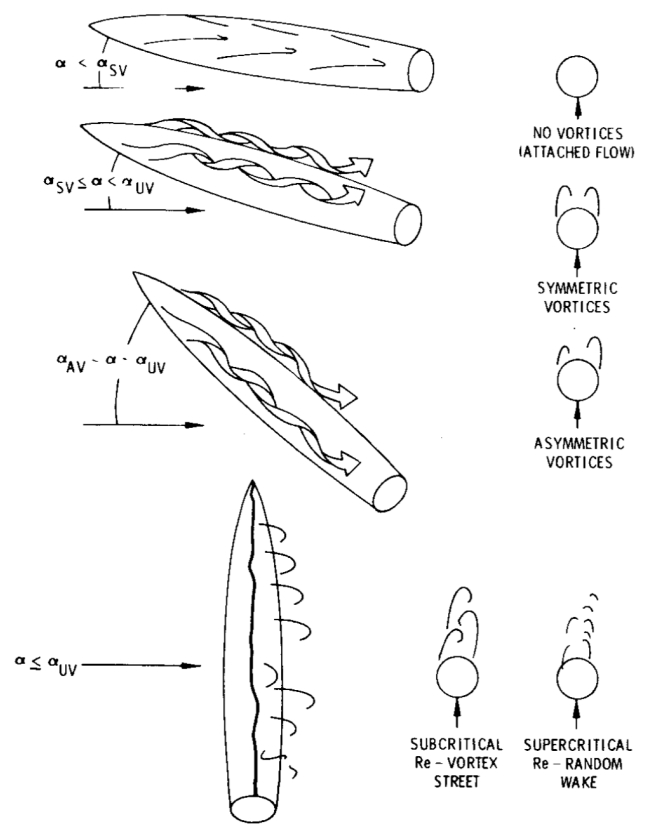

(a) Description by Ericsson ${ }^{12}$
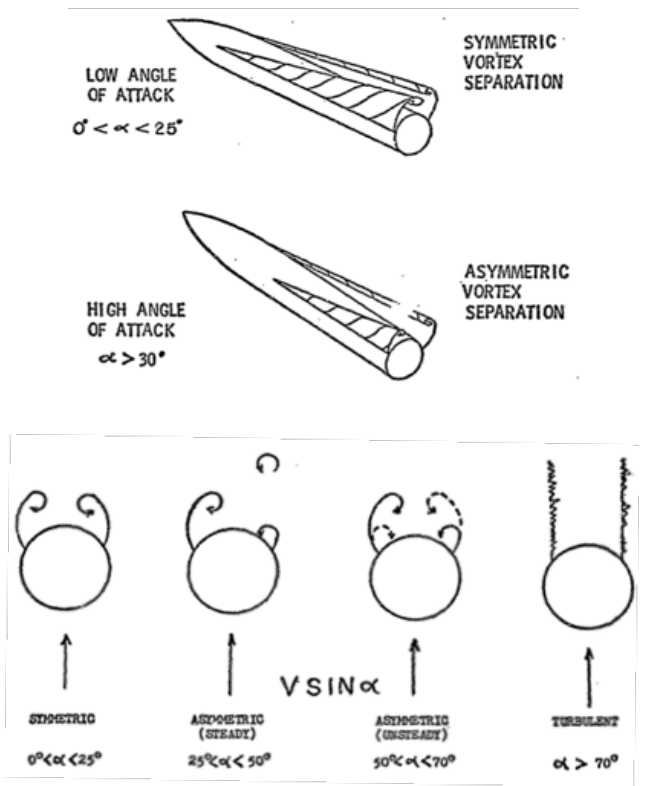

(b) Description by Kubin ${ }^{13}$

Figure 35. Diagrams describing the asymmetric vortex shedding at high angles of attack for slender bodies.

Figure 36 shows evidence in the SLS transition data of large unexpected lateral loading due to asymmetric vortices for the Block 1B crew and cargo configurations. The lateral side force coefficient in the missile axis is shown for the 28005 and 27005 configurations at $\phi_{p}=0^{\circ}, 180^{\circ}$ and $\phi_{p}= \pm 90^{\circ}$. The total contributions from the SRBs (RSRB + LSRB) and the contribution from the core stage are shown in the plots. It is expected that the lateral side force in the missile axis should be near zero at roll angles where the vehicle is symmetric in the $\mathrm{X}-\mathrm{Z}$ plane (left-right). This is largely true for the lower total angles of attack, however, once the vehicle reaches the total angle-of-attack region between $20^{\circ}$ and $70^{\circ}$, the data show that there is unexpected large lateral loading on the vehicle. The lateral loading seems to be largely concentrated on the core stage since the total SRB data are relatively near zero for the entire total angle of attack range. For the 28005 crew vehicle, the lateral loading on the core stage flips back and forth from one side to the other as the total angle-of-attack is increased, possibly indicative of unsteady asymmetric vortices. For the 27005 cargo vehicle, the lateral loading on the core stage tends to stay on one side in this midrange total angle-ofattack region, but can change sides based on the roll angle. This may be indicative of steady asymmetric vortices that set up a strong vortex on one side due to a geometry feature, but then flips to the other side as that geometry feature moves with roll angle. Because the data in this region are so unpredictable, the aerodynamic database was delivered where the lateral loading on either side is equally likely and the GN\&C simulations randomly choose which aerodynamic data to use. 


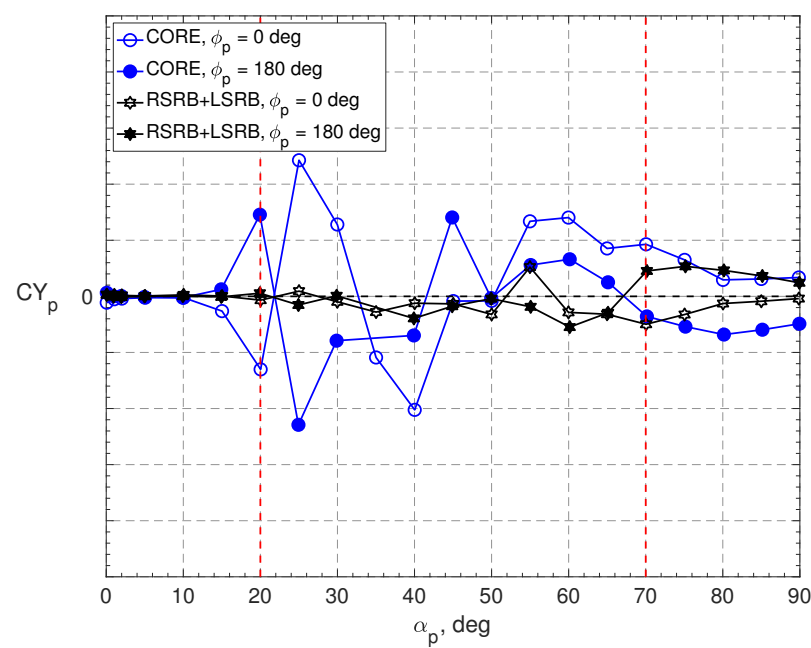

(a) $C Y_{p}$ at $\phi_{p}=0^{\circ}, 180^{\circ}$ for 28005 vehicle

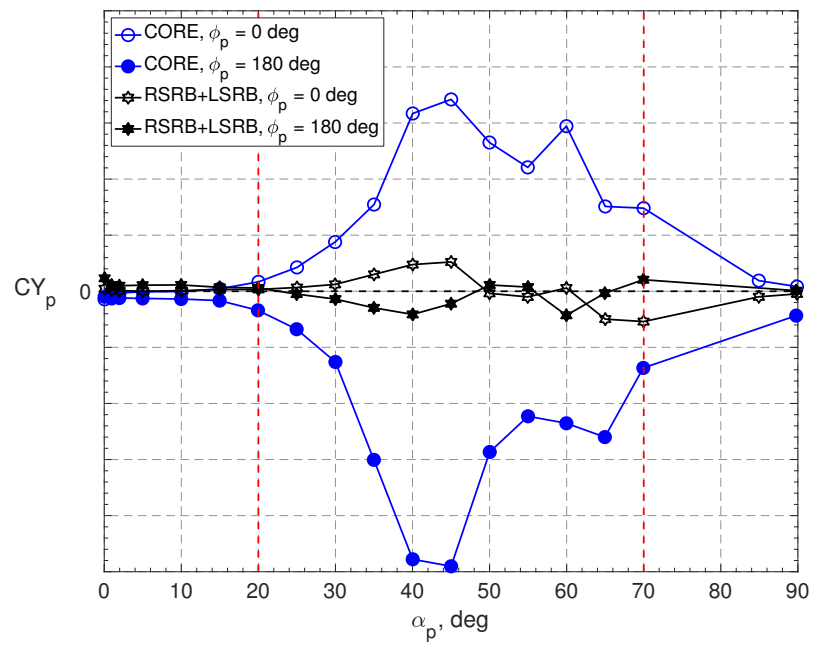

(c) $C Y_{p}$ at $\phi_{p}=0^{\circ}, 180^{\circ}$ for 27005 vehicle

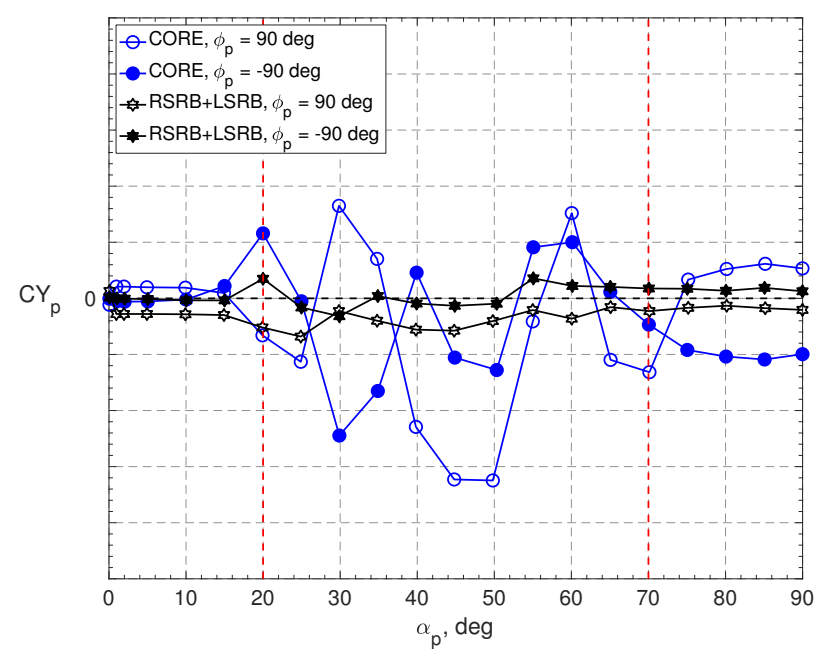

(b) $C Y_{p}$ at $\phi_{p}= \pm 90^{\circ}$ for 28005 vehicle

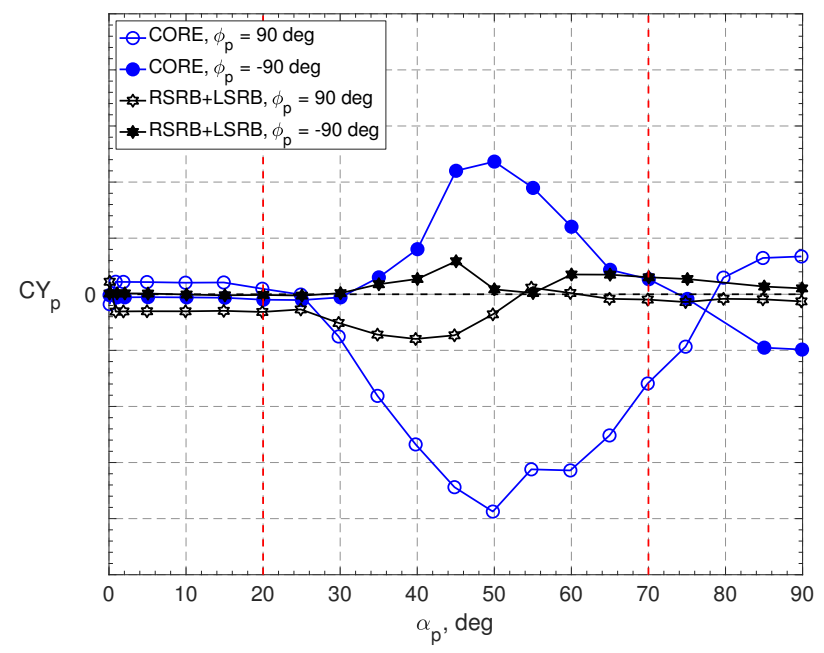

(d) $C Y_{p}$ at $\phi_{p}= \pm 90^{\circ}$ for 27005 vehicle

Figure 36. Lateral loading due to asymmetric vortices for 28005 and 27005 vehicle configurations at $\phi_{p}=0^{\circ}, 180^{\circ}$ and $\phi_{p}= \pm 90^{\circ}$.

\section{Liftoff Tower Interference Aerodynamics}

Similar to the transition phase testing, the tower interference aerodynamics will not be discussed in detail in this paper, but sample results are shown to provide insight into the tower interference effects. First, the improvements to the sting-balance system helped in minimizing the model dynamics during the tower interference testing and data were able to be acquired around wind azimuth angles of $30^{\circ}$ and $330^{\circ}$ where dynamics prevented data acquisition in Test 609 as shown in Figure 37. Also, the new top-half tower allowed data acquisition with the vehicle above the top of the tower $(h / L>1.0)$ to capture when the tower interference effect diminishes to zero.

Recall that in order to determine tower interference increments in the test, the vehicle is first tested without the tower and then it is tested with the full tower and top-half tower. Wind azimuth sweeps at various heights are performed for each tower setup. The tower interference effects can then be seen when looking at the data sets from the two tower setups. Figure 38 shows full vehicle $C N$ and $C Y$ data from wind azimuth sweeps at $h / L=0$ (vehicle on launch pad at liftoff), $h / L=0.5$ (SRB nozzles have reached halfway up the tower at liftoff), and $h / L=1.0$ (SRB nozzles clearing the top of the tower) for the 28005 configuration. In the plots, the data without the tower are shown along with data that have the full tower or top-half tower in proximity. At $h / L=0$, the tower has an effect on $C N$ for nearly every wind azimuth angle, 


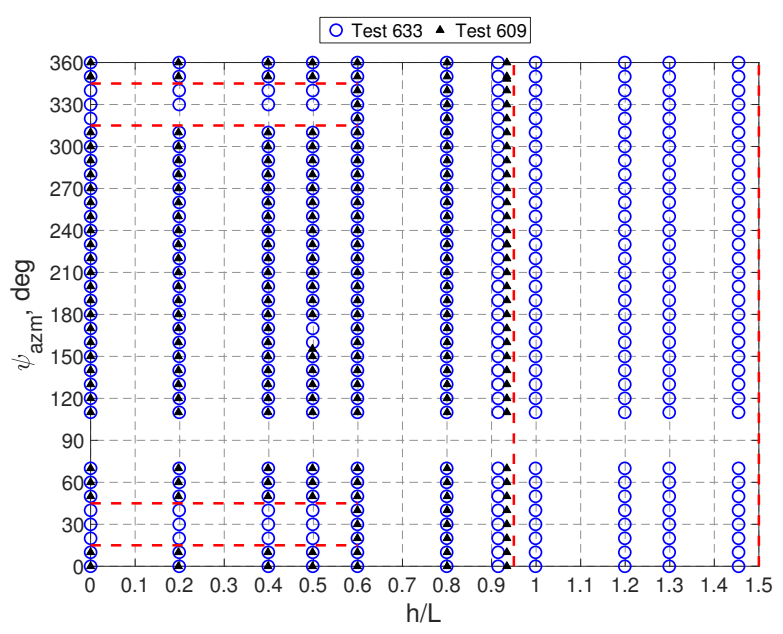

Figure 37. Comparison of $h / L, \psi_{a z m}$ data space between T633 and T609 during tower interference testing for Block 1B crew configuration.

with the largest differences between no-tower and tower data being for northerly winds $\left(\psi_{a z m}=330^{\circ}\right.$ to $\left.30^{\circ}\right)$. This is to be expected because the presence of the tower shields the vehicle from the oncoming flow and thus leads to a less positive $C N$. For southerly winds $\left(\psi_{a z m}=150^{\circ}\right.$ to $\left.210^{\circ}\right)$, the presence of the tail service mast umbilicals (TSMU) on the launch pad floor blocks some of the oncoming flow and leads to a less negative $C N$ meaning the vehicle doesn't get pushed as much toward the tower. It is interesting that for westerly winds $\left(\psi_{a z m}=250^{\circ}\right.$ to $\left.290^{\circ}\right)$, there is also an effect on $C N$. This is because there is a venturi effect where the flow accelerates as it passes in the channel between the tower and vehicle. This is also why the magnitude of $C Y$ increases for westerly winds. At $h / L=0.5$, the tower interference effects decrease as more of the vehicle is exposed to the freestream flow above the top of the tower. The effect of the TSMU for the southerly winds has gone away as well. Finally, at $h / L=1$, the tower interference effects have mostly gone to zero and the data with the tower match pretty closely to the data without the tower.

Figure 39 shows the tower interference effects as a function of $h / L$ for wind azimuths of $0^{\circ}$ and $240^{\circ}$. Similar trends hold for the north wind as the tower interference effect on $C N$ is largest at $h / L=0$, then slowly decreases with increasing $h / L$ until it has diminished by the time the vehicle reaches $h / L=1$. The same is true for the tower interference effect on $C Y$ for a westerly wind, as the effect is largest at $h / L=0$ and slowly decreases with increasing $h / L$. However, the effect doesn't completely diminish until about $h / L=1.2$.

The symmetry of the SRB F\&M data could also be checked in the tower interference testing phase. Recall that in the tower interference testing setup, the wind azimuth angle coincides with the vehicle roll angle. Therefore, a wind azimuth sweep is basically a roll angle sweep on the vehicle at a total angle of attack of $90^{\circ}$ and the symmetry equations shown earlier still hold. The presence of the tower can complicate things, but the tower is also nearly symmetric (left-right) in the X-Z plane with the elevator shaft being the main component that violates the symmetry. Figure 40 shows the symmetry relations for the $C N_{S R B}$ and $C Y_{S R B}$ data at $h / L=0.5$ with and without the tower. The plots show the original RSRB and LSRB data from the wind azimuth sweep, then the LSRB data after application of the symmetry relations where the data are mirrored across $\psi_{a z m}=180^{\circ}$. It can be seen that after application of the symmetry equations, the RSRB and LSRB data compare well even in the presence of the tower. These symmetry relations are also how the data for the easterly winds (missing due to turntable limitations) were filled in during the database development process. 


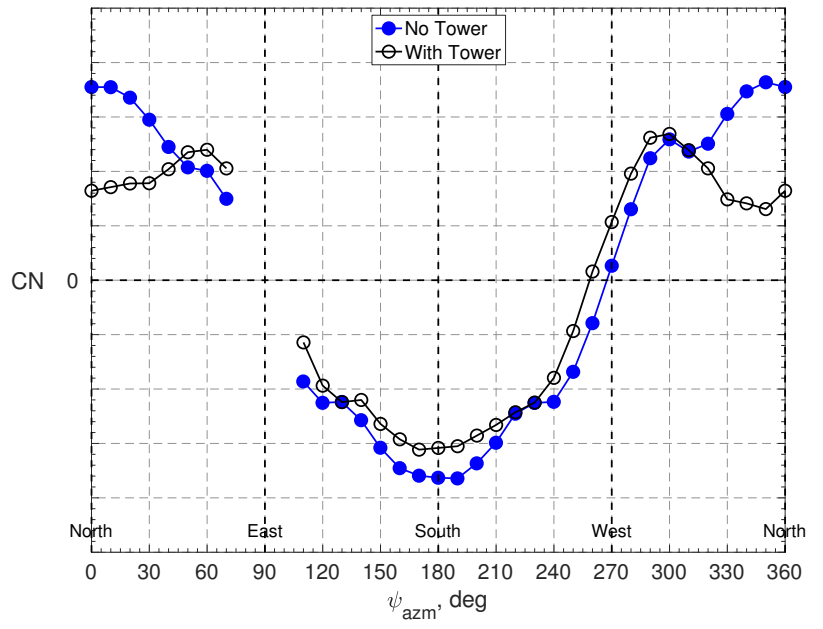

(a) $h / L=0$

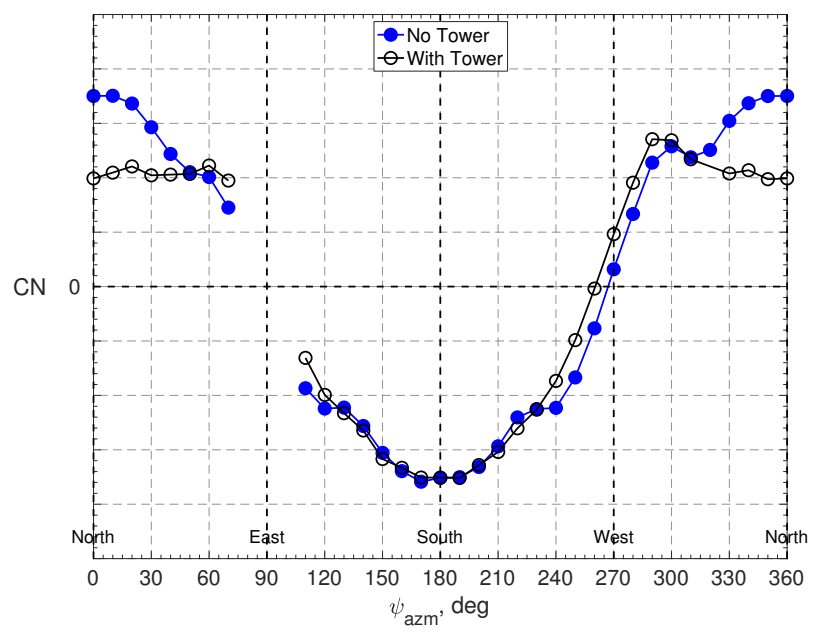

(c) $h / L=0.5$

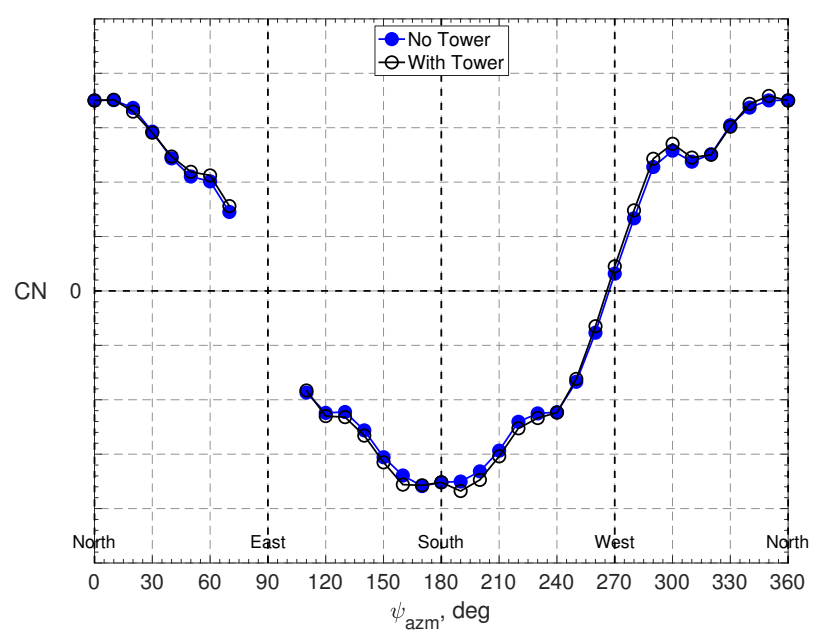

(e) $h / L=1.0$

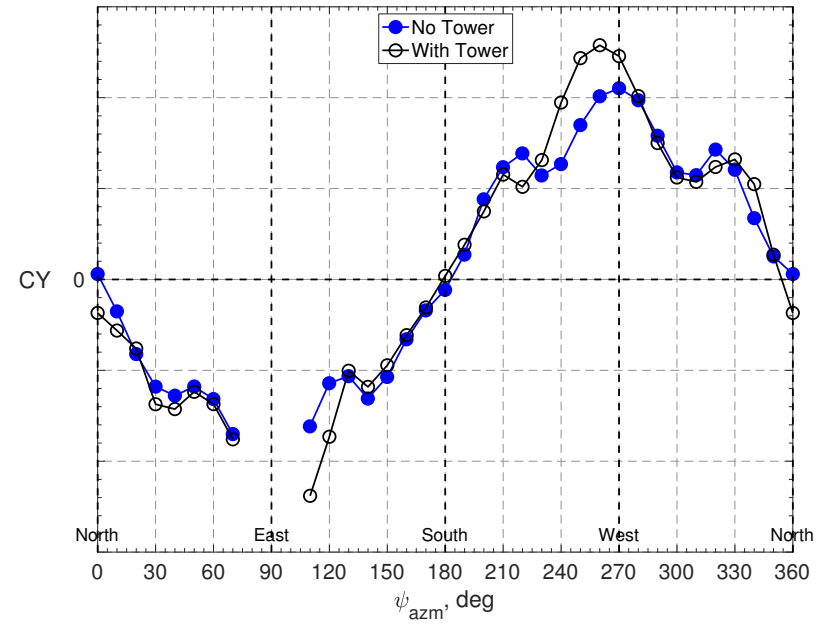

(b) $h / L=0$

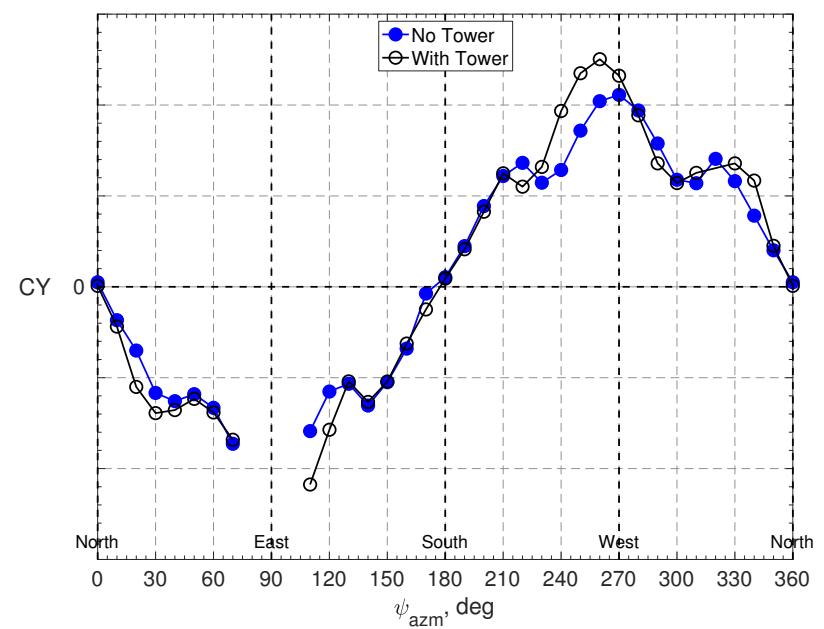

(d) $h / L=0.5$

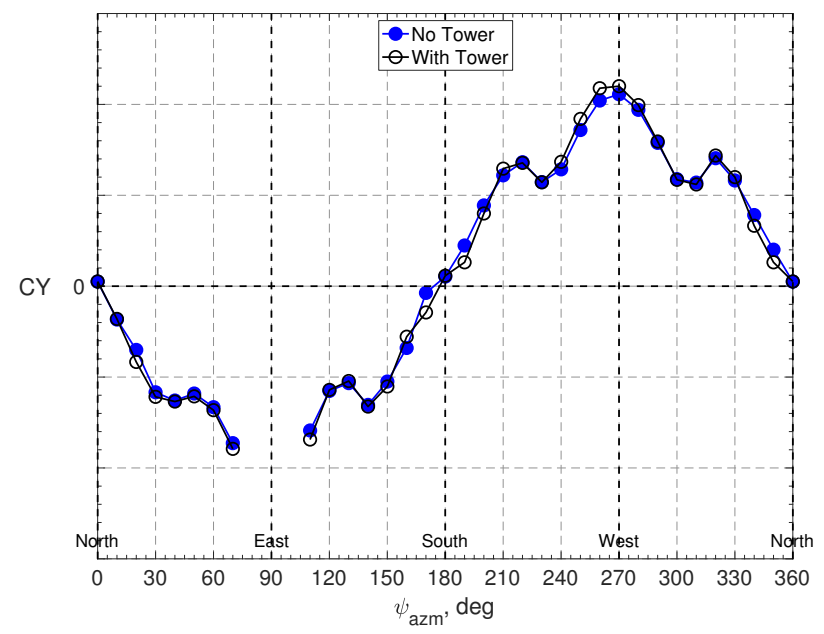

(f) $h / L=1.0$

Figure 38. Tower interference effects for $\mathbf{2 8 0 0 5}$ configuration as a function of $\psi_{a z m}$ at $h / L=0,0.5$, and 1.0 . 


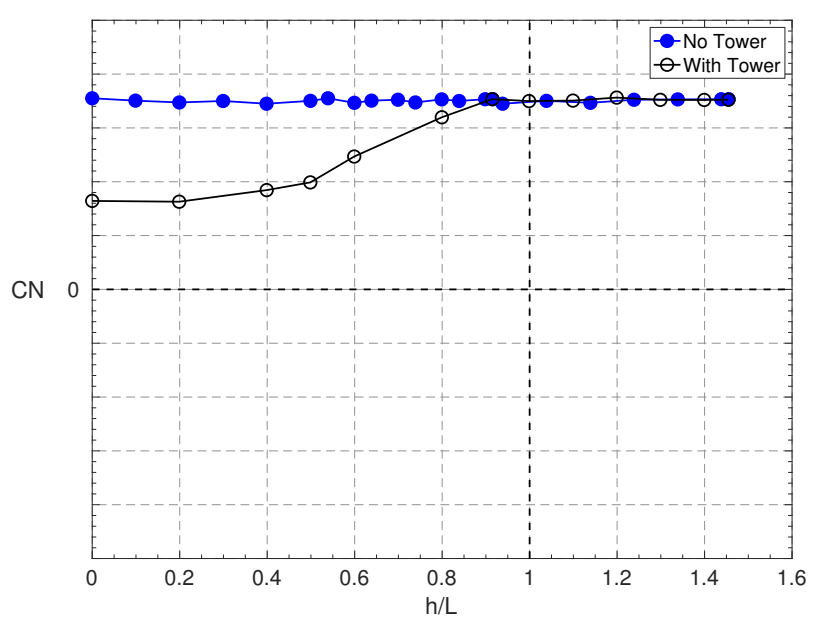

(a) $\psi_{a z m}=0^{\circ}$

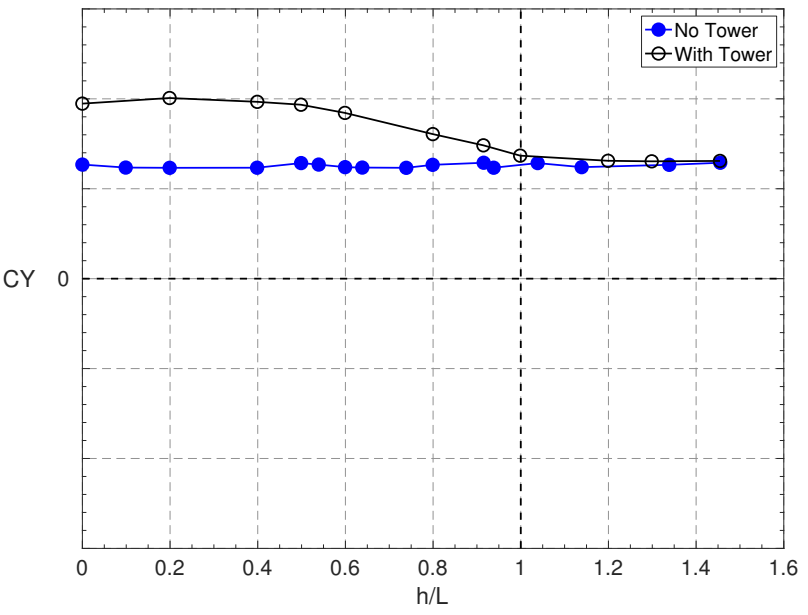

(b) $\psi_{a z m}=240^{\circ}$

Figure 39. Tower interference effects for 28005 configuration as a function of $h / L$ at $\psi_{a z m}=0^{\circ}$ and $240^{\circ}$.

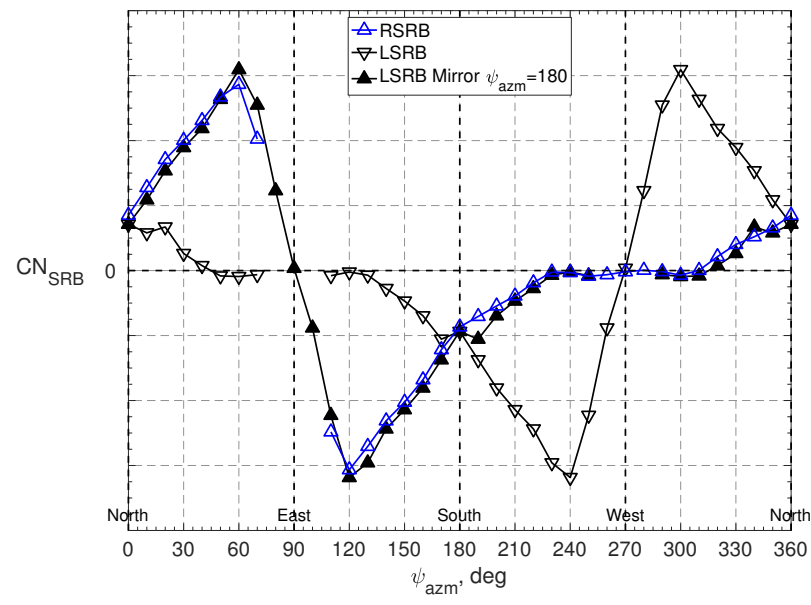

(a) $C N_{S R B}$ at $h / L=0.5$, No Tower

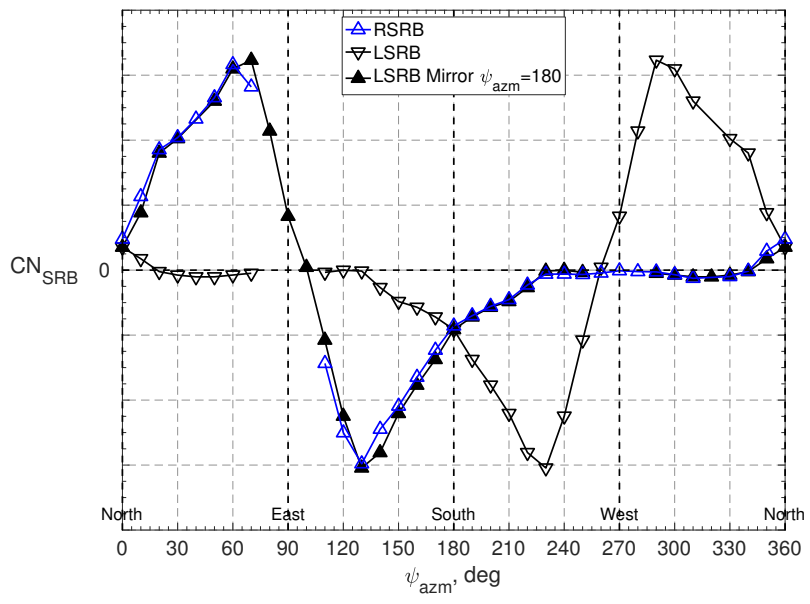

(c) $C N_{S R B}$ at $h / L=0.5$, With Tower

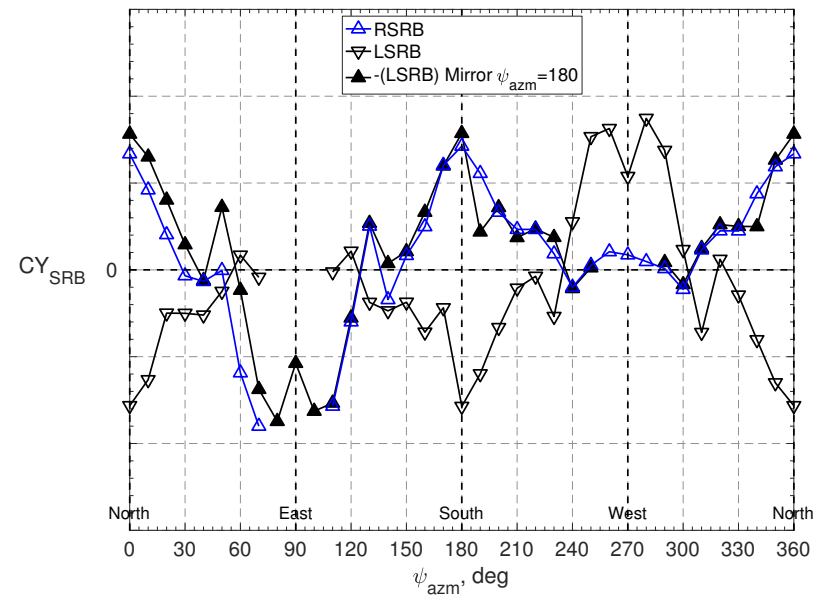

(b) $C Y_{S R B}$ at $h / L=0.5$, No Tower

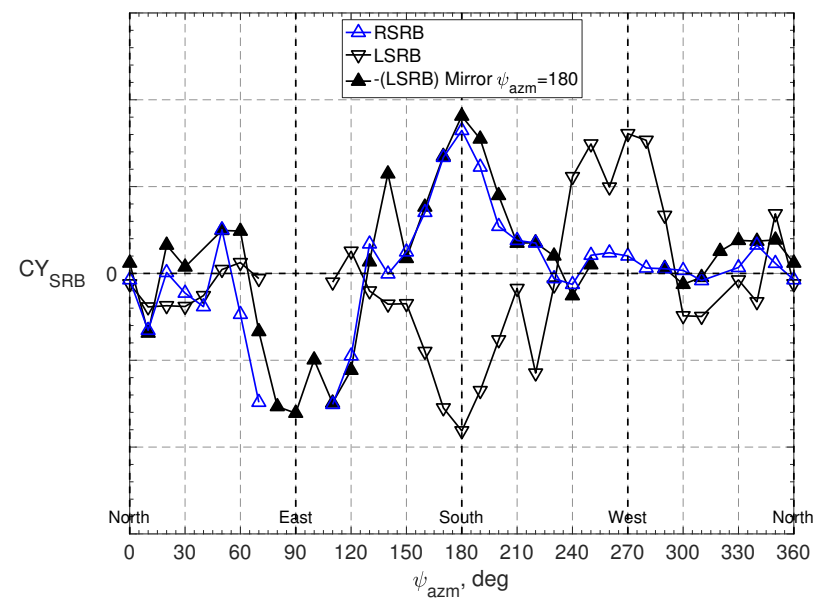

(d) $C Y_{S R B}$ at $h / L=0.5$, With Tower

Figure 40. SRB data symmetry relations for $C N_{S R B}$ and $C Y_{S R B}$ at $h / L=0.5$ with and without tower. 


\section{Smoke Flow Visualization}

During Test 633, smoke flow visualization sessions were performed on the 28005 configuration during the transition phase testing and the tower interference testing with both the full tower and the top-half tower. The improved smoke flow wand and smoke generator machine allowed the test team to see interesting flow features in each phase of testing. The tunnel was run at a low dynamic pressure of 3 psf during these sessions so that it was safe for a smoke wand operator to be in the test section. While this condition is not representative of the main 50 psf test condition, most of the flow physics should still be the same.

During the transition phase testing in the midrange total angles of attack, it was noticed that the flow would wrap around the outside of an SRB and then up and over the top of the vehicle as shown in Figure 41(a). The action was very repeatable and would happen on both SRBs. Also, the smoke flow visualization was able to capture asymmetric vortex shedding off the core stage as shown in Figure 41(b), although it was difficult to determine if the vortices were steady or unsteady. This was much harder to capture as it was difficult to hold the smoke wand steady enough for the smoke to consistently go through the vortices.
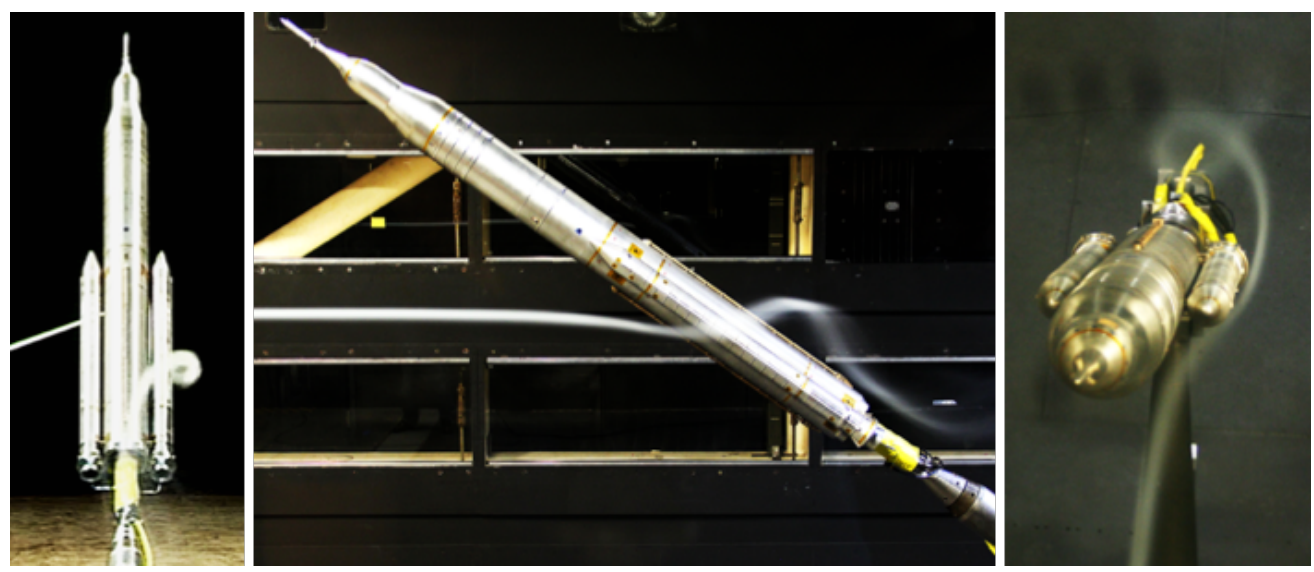

(a) Wrap around SRB
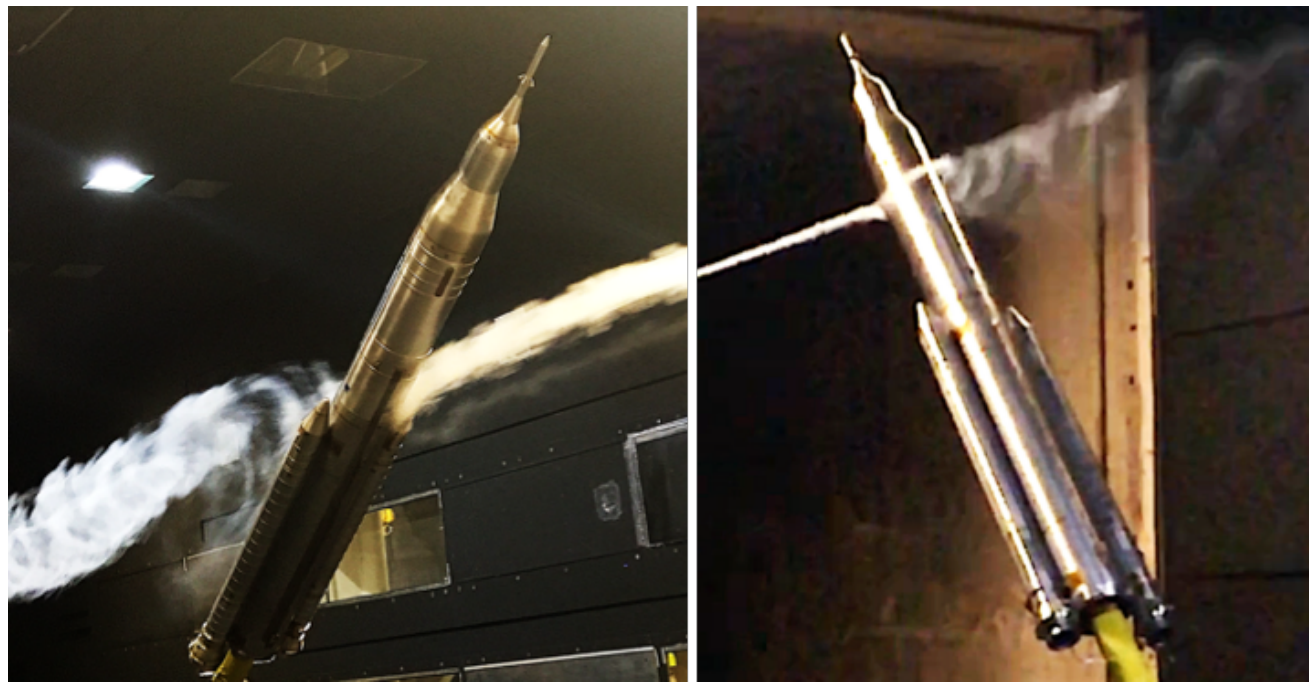

(b) Vortex shedding

Figure 41. Sample smoke flow visualization photos showing (a) flow wrapping around SRB and (b) vortex shedding.

In the tower interference phase of testing, other interesting flow phenomenon also occurred. It was noticed that when the SRB nozzles of the vehicle were near the top of the tower $(h / L$ close to 1$)$, the flow would go over the top of the tower and then get sucked down towards the base of the vehicle as shown in Figure $42(\mathrm{a})$. This would persist until the SRB nozzles of the vehicle were past the top of the tower by about $10 \%$ 
$(h / L=1.1)$. Furthermore, at wind azimuth angles around $30^{\circ}$ and $330^{\circ}$, the flow would perform an S-turn as it snaked around the tower, then between the tower and vehicle, and finally around the vehicle as shown in Figure 42(b). Interestingly, these are also the wind azimuth angles that caused model dynamics in Test 609 suggesting that this was an unsteady event.

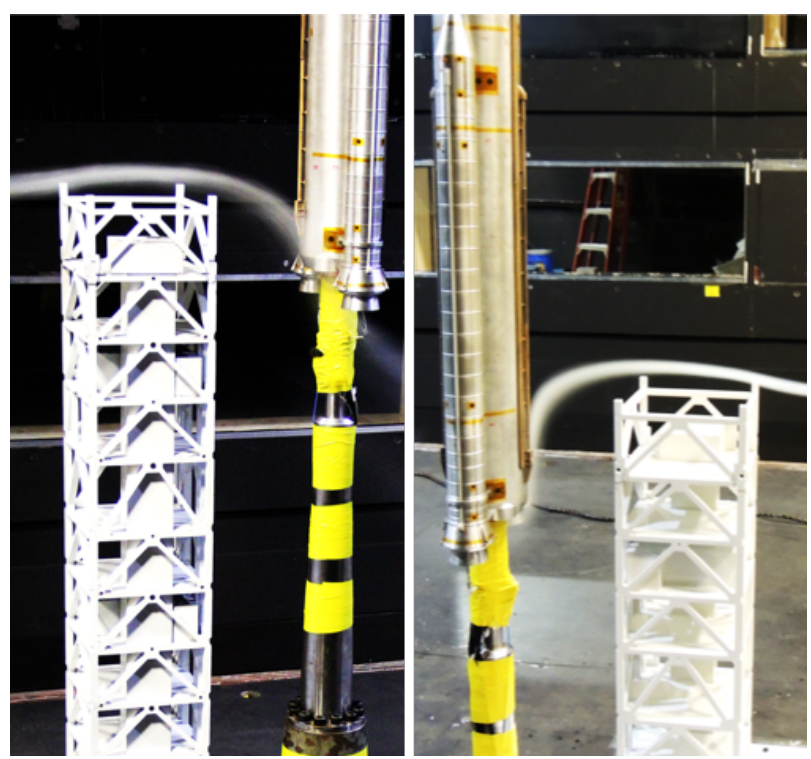

(a) Top of tower

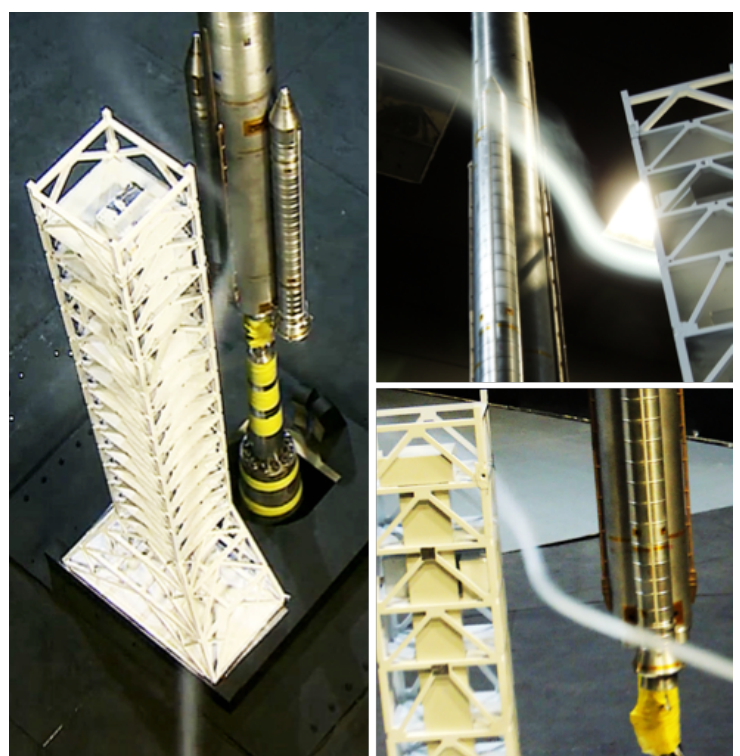

(b) Between tower and vehicle

Figure 42. Sample smoke flow visualization photos showing (a) flow over top of tower and (b) flow between tower and vehicle.

Finally, the smoke flow visualization was able to capture the Coandă effect on the core body and also in the channel between the core and the SRBs. The flow would stick to the curved surface of the core body as it went around the body or in the channel between the core and SRBs as shown in Figure 43. This was also an unsteady event that was difficult to capture during the smoke flow sessions, but it showed that the flow can go between the core and SRBs.
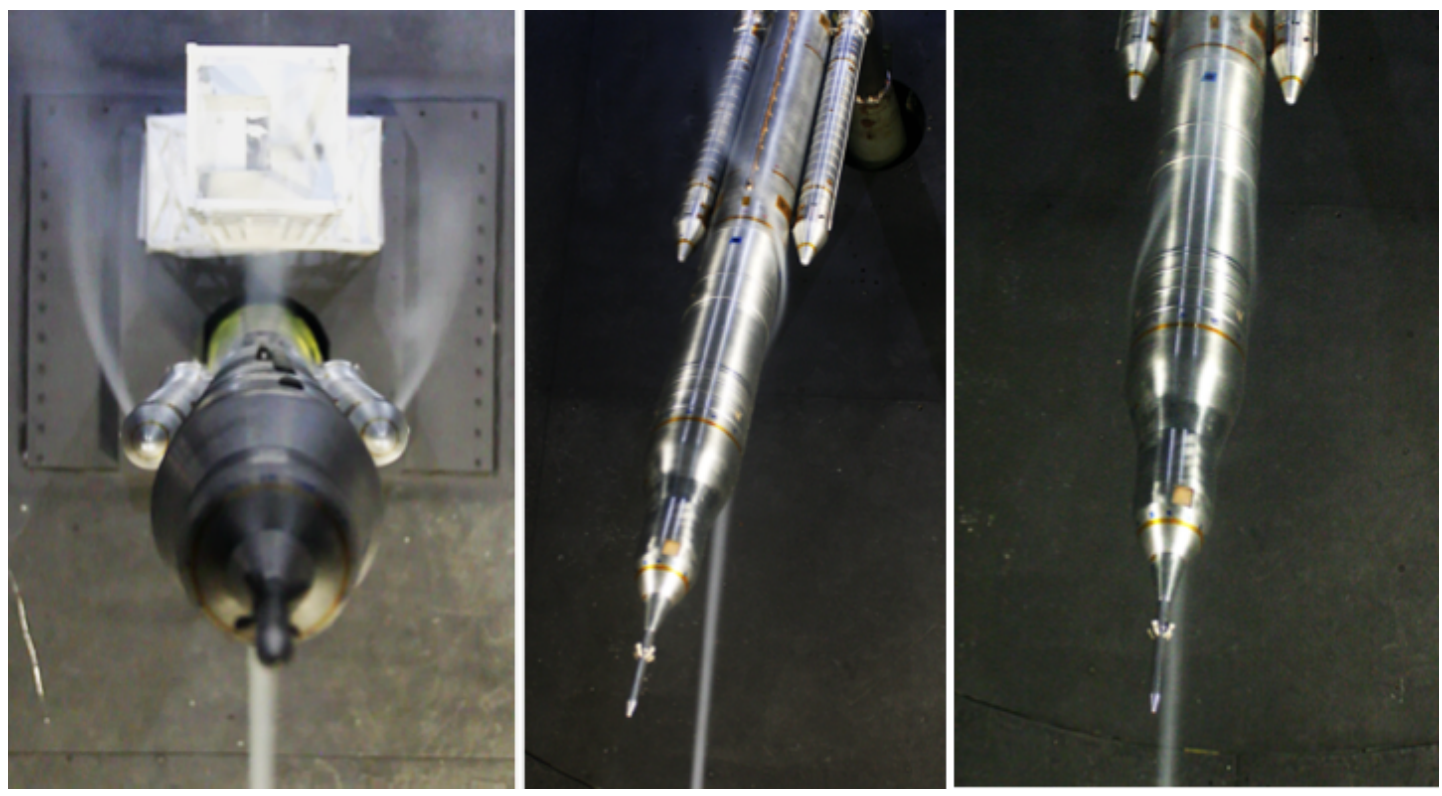

Figure 43. Sample smoke flow visualization photos showing Coandă effect on core body and between core and SRBs. 


\section{Concluding Remarks}

A second low-speed wind tunnel test was recently completed in the NASA Langley Research Center 14- by 22-Foot Subsonic Tunnel to characterize the liftoff and transition aerodynamic environment of the SLS launch vehicle. Test 633 utilized 1.75\%-scale models of three SLS vehicle configurations to acquire aerodynamic forces and moments during the transition phase from liftoff to ascent that covered a wide range of angles of attack and angles of sideslip, and to characterize the tower interference effects as a function of the ground wind azimuth angle and the height of the vehicle above the launch pad. Based on lessons learned from the first SLS LOT test (Test 609), a stiffer sting-balance combination was designed and fabricated to minimize the model dynamics encountered during the test. This allowed data acquisition in areas of the test envelope that were not possible in Test 609, in particular at total angles of attack between $30^{\circ}$ and $60^{\circ}$ and at wind azimuth angles around $30^{\circ}$ and $330^{\circ}$.

Additionally, a new novel force measurement technique using miniature six-component load cells was applied to the SRBs in order to acquire forces and moments for the left and right SRB separately from the full vehicle. The aerodynamic data from the SRBs showed reasonable magnitudes and trends and exhibited symmetry as expected. It also allowed isolation of component loads, which provided insight into the cause of the large asymmetric lateral loading experienced by the core stage at midrange total angles of attack between $30^{\circ}$ and $60^{\circ}$. The tower interference effects on the normal force coefficient were most pronounced for the northerly winds when the vehicle is in the wake of the tower. The westerly winds also showed tower interference effects to the side and normal force coefficients due to a venturi effect between the tower and the vehicle. Finally, smoke flow visualization from the test revealed interesting flow features such as the flow performing an S-turn as it went around the tower, between the tower and vehicle, and finally back around the vehicle at wind azimuth angles around $30^{\circ}$ and $330^{\circ}$.

The test was very successful as all test objectives were achieved, and new test techniques and methods were implemented that solved problems from the first SLS LOT test, while also providing a more complete data set. The overall data set acquired in the test covered the entire parameter space required for conducting flight simulations of the vehicle's liftoff event and were used to update the SLS LOT aerodynamic databases for the SLS Block 1 and Block 1B vehicle configurations.

\section{Acknowledgement}

The authors would like to acknowledge the excellent collaboration between the model designers, force measurement engineers, and the SLS research engineers for making the test campaign a major success. The authors would also like to acknowledge the hard work performed by the $14 \times 22$ facility engineers and technicians throughout the test campaign, and the support by the Space Launch System program offices at the NASA Marshall Space Flight Center and NASA Langley Research Center, as well as the constant support from the SLS Aerodynamics Task Team.

\section{References}

${ }^{1}$ http://www.nasa.gov/sls, "NASA Space Launch System," .

${ }^{2}$ Capone, F. J., Paulson, J. W., and Erickson, G. E., "Liftoff and Transition Aerodynamics of the Ares I Launch Vehicle," AIAA Journal of Spacecraft and Rockets, Vol. 49, No. 4, Jul-Aug 2012, pp. 564-573.

${ }^{3}$ Pinier, J. T., Erickson, G. E., Paulson, J. W., Tomek, W. G., Bennett, D. W., and Blevins, J. A., "Space Launch System Liftoff and Transition Aerodynamic Characterization in the NASA Langley 14- by 22-Foot Subsonic Wind Tunnel," AIAA Paper 2015-0775, AIAA, January 2015.

${ }^{4}$ Gentry, G. L., J., Quinto, P. F., Gatlin, G. M., and Applin, Z. T., "The Langley 14- by 22-Foot Subsonic Tunnel: Description, Flow Characteristics, and Guide for Users," NASA Technical Paper 3008, September 1990.

${ }^{5}$ Parker, P. A., Wind Tunnel Model System Dynamic Analysis and Simulation with Application to Model System Vibration Suppression, Master's thesis, Christopher Newport University, 2000.

${ }^{6}$ Parker, P. A., "Single-Vector Calibration System for Multi-Axis Load Cells and Method for Calibrating a Multi-Axis Load Cell," U.S. Patent 6629446, 2003.

${ }^{7}$ Commo, S. A. and Lynn, K. C., "In-Situ Load System (ILS) for Calibrating and Validating Aerodynamic Properties of Scaled Aircraft in Ground-Based Aerospace Testing Applications," U.S. Patent 9354134, 2016.

${ }^{8}$ Toro, K. G. and Parker, P. A., "Unique Booster Force Measurement on NASA's Space Launch System (SLS)," January 2018, Presented at AIAA SciTech Forum 2018 in Kissimmee, FL.

${ }^{9}$ Commo, S. A., Toro, K. G., Parker, P. A., and Chan, D. T., "Development of an Improved Force Measurement Capa- 
bility for the Liftoff and Transition Wind-Tunnel Test of NASA's Space Launch System," May 2018, Presented at the 11th International Symposium on Strain-Gage Balances in Cologne, Germany.

${ }^{10}$ Krist, S. E., Ratnayake, N. A., and Ghaffari, F., "Kestrel Results at Liftoff Conditions for a Space Launch System Configuration in Proximity to the Launch Tower," AIAA Paper 2019-xxxx, Presented at the 57th AIAA Aerospace Sciences Meeting as part of the SciTech Forum, 2019.

${ }^{11}$ Ratnayake, N. A., Krist, S. E., and Ghaffari, F., "Selection of Computational Fluid Dynamics Tools Used in Development of the Space Launch System Liftoff and Transition Lineloads Databases," AIAA Paper 2019-xxxx, Presented at the 57th AIAA Aerospace Sciences Meeting as part of the SciTech Forum, 2019.

${ }^{12}$ Ericsson, L. E., "Sources of High Alpha Vortex Asymmetry at Zero Sideslip," AIAA Journal of Aircraft, Vol. 29, No. 6, Nov-Dec 1992, pp. 1086-1090.

${ }^{13}$ Kubin, J. S., An Analysis of Steady Asymmetric Vortex Shedding from a Missile at High Angles of Attack, Master's thesis, Air Force Institute of Technology, November 1973. 